\title{
A General Modular Method of Azaindole and Thienopyrrole Synthesis via Pd-Catalyzed Tandem Couplings of Gemdichloroolefins
}

\section{Table of Content}

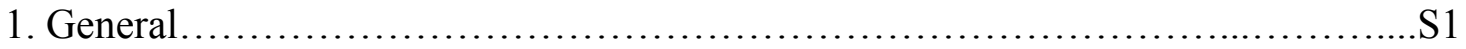



3. Preparation of Azaindole ................................................... 11



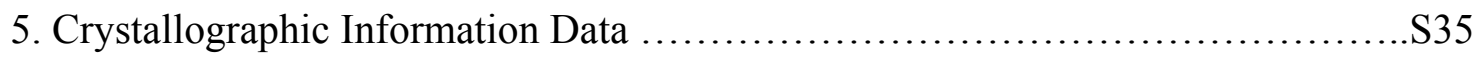

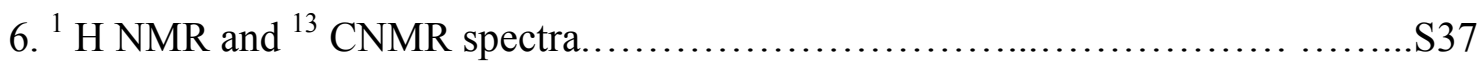

\section{General}

All flasks were flame-dried under a stream of nitrogen or argon and cooled before use unless otherwise noted. Solvents and solutions were transferred with syringes or cannulae using standard inert atmosphere techniques.

${ }^{1} \mathrm{H},{ }^{13} \mathrm{C}$, and ${ }^{19} \mathrm{~F}$ NMR spectra were obtained using a Varian Mercury-300, Gemini300, Unity-400, Mercury 400, or Unity-500 spectrometer. ${ }^{1} \mathrm{H}$ NMR spectra were referenced to tetramethylsilane (TMS, $0 \mathrm{ppm}$ ) using $\mathrm{CDCl}_{3}$ as solvent, $\mathrm{DMSO}-\mathrm{D}_{5}$ residue peaks $\left(2.50 \mathrm{ppm}\right.$ ) using DMSO- $\mathrm{d}_{6}$ as solvent, $\mathrm{CHD}_{2} \mathrm{OD}$ residue peaks $(3.30 \mathrm{ppm})$ using $\mathrm{CD}_{3} \mathrm{OD}$ as a solvent. ${ }^{13} \mathrm{C}$ NMR spectra were referenced to solvent carbons $(77.23 \mathrm{ppm}$ for $\mathrm{CDCl}_{3} ; 39.57 \mathrm{ppm}$ for DMSO-d $\mathrm{d}_{6}, 49.15 \mathrm{ppm}$ for $\mathrm{CD}_{3} \mathrm{OD}$ ). When carbons are equivalent, no special notation is used. ${ }^{19} \mathrm{~F}$ NMR spectra were referenced to $\mathrm{CFCl}_{3}$.

Melting points were taken on a Fisher-Johns melting point apparatus without correction. IR spectra were obtained using a Nicolet DX FT IR spectrometer using thin films of products coated on $\mathrm{NaCl}$ plates. Only absorption frequencies higher than 1000 $\mathrm{cm}^{-1}$ are reported. High-resolution mass spectra were obtained from a VG 70-250S (double focusing) mass spectrometer at $70 \mathrm{eV}$.

Analytical TLC was performed using EM Separations pre-coated silica gel $0.2 \mathrm{~mm}$ layer UV 254 fluorescent sheets. Column chromatography was carried out as "flash chromatography" as reported by Still on Ultra Pure Silica Gel (Silicycles, 230-400 mesh, 
60A) silica gel using the indicated eluent. ${ }^{1}$ Unless otherwise specified, extracts were dried over $\mathrm{MgSO}_{4}$ and volatile solvents were removed with a rotary evaporator at aspirator pressure.

Diethyl ether and toluene were distilled under nitrogen from $\mathrm{Na} /$ benzophenone immediately prior to use. Dichloromethane (DCM) was distilled from $\mathrm{CaH}_{2}$. Dichloromethane and diethyl ether were also purified by passing through a bed of activated molecular sieves using MBraun Solvent Purification System.

\section{Preparation of Substrates}

\section{Benzyl-[3-(2,2-dibromovinyl)-pyridin-2-yl]amine (5)}
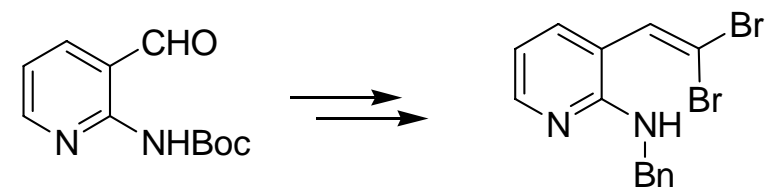

The procedure for the preparation of $\mathbf{1 8}$ was followed using the aldehyde $(0.336 \mathrm{~g}$, $1.5 \mathrm{mmol}), \mathrm{BnBr}(0.22 \mathrm{~mL}, 1.87 \mathrm{mmol})$, and $\mathrm{NaH}(0.082 \mathrm{~g}, 60 \%, 2 \mathrm{mmol})$. The benzylated intermediate was obtained as an oil $(0.350 \mathrm{~g}, 75 \%)$. The procedure for the second step was also followed using the benzylated amide $(0.350 \mathrm{~g}, 1.12 \mathrm{mmol}), \mathrm{CBr}_{4}$ $(0.570 \mathrm{~g}, 1.68 \mathrm{mmol})$, and $\mathrm{PPh}_{3}(0.881 \mathrm{~g}, 3.36 \mathrm{mmol})$. The product 5 was obtained as a slightly yellow oil ( $0.197 \mathrm{~g}, 48 \%$ over 2 steps) by flash chromatography using $20 \%$ EtOAc in hexanes. IR (neat, $\left.\mathrm{cm}^{-1}\right): 3424,3040,1583,1498 .{ }^{1} \mathrm{H}$ NMR $\left(400 \mathrm{MHz}, \mathrm{CDCl}_{3}\right.$ ) $\delta 8.17(1 \mathrm{H}, \mathrm{dd}, J=4.8,1.8 \mathrm{~Hz}), 7.54(1 \mathrm{H}, \mathrm{ddd}, J=7.5,1.8,0.9 \mathrm{~Hz}), 7.44-7.28(5 \mathrm{H}, \mathrm{m})$, $7.18(1 \mathrm{H}, \mathrm{s}), 6.68(1 \mathrm{H}, \mathrm{dd}, J=7.5,5.1 \mathrm{~Hz}), 4.70(2 \mathrm{H}, \mathrm{d}, J=5.5 \mathrm{~Hz}), 4.52(1 \mathrm{H}, \mathrm{br}) .{ }^{13} \mathrm{C}$ NMR (100 MHz, $\left.\mathrm{CDCl}_{3}\right) \delta 154.9,148.6,139.7,137.2,132.4,128.9,128.1,127.5,116.6$, 112.9, 95.0, 45.9. HRMS (EI) calc'd for $\mathrm{C}_{14} \mathrm{H}_{12} \mathrm{~N}_{2} \mathrm{Br}_{2}\left([\mathrm{M}]^{+}\right)$365.9367. Found: 365.9367 .

\section{[3-(2,2-dichloro-1-methylvinyl)-pyridin-2-yl]-carbamic acid tert-butyl ester (20)}

\footnotetext{
${ }^{1}$ Still, W. C.; Kahn, M.; Mitra, A. J. Org. Chem. 1978, 43, 2923.
} 
<smiles>[Pb]=Nc1ccccn1</smiles>

<smiles>CC(=C(Cl)Cl)c1cccnc1NP</smiles>

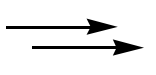<smiles>CC(=O)Nc1ncccc1C(C)=C(Cl)Cl</smiles>

To a solution of 2-PivNH pyridine $(2.82 \mathrm{~g}, 16 \mathrm{mmol})$ in THF $(40 \mathrm{~mL})$ was added $n$ BuLi dropwise at $-78^{\circ} \mathrm{C}$. After addition, the mixture was warmed to $0{ }^{\circ} \mathrm{C}$ in an ice bath and stirred for $2 \mathrm{~h}$. The mixture was cooled to $-78{ }^{\circ} \mathrm{C}$, and a solution of $N$-methoxy- $N$ methylacetamide $(2.0 \mathrm{~g}, 19 \mathrm{mmol})$ in THF $(10 \mathrm{~mL})$ was added. The mixture was stirred for 10 min and warmed to rt overnight. The reaction was quenched by addition of $\mathrm{NH}_{4} \mathrm{Cl}$ $(30 \mathrm{~mL})$, extracted with $\mathrm{Et}_{2} \mathrm{O}(30 \mathrm{~mL})$, washed by $\mathrm{NaHCO}_{3}$, brine, and dried over $\mathrm{Na}_{2} \mathrm{SO}_{4}$. The crude material was purified by flash chromatography $(50 \% \rightarrow 100 \%$ EtOAc in hexanes) to afford a white solid $(1.75 \mathrm{~g}, 50 \%) . \mathrm{mp} 67-68{ }^{\circ} \mathrm{C}$. IR (neat, $\mathrm{cm}^{-1}$ ): 3268 , 2967, 1710, 1694, 1663, 1580, 1504, 1450, 1263, 1153. ${ }^{1} \mathrm{H}$ NMR $\left(300 \mathrm{MHz}, \mathrm{CDCl}_{3}\right) \delta$ $11.53(1 \mathrm{H}, \mathrm{s}), 8.66(1 \mathrm{H}, \mathrm{dd}, J=4.7,1.8 \mathrm{~Hz}), 8.19(1 \mathrm{H}, \mathrm{dd}, J=7.9,2.0 \mathrm{~Hz}), 7.10(1 \mathrm{H}, \mathrm{dd}$, $J=7.9,4.8 \mathrm{~Hz}), 2.67(3 \mathrm{H}, \mathrm{s}), 1.37(9 \mathrm{H}, \mathrm{s}) .{ }^{13} \mathrm{C} \mathrm{NMR}\left(100 \mathrm{MHz}, \mathrm{CDCl}_{3}\right) \delta 201.1,176.9$, 153.7, 152.2, 140.0, 118.1, 40.7, 28.1, 27.5. HRMS (EI) calc'd for $\mathrm{C}_{12} \mathrm{H}_{16} \mathrm{~N}_{2} \mathrm{O}_{2}\left([\mathrm{M}]^{+}\right)$ 220.1211. Found: 220.1211.

The general procedure for the preparation of gemdichlorovinyl substrates was followed using the ketone intermediate $(1.10 \mathrm{~g}, 5 \mathrm{mmol}), \mathrm{KOtBu} \cdot \mathrm{HOtBu}(1.86 \mathrm{~g}, 10$ mmol), powdered $\mathrm{PPh}_{3}(2.62 \mathrm{~g}, 10 \mathrm{mmol})$, and $\mathrm{CHCl}_{3}(1.19 \mathrm{~g}, 10 \mathrm{mmol})$. The product 12 was obtained as a white solid (1.276 g, 89\%). mp 116-118 ${ }^{\circ} \mathrm{C}$. IR (neat, $\mathrm{cm}^{-1}$ ): 3210, 2967, 1680, 1516, 1438, 1285, 1166. ${ }^{1} \mathrm{H}$ NMR (400 MHz, $\left.\mathrm{CDCl}_{3}\right) \delta 8.38(1 \mathrm{H}, \mathrm{dd}, J=$ 4.8, $1.7 \mathrm{~Hz}), 7.91(1 \mathrm{H}, \mathrm{br}), 7.55(1 \mathrm{H}, \mathrm{dd}, J=7.7,1.8 \mathrm{~Hz}), 7.19(1 \mathrm{H}, \mathrm{dd}, J=7.8,4.8 \mathrm{~Hz})$, $2.25(3 \mathrm{H}, \mathrm{s}), 1.32(9 \mathrm{H}, \mathrm{s}) .{ }^{13} \mathrm{C} \mathrm{NMR}\left(100 \mathrm{MHz}, \mathrm{CDCl}_{3}\right) \delta 177.01,148.2,147.6,137.7$, 133.2, 130.7, 121.4, 117.6, 39.7, 27.7, 21.8. HRMS (EI) calc'd for $\mathrm{C}_{13} \mathrm{H}_{16} \mathrm{~N}_{2} \mathrm{OCl}_{2}\left([\mathrm{M}]^{+}\right)$ 286.0640. Found: 286.0643.

A suspension of $12(1.265 \mathrm{~g}, 4.4 \mathrm{mmol})$ in $\mathrm{HCl}(6 \mathrm{M}, 10 \mathrm{~mL})$ was heated at $110{ }^{\circ} \mathrm{C}$ overnight $(12 \mathrm{~h})$. The mixture was diluted with $\mathrm{H}_{2} \mathrm{O}(10 \mathrm{~mL})$, neutralized with solid $\mathrm{K}_{2} \mathrm{CO}_{3}$, extracted with $\mathrm{Et}_{2} \mathrm{O}(3 \times 20 \mathrm{~mL})$, washed with brine, and dried over $\mathrm{Na}_{2} \mathrm{SO}_{4}$. The crude material was purified using flash chromatography $(33 \% \rightarrow 50 \%$ EtOAc in hexanes) to afford a white solid $(0.836 \mathrm{~g}, 94 \%)$. mp $82-83{ }^{\circ} \mathrm{C}$. IR (neat, $\mathrm{cm}^{-1}$ ): $3474,3297,3162$, 
1631, 1574, 1454. ${ }^{1} \mathrm{H}$ NMR $\left(400 \mathrm{MHz}, \mathrm{CDCl}_{3}\right) \delta 8.06(1 \mathrm{H}, \mathrm{dd}, J=4.6,1.5 \mathrm{~Hz}), 7.24$ $(1 \mathrm{H}, \mathrm{dd}, J=7.5,1.9 \mathrm{~Hz}), 6.70$ (1H, ddd, $J=7.5,5.0,0.5 \mathrm{~Hz}), 4.44(2 \mathrm{H}, \mathrm{br}), 2.14(3 \mathrm{H}, \mathrm{s})$. ${ }^{13} \mathrm{C}$ NMR $\left(100 \mathrm{MHz}, \mathrm{CDCl}_{3}\right) \delta 154.6,148.2,136.8,132.4,120.2,119.7$ 114.4, 21.3. HRMS (EI) calc'd for $\mathrm{C}_{8} \mathrm{H}_{8} \mathrm{~N}_{2} \mathrm{Cl}_{2}$ ([M] $]^{+}$) 202.0065. Found: 202.0063.

A mixture of the pyridine amine $(0.102 \mathrm{mg}, 0.5 \mathrm{mmol})$ and $\mathrm{Boc}_{2} \mathrm{O}(0.120 \mathrm{mmol}, 0.55$ mmol) in $t-\mathrm{BuOH}(2 \mathrm{~mL})$ was stirred at $\mathrm{rt}$ for $38 \mathrm{~h}$ to result a white suspension. The solvent was removed under vacuum and the residue was added into a $\mathrm{NaHCO}_{3} / \mathrm{K}_{2} \mathrm{CO}_{3}$ solution. The mixture was extracted with EtOAc $(25 \mathrm{~mL})$, washed with brine, and dried over $\mathrm{Na}_{2} \mathrm{SO}_{4}$. The crude material was purified using flash chromatography $(25 \%$ EtOAc in hexanes) to afford product 20 as a white solid $(0.110 \mathrm{~g}, 72 \%)$. mp $172-174{ }^{\circ} \mathrm{C}$. IR (neat, $\left.\mathrm{cm}^{-1}\right): 3162,2974,1725,1519,1448,1271,1161 .{ }^{1} \mathrm{H}$ NMR $\left(400 \mathrm{MHz}, \mathrm{CDCl}_{3}\right) \delta$ $8.41(1 \mathrm{H}, \mathrm{dd}, J=4.8,1.8 \mathrm{~Hz}), 7.49(1 \mathrm{H}, \mathrm{dd}, J=7.7,1.8 \mathrm{~Hz}), 7.09(1 \mathrm{H}, \mathrm{dd}, J=7.5,4.8$ $\mathrm{Hz}), 7.03(1 \mathrm{H}, \mathrm{br}), 2.20(3 \mathrm{H}, \mathrm{s}), 1.52(9 \mathrm{H}, \mathrm{s}) .{ }^{13} \mathrm{C} \mathrm{NMR}\left(100 \mathrm{MHz}, \mathrm{CDCl}_{3}\right) \delta 152.2$, 148.4, 147.8, 137.9, 132.3, 127.6, 120.0, 119.3, 81.3, 28.4, 21.8. HRMS (ESI) calc'd for $\mathrm{C}_{13} \mathrm{H}_{17} \mathrm{~N}_{2} \mathrm{O}_{2} \mathrm{Cl}_{2}\left([\mathrm{M}+\mathrm{H}]^{+}\right)$303.0667. Found: 303.0660 .

\section{[4-(2,2-dichlorovinyl)pyridin-3-yl]-carbamic acid tert-butyl ester (14)}

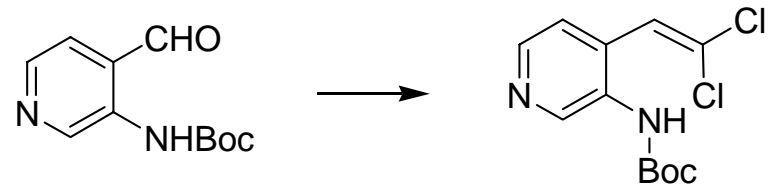

The general procedure for the preparation of gemdichlorovinyl substrates was followed using 3-NHBoc-4-pyridinecarboxaldehyde (1.11 g, $5 \mathrm{mmol}), \mathrm{KOtBu} \cdot \mathrm{HOtBu}$ (1.40 g, $7.5 \mathrm{mmol}$ ), powdered $\mathrm{PPh}_{3}(1.97 \mathrm{~g}, 7.5 \mathrm{mmol})$, and $\mathrm{CHCl}_{3}(0.89 \mathrm{~g}, 7.5 \mathrm{mmol})$. Substrate 14 was obtained as a white solid $(0.87 \mathrm{~g}, 60 \%)$. mp 120-122 ${ }^{\circ} \mathrm{C}$. IR (neat, $\left.\mathrm{cm}^{-1}\right)$ : 3221, 2977, 1728, 1556, 1515, 1418, 1247, 1160. ${ }^{1} \mathrm{H}$ NMR (400 MHz, $\left.\mathrm{CDCl}_{3}\right) \delta 9.02$ (1H, br), $8.39(1 \mathrm{H}, \mathrm{d}, J=5.1 \mathrm{~Hz}), 7.37(1 \mathrm{H}, \mathrm{d}, J=5.1 \mathrm{~Hz}), 6.78(1 \mathrm{H}, \mathrm{s}), 6.22(1 \mathrm{H}, \mathrm{br})$, $1.54(9 \mathrm{H}, \mathrm{s}) .{ }^{13} \mathrm{C} \mathrm{NMR}\left(100 \mathrm{MHz}, \mathrm{CDCl}_{3}\right) \delta 152.8,145.2,144.9,132.9,132.1,127.4$, 123.0, 122.9, 81.9, 28.4. HRMS (ESI) calc'd for $\mathrm{C}_{12} \mathrm{H}_{15} \mathrm{~N}_{2} \mathrm{O}_{2} \mathrm{Cl}_{2}\left([\mathrm{M}+\mathrm{H}]^{+}\right) 289.0505$. Found: 289.0514. 
[4-(2,2-dichlorovinyl)pyridin-3-yl]-carbamic acid tert-butyl ester (10)

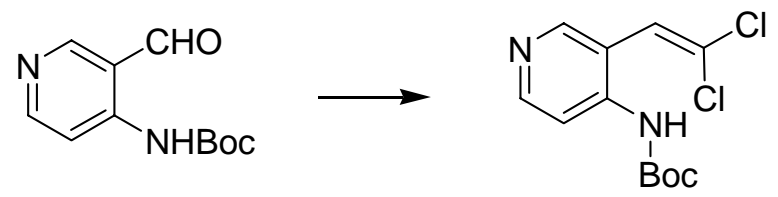

The general procedure for the preparation of gemdichlorovinyl substrates was followed using 4-NHBoc-3-pyridinecarboxaldehyde (5.56 g, $25 \mathrm{mmol}), \mathrm{KO} \mathrm{Bu} \cdot \mathrm{HOtBu}$ (6.98 g, $37.5 \mathrm{mmol}$ ), powdered $\mathrm{PPh}_{3}(9.83 \mathrm{~g}, 37.5 \mathrm{mmol})$, and $\mathrm{CHCl}_{3}(4.46 \mathrm{~g}, 37.5$ mmol). Substrate 10 was obtained as a white solid (6.82 g, 94\%). mp $148-149{ }^{\circ} \mathrm{C}$. IR (neat, $\mathrm{cm}^{-1}$ ): 3196, 2974, 1733, 1574, 1511, 1245, 1154. ${ }^{1} \mathrm{H}$ NMR $\left(400 \mathrm{MHz}, \mathrm{CDCl}_{3}\right) \delta$ $8.46(1 \mathrm{H}, \mathrm{s}), 8.45(1 \mathrm{H}, \mathrm{d}, J=5.7 \mathrm{~Hz}), 8.06,(1 \mathrm{H}, \mathrm{d}, J=5.7 \mathrm{~Hz}), 6.71(1 \mathrm{H}, \mathrm{s}), 6.50(1 \mathrm{H}$, br), $1.55(9 \mathrm{H}, \mathrm{s}) .{ }^{13} \mathrm{C}$ NMR $\left(100 \mathrm{MHz}, \mathrm{CDCl}_{3}\right) \delta 151.8,150.7,150.4,143.3,127.7,121.5$, 118.2, 112.8, 82.4, 28.4. HRMS (ESI) calc'd for $\mathrm{C}_{12} \mathrm{H}_{15} \mathrm{~N}_{2} \mathrm{O}_{2} \mathrm{Cl}_{2}\left([\mathrm{M}+\mathrm{H}]^{+}\right) 289.0505$. Found: 289.0512.

\section{(2-Formylthiophen-3-yl)carbamic acid tert-butyl ester}
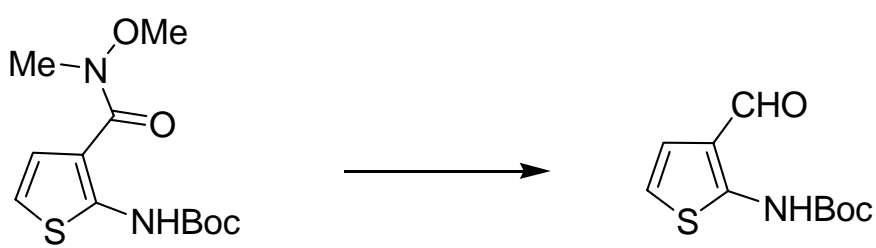

To a solution of the Weinreb amide $(0.286 \mathrm{~g}, 1 \mathrm{mmol})$ in THF $(5 \mathrm{~mL})$ was added dropwise DIBAL-H solution ( $1 \mathrm{M}$ in pentane, $1 \mathrm{~mL}, 1 \mathrm{mmol}$ ) at $-78^{\circ} \mathrm{C}$. The mixture was warmed to $-30{ }^{\circ} \mathrm{C}$, and stirred for $1 \mathrm{~h}$, and warmed to $0{ }^{\circ} \mathrm{C}$, quenched by $\mathrm{MeOH}(0.5 \mathrm{~mL})$. To the mixture was added a saturated solution of Rochelle's salt. The resulting mixture was extracted with $\mathrm{Et}_{2} \mathrm{O}(3 \times 15 \mathrm{~mL})$, and the combined organic layers were dried over $\mathrm{MgSO}_{4}$. The crude material was purified using flash chromatography using 5\% EtOAc in hexanes to afford the aldehyde as an oil. ${ }^{1} \mathrm{H}$ NMR $\left(400 \mathrm{MHz}, \mathrm{CDCl}_{3}\right) \delta 10.51(1 \mathrm{H}, \mathrm{br})$, $9.80(1 \mathrm{H}, \mathrm{s}), 7.11(1 \mathrm{H}, \mathrm{d}, J=5.9 \mathrm{~Hz}), 6.68(1 \mathrm{H}, \mathrm{dd}, J=5.7,0.9 \mathrm{~Hz}), 1.55(9 \mathrm{H}, \mathrm{s}) .{ }^{13} \mathrm{C}$ NMR (100 MHz, $\left.\mathrm{CDCl}_{3}\right) \delta 186.4,152.4,125.3,120.9,115.5,82.8,28.1$. 


\section{[3-(2,2-Dichlorovinyl)thiophen-2-yl]-carbamic acid tert-butyl ester (16)}

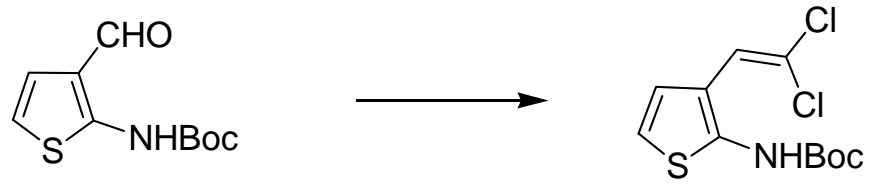

The general procedure for the preparation of gemdichlorovinyl substrates was followed using 2-NHBoc-3-thiophenecarboxaldehyde (2.28 g, $10 \mathrm{mmol}), \mathrm{KO} \mathrm{Bu} \cdot \mathrm{HO}$ Bu (3.72 g, $20 \mathrm{mmol}$ ), powdered $\mathrm{PPh}_{3}(5.24 \mathrm{~g}, 20 \mathrm{mmol})$, and $\mathrm{CHCl}_{3}(2.38 \mathrm{~g}, 20 \mathrm{mmol})$. Substrate 16 was obtained as a white solid (2.23 g, 76\%). mp 120-121 ${ }^{\circ} \mathrm{C}$. IR (neat, $\mathrm{cm}^{-1}$ ): 3200, 2980, 1682, 1553, 1501, 1275, 1153. ${ }^{1} \mathrm{H}$ NMR (400 MHz, $\left.\mathrm{CDCl}_{3}\right) \delta 7.22(1 \mathrm{H}, \mathrm{d}, J$ $=5.7 \mathrm{~Hz}), 6.88(1 \mathrm{H}, \mathrm{d}, J=5.7 \mathrm{~Hz}), 6.78(1 \mathrm{H}, \mathrm{br}), 6.66(1 \mathrm{H}, \mathrm{s}), 1.53(9 \mathrm{H}, \mathrm{s}) .{ }^{13} \mathrm{C} \mathrm{NMR}$ $\left(100 \mathrm{MHz}, \mathrm{CDCl}_{3}\right) \delta 152.5,138.5,124.5,121.5,120.8,118.8,117.8,82.4,28.4$. HRMS (EI) calc'd for $\mathrm{C}_{11} \mathrm{H}_{13} \mathrm{NO}_{2} \mathrm{SCl}_{2}\left([\mathrm{M}]^{+}\right)$293.0044. Found: 293.0039 .

\section{(2-Formylthiophen-3-yl)carbamic acid tert-butyl ester}

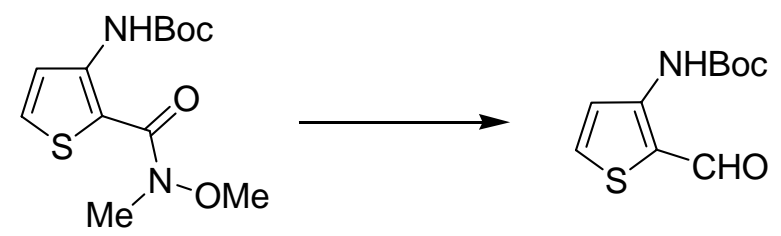

To a solution of the Weinreb amide $(0.450 \mathrm{~g}, 1.57 \mathrm{mmol})$ in $\mathrm{Et}_{2} \mathrm{O}(10 \mathrm{~mL})$ at $0{ }^{\circ} \mathrm{C}$ was added $\mathrm{LiAlH}_{4}(72 \mathrm{mg}, 1.89 \mathrm{mmol})$ in one portion. The suspension was stirred for $40 \mathrm{~min}$ and warmed to rt. The mixture was quenched by addition of a saturated solution of Rochelle's salt, extracted with $\mathrm{Et}_{2} \mathrm{O}(3 \times 15 \mathrm{~mL})$, dried over $\mathrm{MgSO}_{4}$. The crude material was purified by flash chromatography using 5\% EtOAc in hexanes to afford the aldehyde as a colorless oil $(0.260 \mathrm{~g}, 73 \%) .{ }^{1} \mathrm{H}$ NMR $\left(500 \mathrm{MHz}, \mathrm{CDCl}_{3}\right) \delta 9.81(1 \mathrm{H}, \mathrm{br}), 9.67(1 \mathrm{H}$, s), $7.94(1 \mathrm{H}, \mathrm{d}, J=5.1 \mathrm{~Hz}), 7.66(1 \mathrm{H}, \mathrm{d}, J=5.4 \mathrm{~Hz}), 1.53(9 \mathrm{H}, \mathrm{s}) .{ }^{13} \mathrm{C} \mathrm{NMR}(100 \mathrm{MHz}$, $\left.\mathrm{CDCl}_{3}\right) \delta 183.8,152.3,145.8,136.2,122.1,119.6,81.8,28.4$.

\section{[2-(2,2-Dichlorovinyl)thiophen-3-yl]carbamic acid tert-butyl ester (17)}




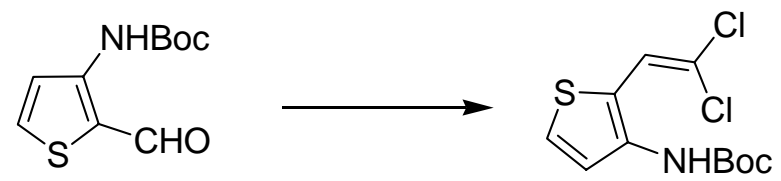

The general procedure for the preparation of gemdichlorovinyl substrates was followed using 3-NHBoc-2-thiophenecarboxaldehyde (1.00 g, $4.4 \mathrm{mmol}), \mathrm{KOtBu} \cdot \mathrm{HO} t \mathrm{Bu}$ (1.86 g, $10 \mathrm{mmol})$, powdered $\mathrm{PPh}_{3}(2.62 \mathrm{~g}, 10 \mathrm{mmol})$, and $\mathrm{CHCl}_{3}(1.19 \mathrm{~g}, 10 \mathrm{mmol})$. Substrate 17 was obtained as a white solid $(1.03 \mathrm{~g}, 80 \%)$. mp 132-133 ${ }^{\circ} \mathrm{C}$. IR (neat, $\left.\mathrm{cm}^{-1}\right)$ : 3268, 2978, 1691, 1553, 1367, 1248, 1159. ${ }^{1} \mathrm{H}$ NMR (400 MHz, $\left.\mathrm{CDCl}_{3}\right) \delta$ 7.34-7.30 (2H, m, br), $6.89\left(1 \mathrm{H}\right.$, br), $6.43(1 \mathrm{H}, \mathrm{br}), 1.52(9 \mathrm{H}, \mathrm{s}) .{ }^{13} \mathrm{C} \mathrm{NMR}\left(100 \mathrm{MHz}, \mathrm{CDCl}_{3}\right) \delta 153.0$, 135.8, 126.1, 123.6, 119.6, 119.2, 81.5, 28.5. HRMS (EI) calc'd for $\mathrm{C}_{11} \mathrm{H}_{13} \mathrm{NO}_{2} \mathrm{SCl}_{2}$ $\left([\mathrm{M}]^{+}\right)$293.0044. Found: 293.0039.

\section{[3-(2,2-Dichlorovinyl)-pyridin-2-yl]-methyl-amine (19)}



To a suspension of $11(0.289 \mathrm{~g}, 1 \mathrm{mmol})$ in DMF $(3 \mathrm{~mL})$ was added MeI ( $84 \mu \mathrm{L}, 1.35$ $\mathrm{mmol})$ at $0{ }^{\circ} \mathrm{C}$. To the mixture was added $\mathrm{NaH}(0.052 \mathrm{~g}, 60 \%$ mineral oil, $1.35 \mathrm{mmol})$ in three portions over $15 \mathrm{~min}$, and stirred for an additional $15 \mathrm{~min}$. The mixture was warmed to $\mathrm{rt}$, and stirred for $30 \mathrm{~min}$ before quenched with $\mathrm{NaHCO}_{3}(10 \mathrm{~mL})$. The mixture was extracted with $\mathrm{Et}_{2} \mathrm{O}(3 \times 10 \mathrm{~mL})$, and the combined organic layers were washed with $\mathrm{H}_{2} \mathrm{O}$ $(10 \mathrm{~mL}), \mathrm{NaHCO}_{3}(10 \mathrm{~mL})$, brine $(10 \mathrm{~mL})$, and dried over $\mathrm{Na}_{2} \mathrm{SO}_{4}$. The crude material was further purified by flash chromatography $(15-20 \%$ EtOAc in hexanes) to afford the methylated amide as an oil (0.296 g, 98\%), which was used directly in the next step. The oil was added into an aqueous $\mathrm{HCl}$ solution $(10 \mathrm{~mL}, 3 \mathrm{M})$, and heated at $75^{\circ} \mathrm{C}$ for $2 \mathrm{~h}$. The mixture was then basified using $\mathrm{K}_{2} \mathrm{CO}_{3}$, extracted with $\mathrm{Et}_{2} \mathrm{O}(3 \times 15 \mathrm{~mL})$, and dried over $\mathrm{Na}_{2} \mathrm{SO}_{4}$. The product was purified by flash chromatography $(20 \%$ EtOAc in hexanes) to afford 19 as a white solid (0.1704 g, 84\% over 2 steps). mp $76-7{ }^{\circ} \mathrm{C}$. IR (neat, $\mathrm{cm}^{-1}$ ): 3340, 3271, 2940, 1593, 1574, 1395, 1276. ${ }^{1} \mathrm{H}$ NMR (400 $\left.\mathrm{MHz}, \mathrm{CDCl}_{3}\right) \delta$ $8.15(1 \mathrm{H}, \mathrm{dd}, J=5.1,1.8 \mathrm{~Hz}), 7.51(1 \mathrm{H}, \mathrm{d}, J=7.5 \mathrm{~Hz}), 6.62(1 \mathrm{H}, \mathrm{dd}, J=7.5,5.1 \mathrm{~Hz})$, 
$6.58(1 \mathrm{H}, \mathrm{s}), 4.29(2 \mathrm{H}, \mathrm{br}), 3.03(3 \mathrm{H}, \mathrm{d}, J=4.8 \mathrm{~Hz}) .{ }^{13} \mathrm{C} \mathrm{NMR}\left(100 \mathrm{MHz}, \mathrm{CDCl}_{3}\right) \delta$ 156.1, 148.5, 137.0, 125.2, 123.7, 114.3, 112.4, 28.9. HRMS (ESI) calc'd for $\mathrm{C}_{8} \mathrm{H}_{9} \mathrm{~N}_{2} \mathrm{Cl}_{2}$ $\left([\mathrm{M}+\mathrm{H}]^{+}\right)$203.0137. Found: 203.0131.

\section{Benzyl-[3-(2,2-dichlorovinyl)-pyridin-2-yl]-amine (7)}
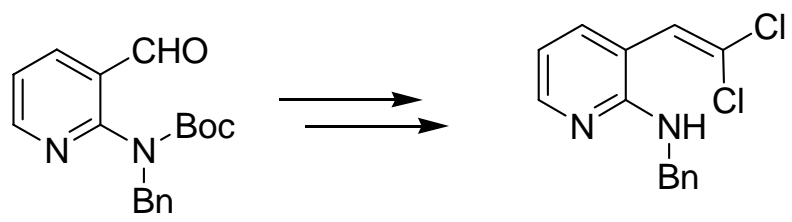

The same procedure for benzylation of 2-BocNH-3-pyridinecarboxaldehyde (first step for the preparation of 18) was used for obtaining 2-Boc(Bn)-3pyridinecarboxaldehyde. The general procedure for the preparation of gemdichlorovinyl substrates was followed using the aldehyde $(0.275 \mathrm{~g}, 0.88 \mathrm{mmol}), \mathrm{KO} t \mathrm{Bu} \cdot \mathrm{HO} t \mathrm{Bu}(0.327$ $\mathrm{g}, 1.76 \mathrm{mmol})$, powdered $\mathrm{PPh}_{3}(0.461 \mathrm{~g}, 1.76 \mathrm{mmol})$, and $\mathrm{CHCl}_{3}(0.209 \mathrm{~g}, 1.76 \mathrm{mmol})$. The BocNH intermediate was obtained as a colorless oil, which was added into an aqueous $\mathrm{HCl}$ solution $(10 \mathrm{~mL}, 3 \mathrm{M})$, and heated at $75^{\circ} \mathrm{C}$ for $2 \mathrm{~h}$. The mixture was then basified using $\mathrm{K}_{2} \mathrm{CO}_{3}$, extracted with $\mathrm{Et}_{2} \mathrm{O}(3 \times 15 \mathrm{~mL})$, and dried over $\mathrm{Na}_{2} \mathrm{SO}_{4}$. The crude material was purified by flash chromatography (15\% EtOAc in hexanes) to afford a yellowish solid ( $0.227 \mathrm{~g}, 93 \%$ in 2 steps). $\mathrm{mp} 57-58{ }^{\circ} \mathrm{C}$. IR (neat, $\left.\mathrm{cm}^{-1}\right): 3438,3316$, 3027, 1588, 1498, 1407, 1274. ${ }^{1} \mathrm{H}$ NMR $\left(400 \mathrm{MHz}, \mathrm{CDCl}_{3}\right) \delta 8.14(1 \mathrm{H}, \mathrm{dd}, J=4.8,1.5$ $\mathrm{Hz}), 7.55(1 \mathrm{H}, \mathrm{d}, J=6.8 \mathrm{~Hz}), 7.38-7.27(5 \mathrm{H}, \mathrm{m}), 6.65(1 \mathrm{H}, \mathrm{dd}, J=7.2,5.1 \mathrm{~Hz}), 6.58(1 \mathrm{H}$, s), $4.67(2 \mathrm{H}, \mathrm{d}, J=5.5 \mathrm{~Hz}), 4.49(1 \mathrm{H}, \mathrm{br}) .{ }^{13} \mathrm{C} \mathrm{NMR}\left(100 \mathrm{MHz}, \mathrm{CDCl}_{3}\right) \delta 155.2,148.5$, 139.7, 137.3, 128.8, 128.1, 127.5, 125.3, 123.5, 114.1, 112.9, 45.9. HRMS (ESI) calc'd for $\mathrm{C}_{14} \mathrm{H}_{13} \mathrm{~N}_{2} \mathrm{Cl}_{2}\left([\mathrm{M}+\mathrm{H}]^{+}\right)$279.0450. Found: 279.0456.

\section{2-(2,2-dichlorovinyl)-pyridin-3-ylamine (15)}



The general procedure for the preparation of gemdichlorovinyl substrate was followed using (5.15 g, $25 \mathrm{mmol}), \mathrm{KOtBu} \cdot \mathrm{HOtBu}(9.3 \mathrm{~g}, 50 \mathrm{mmol})$, powdered $\mathrm{PPh}_{3}(13.1 \mathrm{~g}, 50$ 
mmol), and $\mathrm{CHCl}_{3}(5.95 \mathrm{~g}, 20 \mathrm{mmol})$. The PivNH intermediate was obtained with minor contamination of $\mathrm{PPh}_{3}(\mathrm{O})$. The mixture was added in $\mathrm{HCl}(6 \mathrm{M}, 50 \mathrm{~mL})$, and heated at $110^{\circ} \mathrm{C}$ overnight $(12 \mathrm{~h})$. The mixture was cooled to $\mathrm{rt}$, extracted with $\mathrm{Et}_{2} \mathrm{O}(3 \times 20 \mathrm{~mL})$. The aqueous layer was neutralized with $\mathrm{NaOH}$ and $\mathrm{K}_{2} \mathrm{CO}_{3}$, extracted with EtOAc $(3 \times 50$ $\mathrm{mL}$ ), washed with brine, and dried over $\mathrm{Na}_{2} \mathrm{SO}_{4}$. The crude material was further purified by flash chromatography to afford the product was a white crystalline solid $(3.45 \mathrm{~g}, 73 \%$ over 2 steps). mp 79-80 ${ }^{\circ} \mathrm{C}$. IR (neat, $\mathrm{cm}^{-1}$ ): 3332, 3206, 1613, 1582, 1449, 1313, 1264. ${ }^{1} \mathrm{H}$ NMR $\left(400 \mathrm{MHz}, \mathrm{CDCl}_{3}\right) \delta 8.09\left(1 \mathrm{H}, \mathrm{ABX}, J_{\mathrm{AX}}=4.5 \mathrm{~Hz}, J_{\mathrm{BX}}=1.7 \mathrm{~Hz}\right), 7.07(1 \mathrm{H}$, $\left.\mathrm{ABX}, J_{\mathrm{AB}}=8.2 \mathrm{~Hz}, J_{\mathrm{AX}}=4.5 \mathrm{~Hz}\right), 7.02\left(1 \mathrm{H}, \mathrm{ABX}, J_{\mathrm{AB}}=8.2 \mathrm{~Hz}, J_{\mathrm{BX}}=1.7 \mathrm{~Hz}\right), 6.87$ $(1 \mathrm{H}, \mathrm{s}), 3.81(2 \mathrm{H}, \mathrm{br}) .{ }^{13} \mathrm{C} \mathrm{NMR}\left(100 \mathrm{MHz}, \mathrm{CDCl}_{3}\right) \delta 140.7,140.0,138.4,126.5,124.9$, 124.2, 123.0. HRMS (ESI) calc'd for $\mathrm{C}_{7} \mathrm{H}_{6} \mathrm{~N}_{2} \mathrm{Cl}_{2}\left([\mathrm{M}+\mathrm{H}]^{+}\right)$188.9980. Found: 188.9983.

\section{[2-(2,2-dichlorovinyl)-1-oxypyridin-3-yl]-carbamic acid benzyl ester (34)}

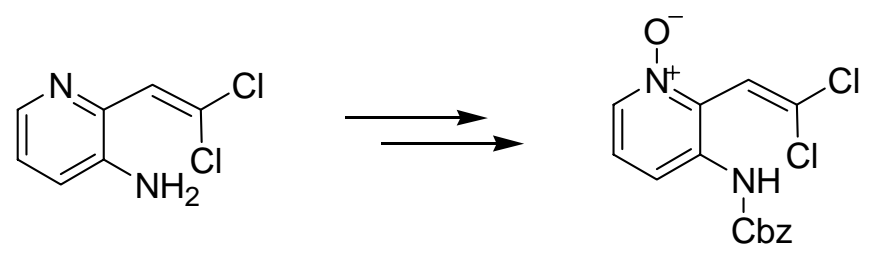

Benzyl chloroformate $(0.68 \mathrm{~mL}, 4.8 \mathrm{mmol})$ was added to a suspension of $15(0.600 \mathrm{~g}$, $3.2 \mathrm{mmol})$ in saturated aqueous $\mathrm{NaHCO}_{3}$ solution $(6 \mathrm{~mL})$ and acetone $(24 \mathrm{~mL})$ at $0{ }^{\circ} \mathrm{C}$. The reaction was warmed slowly to $\mathrm{rt}$, and stirred for $7 \mathrm{~h}$ then re-cooled to $0{ }^{\circ} \mathrm{C}$, and more benzyl chloroformate $(1.4 \mathrm{~mL})$ and saturated aqueous $\mathrm{NaHCO}_{3}$ solution $(12 \mathrm{~mL})$ added. The reaction was warmed to $\mathrm{rt}$, and another further addition of benzyl chloroformate and $\mathrm{NaHCO}_{3}$ solution was made after $24 \mathrm{~h}$. After a further $24 \mathrm{~h}$, the solvent was removed in vacuo, and the aqueous layer extracted with EtOAc $(3 \times 50 \mathrm{~mL})$. The organic layer was washed with brine, dried over $\mathrm{Na}_{2} \mathrm{SO}_{4}$, filtered, and concentrated. The crude material was purified using chromatography eluting with 20\% EtOAc in hexanes to give an inseparable mixture of the product and benzyl alcohol. The mixture was heated to $55{ }^{\circ} \mathrm{C}$ at $0.12 \mathrm{~mm} \mathrm{Hg}$ until most of the benzyl alcohol had been removed. The product was obtained as a colourless oil (containing $20 \% \mathrm{BnOH}$ ), which was directly used in the next step without further purification. Corrected yield $(0.831 \mathrm{~g}, 80 \%) .{ }^{1} \mathrm{H}$ NMR (400 MHz, $\left.\mathrm{CDCl}_{3}\right) \delta 8.38(1 \mathrm{H}, \mathrm{dd}, J=4.6,1.3 \mathrm{~Hz}), 8.34(1 \mathrm{H}, \mathrm{br}$ s), 7.45-7.34 (6H, 
m), $6.89(1 \mathrm{H}, \mathrm{s}), 6.59\left(1 \mathrm{H}\right.$, br s), $5.23(2 \mathrm{H}, \mathrm{s})$. HRMS (ESI) calc'd for $\mathrm{C}_{15} \mathrm{H}_{13} \mathrm{~N}_{2} \mathrm{O}_{2} \mathrm{Cl}_{2}$ $\left([\mathrm{M}+\mathrm{H}]^{+}\right):$323.0348. Found: 323.0353 .

mCPBA (1.05 g, $4.70 \mathrm{mmol})$ was added to a solution of the $\mathrm{CBz}$ protected substrate (0.831 g, $2.6 \mathrm{mmol})$ in DCM (26 mL) and stirred at $\mathrm{rt}$ for $14 \mathrm{~h}$. The solvent was removed in vacuo, then the residue taken up in $\mathrm{MeOH}$ and pre-adsorbed onto silica gel. The crude material was purified using chromatography eluting with $5 \% \mathrm{MeOH}$ in EtOAc to give the $N$-oxide as a white solid (0.604 g, 69\%). mp 128-130 ${ }^{\circ} \mathrm{C}$. IR (neat?, $\mathrm{cm}^{-1}$ ): 2921, 1728, 1541, 1432, 1242. ${ }^{1} \mathrm{H}$ NMR (400 MHz, $\left.\mathrm{CDCl}_{3}\right) \delta 8.08(1 \mathrm{H}, \mathrm{d}, J=8.6 \mathrm{~Hz}), 8.02(1 \mathrm{H}, \mathrm{dd}$, $J=6.4,0.9 \mathrm{~Hz}), 7.42-7.34(5 \mathrm{H}, \mathrm{m}), 7.23(1 \mathrm{H}, \mathrm{dd}, J=8.6,6.4 \mathrm{~Hz}), 6.84(1 \mathrm{H}, \mathrm{s}), 6.80(1 \mathrm{H}$, s), $5.23(2 \mathrm{H}, \mathrm{s}) .{ }^{13} \mathrm{C} \mathrm{NMR}\left(100 \mathrm{MHz}, \mathrm{CDCl}_{3}\right) \delta 153.2,136.0,135.4,134.6,134.5,129.7$, 128.9, 128.7, 125.3, 118.9, 117.5, 68.2. HRMS (ESI) calc'd for $\mathrm{C}_{15} \mathrm{H}_{13} \mathrm{~N}_{2} \mathrm{O}_{3} \mathrm{Cl}_{2}\left([\mathrm{M}+\mathrm{H}]^{+}\right)$ 339.0297. Found: 339.0299.

\section{[3-(2,2-Dichlorovinyl)-1-oxy-pyridin-4-yl]-carbamic acid tert-butyl ester (29)}

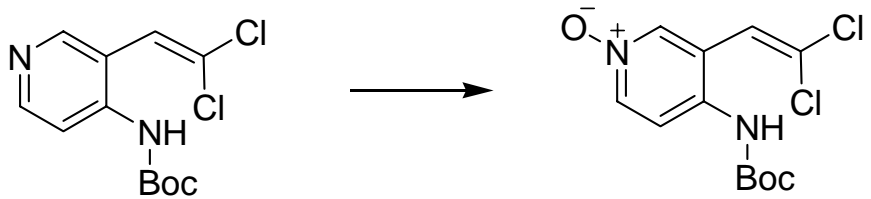

$m$ CPBA $(0.639 \mathrm{~g}, 2.86 \mathrm{mmol})$ was added to a solution of $10(0.450 \mathrm{~g}, 1.6 \mathrm{mmol})$ in DCM $(15 \mathrm{~mL})$, and stirred at $\mathrm{rt}$ for $18 \mathrm{~h}$. The solvent was removed in vacuo, then the residue taken up in $\mathrm{MeOH}$ and pre-adsorbed onto silica gel. The crude material was purified using chromatography eluting with $5 \% \rightarrow 10 \% \mathrm{MeOH}$ in EtOAc to give the $N$ oxide 29 as a white solid $(0.308 \mathrm{~g}, 63 \%)$. mp 136-138 ${ }^{\circ} \mathrm{C}$. IR (neat, $\mathrm{cm}^{-1}$ ): 2976, 1728, 1578, 1510, 1446, 1249, 1153. ${ }^{1} \mathrm{H}$ NMR (400 MHz, $\left.\mathrm{CDCl}_{3}\right) \delta 8.18(1 \mathrm{H}, \mathrm{s}), 8.10(2 \mathrm{H}, \mathrm{s})$, $6.61(1 \mathrm{H}, \mathrm{s}), 6.60(1 \mathrm{H}, \mathrm{s}), 1.54(9 \mathrm{H}, \mathrm{s}) .{ }^{13} \mathrm{C} \mathrm{NMR}\left(100 \mathrm{MHz}, \mathrm{CDCl}_{3}\right) \delta 151.7,139.4$, 139.0, 135.3, 129.9, 121.2, 119.2, 116.1, 83.0, 28.4. HRMS (ESI) calc'd for $\mathrm{C}_{12} \mathrm{H}_{15} \mathrm{~N}_{2} \mathrm{O}_{3} \mathrm{Cl}_{2}\left([\mathrm{M}+\mathrm{H}]^{+}\right)$305.0454. Found: 305.0466. 


\section{Preparation of Azaindoles}

\section{1-benzyl-2-phenyl-1H-pyrrolo[2,3-b]pyridine (6a)}

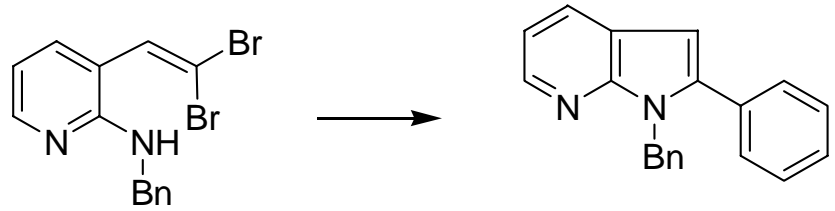

The general procedure for the tandem coupling was followed using 5 (88 $\mathrm{mg}, 0.24$ mmol), phenylboronic acid (44 mg, $0.36 \mathrm{mmol}), \mathrm{K}_{3} \mathrm{PO}_{4} \cdot \mathrm{H}_{2} \mathrm{O}$ (167 mg, $0.72 \mathrm{mmol}$ ), and a solution of $\mathrm{Pd}(\mathrm{OAc})_{2}(2.7 \mathrm{mg}, 0.012 \mathrm{mmol})$ and S-Phos (9.9 mg, $\left.0.024 \mathrm{mmol}\right)$ in PhMe $(2.5 \mathrm{~mL})$. The reaction mixture was heated at $100{ }^{\circ} \mathrm{C}$ for $2 \mathrm{~h}$. The crude material was purified using chromatography eluting with $25 \%$ EtOAc in hexanes to yield the product as a yellowish solid (50 mg, 73\%). mp 110-111 ${ }^{\circ} \mathrm{C} .{ }^{1} \mathrm{H}$ NMR (400 MHz, $\left.\mathrm{CDCl}_{3}\right) \delta 8.34$ $(1 \mathrm{H}, \mathrm{dd}, J=4.6,1.5 \mathrm{~Hz}), 7.92(1 \mathrm{H}, \mathrm{dd}, J=7.8,1.6 \mathrm{~Hz}), 7.40-7.35(5 \mathrm{H}, \mathrm{m}), 7.19-7.15$ $(3 \mathrm{H}, \mathrm{m}), 7.09(1 \mathrm{H}, \mathrm{dd}, J=7.7,4.7 \mathrm{~Hz}), 6.96-6.94(2 \mathrm{H}, \mathrm{m}), 6.55(1 \mathrm{H}, \mathrm{s}), 5.57(2 \mathrm{H}, \mathrm{s}) .{ }^{13} \mathrm{C}$ NMR (100 MHz, $\left.\mathrm{CDCl}_{3}\right) \delta 149.5,143.3,142.1,138.7,132.6,129.5,128.7,128.6,128.6$, 128.4, 127.2, 126.7, 120.8, 116.6, 100.5, 46.2. HRMS (EI) calc'd for $\mathrm{C}_{20} \mathrm{H}_{16} \mathrm{~N}_{2}\left([\mathrm{M}]^{+}\right)$ 284.1313. Found: 284.1315.

\section{1-benzyl-2-phenyl-1H-pyrrolo[2,3-b]pyridine (6a)}<smiles>ClC(Cl)=Cc1cccnc1NCc1ccccc1</smiles>

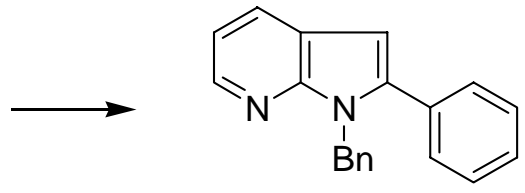

The general procedure for the tandem coupling was followed using 7 (83.5 $\mathrm{mg}, 0.30$ mmol), 2-phenylboronic acid (55 mg, $0.45 \mathrm{mmol}), \mathrm{K}_{3} \mathrm{PO}_{4} \cdot \mathrm{H}_{2} \mathrm{O}$ (350 mg, $0.15 \mathrm{mmol}$ ), and a solution of $\mathrm{Pd}(\mathrm{OAc})_{2}(2.3 \mathrm{mg}, 0.010 \mathrm{mmol})$ and $\mathrm{S}-\mathrm{Phos}(8.2 \mathrm{mg}, 0.020 \mathrm{mmol})$ in $\mathrm{PhMe}$ $(3 \mathrm{~mL})$. The reaction mixture was heated at $100{ }^{\circ} \mathrm{C}$ for $3 \mathrm{~h}$. The crude material was purified using chromatography eluting with $25 \%$ EtOAc/hexane to afford the product as a white solid (77 mg, 90\%).

\section{1-methyl-2-(4-trifluoromethylphenyl)-1H-pyrrolo[2,3-b]pyridine (6c)}



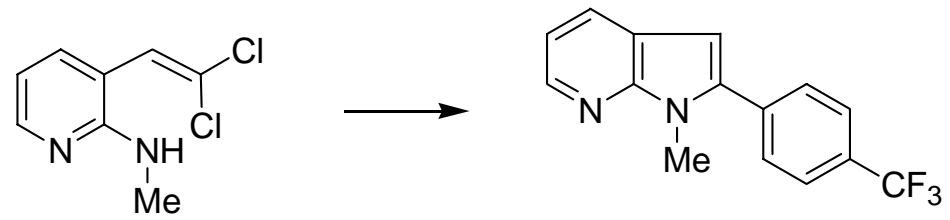

The general procedure for the tandem coupling was followed using 19 (49 mg, 0.24 mmol), 4-trifluoromethylphenylboronic acid (68 mg, $0.36 \mathrm{mmol}), \mathrm{K}_{3} \mathrm{PO}_{4} \cdot \mathrm{H}_{2} \mathrm{O}(167 \mathrm{mg}$, $0.72 \mathrm{mmol})$, and a solution of $\mathrm{Pd}(\mathrm{OAc})_{2}(2.7 \mathrm{mg}, 0.012 \mathrm{mmol})$ and S-Phos $(9.9 \mathrm{mg}, 0.024$ mmol) in PhMe $(2.5 \mathrm{~mL})$. The reaction mixture was heated at $100{ }^{\circ} \mathrm{C}$ for $2 \mathrm{~h}$. The crude material was purified using chromatography eluting with $25 \%$ EtOAc in hexanes to yield the product as an off- white solid (57 mg, 86\%). mp 105-106 ${ }^{\circ} \mathrm{C}$. IR (neat, $\mathrm{cm}^{-1}$ ): 2956, 1728, 1585, 1503, 1446. ${ }^{1} \mathrm{H}$ NMR (400 MHz, $\left.\mathrm{CDCl}_{3}\right) \delta 8.40(1 \mathrm{H}, \mathrm{dd}, J=4.8,1.5 \mathrm{~Hz})$, $7.94(1 \mathrm{H}, \mathrm{dd}, J=7.9,1.5 \mathrm{~Hz}), 7.77(2 \mathrm{H}, \mathrm{d}, J=8.3 \mathrm{~Hz}), 7.69(2 \mathrm{H}, \mathrm{d}, J=8.3 \mathrm{~Hz}), 7.12$ $(1 \mathrm{H}, \mathrm{dd}, J=7.9,4.8 \mathrm{~Hz}), 6.60(1 \mathrm{H}, \mathrm{s}), 3.91(3 \mathrm{H}, \mathrm{s}) \cdot{ }^{13} \mathrm{C} \mathrm{NMR}\left(100 \mathrm{MHz}, \mathrm{CDCl}_{3}\right) \delta$ $149.7,143.6,140.3,136.2,130.4\left(\mathrm{q}, J_{\mathrm{C}-\mathrm{F}}=33.0 \mathrm{~Hz}\right), 129.5,128.8,125.8\left(\mathrm{q}, J_{\mathrm{C}-\mathrm{F}}=3.8\right.$ $\mathrm{Hz}), 124.3\left(\mathrm{q}, J_{\mathrm{C}-\mathrm{F}}=272.7 \mathrm{~Hz}\right), 120.6,116.6,100.8,30.1 .{ }^{19} \mathrm{~F} \mathrm{NMR}\left(376 \mathrm{MHz}, \mathrm{CDCl}_{3}\right) \delta$ -62.6. HRMS (EI) calc'd for $\mathrm{C}_{15} \mathrm{H}_{11} \mathrm{~N}_{2} \mathrm{~F}_{3}\left([\mathrm{M}]^{+}\right)$276.0874. Found: 276.0870.

\section{1-methyl-2-(4-methoxyphenyl)-1H-pyrrolo[2,3-b]pyridine (6d)}<smiles>CNc1ncccc1C=C(Cl)Cl</smiles>

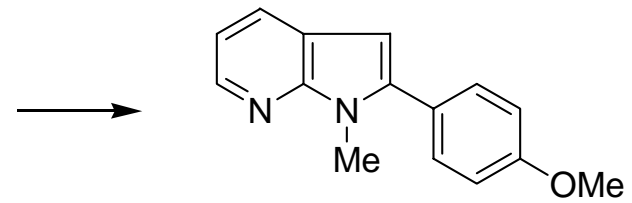

The general procedure for the tandem coupling was followed using 19 (49 mg, 0.24 mmol), 4-methoxyphenylboronic acid (55 mg, $0.36 \mathrm{mmol}), \mathrm{K}_{3} \mathrm{PO}_{4} \cdot \mathrm{H}_{2} \mathrm{O}(167 \mathrm{mg}, 0.72$ $\mathrm{mmol})$, and a solution of $\mathrm{Pd}(\mathrm{OAc})_{2}(2.7 \mathrm{mg}, 0.012 \mathrm{mmol})$ and S-Phos $(9.9 \mathrm{mg}, 0.024$ mmol) in PhMe $(2.5 \mathrm{~mL})$. The reaction mixture was heated at $100{ }^{\circ} \mathrm{C}$ for $1 \mathrm{~h}$. The crude material was purified using chromatography eluting with 25\% EtOAc in hexanes to yield the product as a white solid (48 $\mathrm{mg}, 84 \%$ ). $\mathrm{mp} 74-75^{\circ} \mathrm{C}$. IR (neat, $\mathrm{cm}^{-1}$ ): 2936, 1605, 1489, 1247. ${ }^{1} \mathrm{H}$ NMR (400 MHz, $\left.\mathrm{CDCl}_{3}\right) \delta 8.34(1 \mathrm{H}, \mathrm{dd}, J=4.6,1.3 \mathrm{~Hz}), 7.89(1 \mathrm{H}, \mathrm{dd}, J$ $=7.7,1.5 \mathrm{~Hz}), 7.49(2 \mathrm{H}, \mathrm{d}, J=8.8 \mathrm{~Hz}), 7.08(1 \mathrm{H}, \mathrm{dd}, J=7.9,4.8 \mathrm{~Hz}), 7.03(2 \mathrm{H}, \mathrm{d}, J=$ $8.8 \mathrm{~Hz}), 6.47(1 \mathrm{H}, \mathrm{s}), 3.89(3 \mathrm{H}, \mathrm{s}), 3.88(3 \mathrm{H}, \mathrm{s}) .{ }^{13} \mathrm{C} \mathrm{NMR}\left(100 \mathrm{MHz}, \mathrm{CDCl}_{3}\right) \delta 160.0$, 
149.3, 142.5, 142.0, 130.6, 128.1, 124.9, 121.0, 116.2, 114.3, 99.0, 55.6, 30.0. HRMS (EI) calc'd for $\mathrm{C}_{15} \mathrm{H}_{14} \mathrm{~N}_{2} \mathrm{O}\left([\mathrm{M}]^{+}\right)$: 238.1106. Found 238.1106.

\section{2-Phenylpyrrolo[2,3-b]pyridine-1-carboxylic acid tert-butyl ester (6e)}

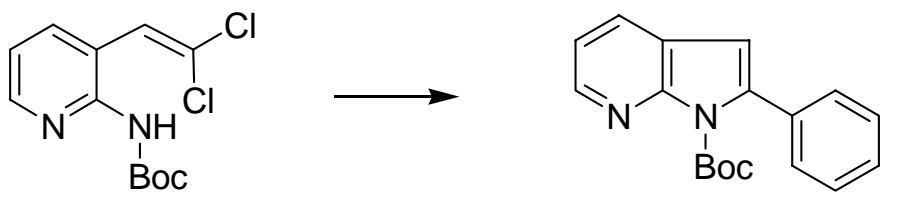

The general procedure for the tandem coupling was followed using 11 (70 mg, 0.24 mmol), phenylboronic acid (44 mg, $0.36 \mathrm{mmol}), \mathrm{K}_{3} \mathrm{PO}_{4} \cdot \mathrm{H}_{2} \mathrm{O}(167 \mathrm{mg}, 0.72 \mathrm{mmol})$, and a solution of $\mathrm{Pd}(\mathrm{OAc})_{2}(2.7 \mathrm{mg}, 0.012 \mathrm{mmol})$ and S-Phos $(9.9 \mathrm{mg}, 0.024 \mathrm{mmol})$ in PhMe $(2.5 \mathrm{~mL})$. The reaction mixture was heated at $100{ }^{\circ} \mathrm{C}$ for $4 \mathrm{~h}$. The crude material was purified using flash chromatography eluting with $25 \%$ EtOAc in hexanes to yield the product as a yellow solid (44 $\mathrm{mg}, 62 \%$ ). $\mathrm{mp} 121-122{ }^{\circ} \mathrm{C}$. IR (neat, $\mathrm{cm}^{-1}$ ): 2978, 1749, 1306, 1250, 1158. ${ }^{1} \mathrm{H}$ NMR $\left(400 \mathrm{MHz}, \mathrm{CDCl}_{3}\right) \delta 8.51(1 \mathrm{H}, \mathrm{dd}, J=4.8,1.8 \mathrm{~Hz}), 7.86$ $(1 \mathrm{H}, \mathrm{dd}, J=8.4,1.5 \mathrm{~Hz}), 7.46-7.36(5 \mathrm{H}, \mathrm{m}), 7.19(1 \mathrm{H}, \mathrm{dd}, J=7.7,4.6 \mathrm{~Hz}), 6.49(1 \mathrm{H}, \mathrm{s})$, $1.27(9 \mathrm{H}, \mathrm{s}) .{ }^{13} \mathrm{C}$ NMR $\left(100 \mathrm{MHz}, \mathrm{CDCl}_{3}\right) \delta 150.1,148.6,145.1,140.9,134.5,128.7$, 128.3, 122.0, 118.9, 106.1, 84.2, 27.6. HRMS (ESI) calc'd for $\mathrm{C}_{18} \mathrm{H}_{18} \mathrm{~N}_{2} \mathrm{O}_{2} \mathrm{Na}\left([\mathrm{M}+\mathrm{Na}]^{+}\right)$: 317.1260. Found: 317.1268 .

\section{3-methyl-2-phenylpyrrolo[2,3-b]pyridine-1-carboxylic acid tert-butyl ester (6f) and} 3-methyl-2-phenyl-1H-pyrrolo[2,3-b]pyridine (6ff)<smiles>CC(=C(Cl)Cl)c1cccnc1NC(=O)OCc1ccccc1</smiles>

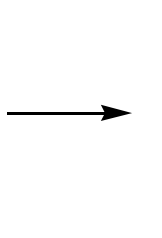<smiles>Cc1c(-c2ccccc2)n(C(=O)OC(C)(C)C)c2ncccc12</smiles>

$6 f$<smiles>Cc1c(-c2ccccc2)[nH]c2ncccc12</smiles>

$6 \mathrm{ff}$

The general procedure for the tandem coupling was followed using 20 (90.9 mg, 0.30 mmol), phenylboronic acid (55 mg, $0.45 \mathrm{mmol}), \mathrm{K}_{3} \mathrm{PO}_{4} \cdot \mathrm{H}_{2} \mathrm{O}(350 \mathrm{mg}, 1.5 \mathrm{mmol})$, and a solution of $\mathrm{Pd}(\mathrm{OAc})_{2}(2.3 \mathrm{mg}, 0.010 \mathrm{mmol})$ and $\mathrm{S}-\mathrm{Phos}(8.2 \mathrm{mg}, 0.020 \mathrm{mmol})$ in $\mathrm{PhMe}$ ( $3 \mathrm{~mL}$ ). The crude material was separated using flash chromatography, eluting with 25\% EtOAc in hexanes to yield $\mathbf{6 f}$ as a white solid (37 mg, 40\%) and $\mathbf{6 f f}$ as a white solid (36 mg, 57\%). 
6f: $\mathrm{mp} 148-150^{\circ} \mathrm{C}$. IR (neat, $\mathrm{cm}^{-1}$ ): 2980, 1747, $1415,1328,1250,1154 .{ }^{1} \mathrm{H}$ NMR $\left(400 \mathrm{MHz}, \mathrm{CDCl}_{3}\right) \delta 8.53(1 \mathrm{H}, \mathrm{dd}, J=4.9,1.4 \mathrm{~Hz}), 7.83(1 \mathrm{H}, \mathrm{dd}, J=7.8,1.5 \mathrm{~Hz}), 7.48-$ $7.34(5 \mathrm{H}, \mathrm{m}), 7.21(1 \mathrm{H}, \mathrm{dd}, J=7.6,4.8 \mathrm{~Hz}), 2.15(3 \mathrm{H}, \mathrm{s}), 1.20(9 \mathrm{H}, \mathrm{s}) .{ }^{13} \mathrm{C}$ NMR $(75$ $\left.\mathrm{MHz}, \mathrm{CDCl}_{3}\right) \delta 149.5,148.7,145.2,136.1,133.9,129.8,128.2,127.9,127.1,123.2$, 118.5, 113.2, 83.6, 27.5, 9.2. HRMS (ESI) calc'd for $\mathrm{C}_{19} \mathrm{H}_{20} \mathrm{~N}_{2} \mathrm{O}_{2} \mathrm{Na}\left([\mathrm{M}+\mathrm{Na}]^{+}\right)$: 331.1416. Found: 331.1428 .

6ff: $\mathrm{mp} 199-200{ }^{\circ} \mathrm{C}$. IR (neat, $\mathrm{cm}^{-1}$ ): 3433, 2914, 1580, 1443, 1289. ${ }^{1} \mathrm{H}$ NMR (400 $\left.\mathrm{MHz}, \mathrm{CDCl}_{3}\right) \delta 11.12(1 \mathrm{H}, \mathrm{br}), 8.22(1 \mathrm{H}, \mathrm{dd}, J=4.9,1.5 \mathrm{~Hz}), 7.91(1 \mathrm{H}, \mathrm{dd}, J=7.8,1.4$ $\mathrm{Hz}), 7.73(2 \mathrm{H}, \mathrm{d}, J=7.2 \mathrm{~Hz}), 7.54(2 \mathrm{H}, \mathrm{t}, J=7.6 \mathrm{~Hz}), 7.41(1 \mathrm{H}, \mathrm{t}, J=7.4 \mathrm{~Hz}), 7.08(1 \mathrm{H}$, $\mathrm{dd}, J=7.8,4.8 \mathrm{~Hz}), 2.49(3 \mathrm{H}, \mathrm{s}) .{ }^{13} \mathrm{C}$ NMR $\left(100 \mathrm{MHz}, \mathrm{CDCl}_{3}\right) \delta 149.2,142.4,135.4$, 133.4, 129.0, 128.4, 127.8, 127.3, 123.2, 115.4, 106.5, 9.9. HRMS (ESI) calc'd for $\mathrm{C}_{14} \mathrm{H}_{13} \mathrm{~N}_{2}\left([\mathrm{M}+\mathrm{H}]^{+}\right)$: 209.1073. Found: 209.1072 .

\section{2-o-tolylpyrrolo[2,3-c]pyridine-1-carboxylic acid tert-butyl ester (24b)}

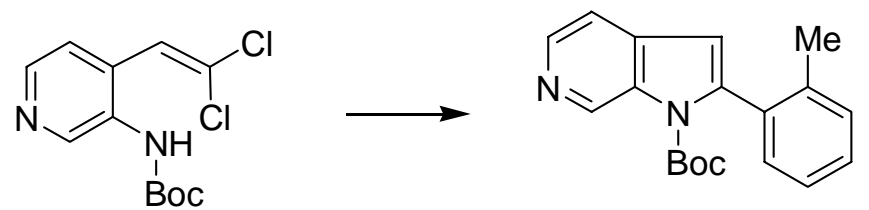

The general procedure for the tandem coupling was followed using 14 (70 mg, 0.24 mmol), o-tolylboronic acid (44 mg, $0.36 \mathrm{mmol}), \mathrm{K}_{3} \mathrm{PO}_{4} \cdot \mathrm{H}_{2} \mathrm{O}(167 \mathrm{mg}, 0.73 \mathrm{mmol}$ ), and a solution of $\mathrm{Pd}(\mathrm{OAc})_{2}(2.7 \mathrm{mg}, 0.012 \mathrm{mmol})$ and S-Phos $(9.9 \mathrm{mg}, 0.024 \mathrm{mmol})$ in PhMe $(2.5 \mathrm{~mL})$. The reaction mixture was heated at $100{ }^{\circ} \mathrm{C}$ for $8 \mathrm{~h}$. The crude material was purified using chromatography eluting with $25 \%$ EtOAc in hexanes to afford the product as a colorless oil (54 mg, 73\%). IR (neat, $\mathrm{cm}^{-1}$ ): 2928, 1732, 1589, 1432, 1340, 1147. ${ }^{1} \mathrm{H}$ NMR (400 MHz, $\left.\mathrm{CDCl}_{3}\right) \delta 9.53(1 \mathrm{H}, \mathrm{s}), 8.45(1 \mathrm{H}, \mathrm{d}, J=5.3 \mathrm{~Hz}), 7.49(1 \mathrm{H}, \mathrm{dd}, J=5.3$, $0.9 \mathrm{~Hz}), 7.37-7.32(1 \mathrm{H}, \mathrm{m}), 7.29-7.23(3 \mathrm{H}, \mathrm{m}), 6.47(1 \mathrm{H}, \mathrm{d}, J=0.6 \mathrm{~Hz}), 2.18(3 \mathrm{H}, \mathrm{s})$, $1.30(9 \mathrm{H}, \mathrm{s}) .{ }^{13} \mathrm{C}$ NMR $\left(100 \mathrm{MHz}, \mathrm{CDCl}_{3}\right) \delta 149.4,143.0,142.4,138.0,137.2,134.8$, 134.2, 133.9, 129.8, 129.7, 128.9, 125.6, 114.5, 108.6, 84.3, 27.7, 20.1. HRMS (ESI) calc'd for $\mathrm{C}_{19} \mathrm{H}_{21} \mathrm{~N}_{2} \mathrm{O}_{2}\left([\mathrm{M}+\mathrm{H}]^{+}\right)$): 309.1597. Found: 309.1605. 


\section{2-(4-trifluoromethylphenyl)pyrrolo[2,3-c]pyridine-1-carboxylic acid tert-butyl ester} (24c)
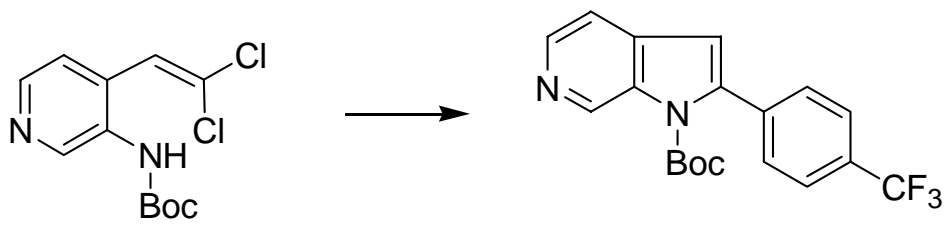

The general procedure for the tandem coupling was followed using 14 (70 mg, 0.24 mmol), 4-trifluoromethylphenylboronic acid (69 mg, $0.36 \mathrm{mmol}), \mathrm{K}_{3} \mathrm{PO}_{4} \cdot \mathrm{H}_{2} \mathrm{O}(167 \mathrm{mg}$, $0.73 \mathrm{mmol})$, and a solution of $\mathrm{Pd}(\mathrm{OAc})_{2}(2.7 \mathrm{mg}, 0.012 \mathrm{mmol})$ and S-Phos $(9.9 \mathrm{mg}, 0.024$ mmol) in PhMe $(2.5 \mathrm{~mL})$. The reaction mixture was heated at $100{ }^{\circ} \mathrm{C}$ for $8 \mathrm{~h}$. The crude material was purified using chromatography eluting with $50 \% \rightarrow 70 \%$ EtOAc/hexane to afford the product as a colorless oil (63 mg, 72\%). IR (neat, $\mathrm{cm}^{-1}$ ): 2990, 1735, 1592, 1324, 1133. ${ }^{1} \mathrm{H}$ NMR $\left(400 \mathrm{MHz}, \mathrm{CDCl}_{3}\right) \delta 9.48(1 \mathrm{H}, \mathrm{s}), 8.45(1 \mathrm{H}, \mathrm{d}, J=5.3 \mathrm{~Hz}), 7.70$ $(2 \mathrm{H}, \mathrm{d}, J=8.1 \mathrm{~Hz}), 7.57(2 \mathrm{H}, \mathrm{d}, J=7.9 \mathrm{~Hz}), 7.49(1 \mathrm{H}, \mathrm{dd}, J=5.3,1.1 \mathrm{~Hz}), 6.61(1 \mathrm{H}, \mathrm{s})$, $1.40(9 \mathrm{H}, \mathrm{s}) .{ }^{13} \mathrm{C}$ NMR $\left(100 \mathrm{MHz}, \mathrm{CDCl}_{3}\right) \delta 149.3,142.7,142.3,138.2,137.6,134.5$, $134.4,130.7\left(\mathrm{q}, J_{\mathrm{C}-\mathrm{F}}=33.0 \mathrm{~Hz}\right), 129.5,125.1\left(\mathrm{q}, J_{\mathrm{C}-\mathrm{F}}=3.8 \mathrm{~Hz}\right), 124.2\left(\mathrm{q}, J_{\mathrm{C}-\mathrm{F}}=272.2\right.$ $\mathrm{Hz}), 115.2,109.9,85.2,27.9 .{ }^{19} \mathrm{~F}$ NMR $\left(376 \mathrm{MHz}, \mathrm{CDCl}_{3}\right) \delta-62.2$. HRMS (ESI) calc'd for $\mathrm{C}_{19} \mathrm{H}_{18} \mathrm{~N}_{2} \mathrm{O}_{2} \mathrm{~F}_{3}\left([\mathrm{M}+\mathrm{H}]^{+}\right)$: 363.1314 . Found: 363.1329 .

\section{2-(4-methoxyphenyl)pyrrolo[2,3-c]pyridine-1-carboxylic acid tert-butyl ester (24d)}<smiles>CC(C)(C)OC(=O)Nc1cnccc1C=C(Cl)Cl</smiles><smiles>COc1ccc(-c2cc3ccncc3n2C(=O)OC(C)(C)C)cc1</smiles>

The general procedure for the tandem coupling was followed using 14 (70 mg, 0.24 mmol), 4-methoxyphenylboronic acid (55 mg, $0.36 \mathrm{mmol}), \mathrm{K}_{3} \mathrm{PO}_{4} \cdot \mathrm{H}_{2} \mathrm{O}(167 \mathrm{mg}, 0.73$ $\mathrm{mmol})$, and a solution of $\mathrm{Pd}(\mathrm{OAc})_{2}(2.7 \mathrm{mg}, 0.012 \mathrm{mmol})$ and S-Phos $(9.9 \mathrm{mg}, 0.024$ mmol) in PhMe $(2.5 \mathrm{~mL})$. The reaction mixture was heated at $100^{\circ} \mathrm{C}$ for $1.5 \mathrm{~h}$. The crude material was purified using chromatography eluting with $30 \% \rightarrow 35 \% \rightarrow 40 \%$ EtOAc in hexanes to afford the product as a white solid (63 $\mathrm{mg}, 81 \%$ ). $\mathrm{mp} 103-104{ }^{\circ} \mathrm{C}$. IR (neat, 
$\left.\mathrm{cm}^{-1}\right): 2983,1735,1599,1494,1432,1330,1252,1140 .{ }^{1} \mathrm{H} \mathrm{NMR}\left(400 \mathrm{MHz}, \mathrm{CDCl}_{3}\right) \delta$ $9.44(1 \mathrm{H}, \mathrm{s}), 8.42(1 \mathrm{H}, \mathrm{d}, J=5.3 \mathrm{~Hz}), 7.46(1 \mathrm{H}, \mathrm{d}, J=5.3 \mathrm{~Hz}), 7.37(2 \mathrm{H}, \mathrm{d}, J=8.8 \mathrm{~Hz})$, $6.97(2 \mathrm{H}, \mathrm{d}, J=8.6 \mathrm{~Hz}), 6.52(1 \mathrm{H}, \mathrm{s}), 3.87(3 \mathrm{H}, \mathrm{s}), 1.44(9 \mathrm{H}, \mathrm{s}) .{ }^{13} \mathrm{C}$ NMR $(100 \mathrm{MHz}$, $\left.\mathrm{CDCl}_{3}\right) \delta 160.1,149.6,144.1,142.4,137.8,134.8,134.3,130.3,126.2,114.8,113.6$, 108.5, 84.8, 55.6, 28.0. HRMS (ESI) calc'd for $\mathrm{C}_{19} \mathrm{H}_{21} \mathrm{~N}_{2} \mathrm{O}_{3}\left([\mathrm{M}+\mathrm{H}]^{+}\right)$: 325.1546. Found: 325.1562 .

\section{2-naphthalen-2-ylpyrrolo[2,3-c]pyridine-1-carboxylic acid tert-butyl ester (24e)}

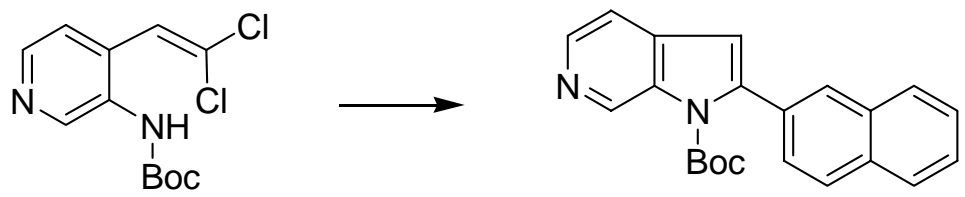

The general procedure for the tandem coupling was followed using 14 (87 mg, 0.30 mmol), 2-naphthylboronic acid (77 mg, $0.45 \mathrm{mmol}), \mathrm{K}_{3} \mathrm{PO}_{4} \cdot \mathrm{H}_{2} \mathrm{O}(207 \mathrm{mg}, 0.90 \mathrm{mmol})$, and a solution of $\mathrm{Pd}(\mathrm{OAc})_{2}(2.0 \mathrm{mg}, 0.009 \mathrm{mmol})$ and S-Phos $(7.4 \mathrm{mg}, 0.018 \mathrm{mmol})$ in $\mathrm{PhMe}(1.5 \mathrm{~mL})$. The reaction mixture was heated at $100{ }^{\circ} \mathrm{C}$ for $5 \mathrm{~h}$. The crude material was purified using chromatography eluting with 50\% EtOAc in hexanes to afford the product as a yellow oil (72.8 mg, 70\%). IR (neat, $\mathrm{cm}^{-1}$ ): 2921, 1731, 1585, 1432, 1327, 1143. ${ }^{1} \mathrm{H}$ NMR (400 MHz, $\left.\mathrm{CDCl}_{3}\right) \delta 9.51(1 \mathrm{H}, \mathrm{s}), 8.46(1 \mathrm{H}, \mathrm{d}, J=5.3 \mathrm{~Hz}), 7.96(1 \mathrm{H}, \mathrm{s})$, 7.92-7.86 (3H, m), 7.57- $7.49(4 \mathrm{H}, \mathrm{m}), 6.67(1 \mathrm{H}, \mathrm{s}), 1.34(9 \mathrm{H}, \mathrm{s}) .{ }^{13} \mathrm{C} \mathrm{NMR}(100 \mathrm{MHz}$, $\left.\mathrm{CDCl}_{3}\right) \delta 149.4,143.9,142.3,137.7,134.6,133.0,132.9,131.1,128.1,127.8,127.6$, 127.3, 126.9, 126.6, 126.6, 114.8, 109.1, 84.8, 27.6. HRMS (ESI) calc'd for $\mathrm{C}_{22} \mathrm{H}_{21} \mathrm{~N}_{2} \mathrm{O}_{2}$ $\left([\mathrm{M}+\mathrm{H}]^{+}\right): 345.1597$. Found: 345.1595 .

\section{2-thiophen-3-yl-pyrrolo[2,3-c]pyridine-1-carboxylic acid tert-butyl ester (24f)}

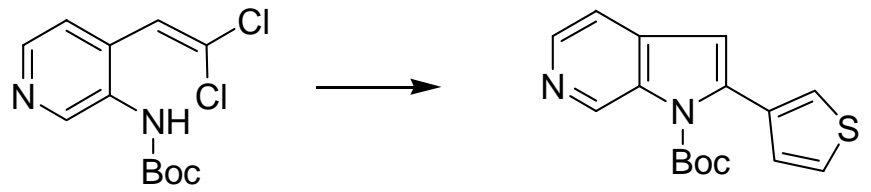

The general procedure for the tandem coupling was followed using 14 (70 mg, 0.24 mmol), 3-thienoboronic acid (46 mg, $0.36 \mathrm{mmol}), \mathrm{K}_{3} \mathrm{PO}_{4} \cdot \mathrm{H}_{2} \mathrm{O}(167 \mathrm{mg}, 0.73 \mathrm{mmol})$, and 
a solution of $\mathrm{Pd}(\mathrm{OAc})_{2}(2.7 \mathrm{mg}, 0.012 \mathrm{mmol})$ and $\mathrm{S}-\mathrm{Phos}(9.9 \mathrm{mg}, 0.024 \mathrm{mmol})$ in $\mathrm{PhMe}$ $(2.5 \mathrm{~mL})$. The reaction mixture was heated at $100{ }^{\circ} \mathrm{C}$ for $20 \mathrm{~h}$. The crude material was purified using chromatography eluting with $10 \% \rightarrow 20 \% \rightarrow 25 \%$ EtOAc in hexanes to afford the product as an off-white solid (40 mg, 55\%). $\mathrm{mp} 86-87^{\circ} \mathrm{C}$. IR (neat, $\mathrm{cm}^{-1}$ ): 2918, 1737, 1337, 1149. ${ }^{1} \mathrm{H}$ NMR (400 MHz, $\left.\mathrm{CDCl}_{3}\right) \delta 9.44(1 \mathrm{H}, \mathrm{s}), 8.41(1 \mathrm{H}, \mathrm{d}, J=5.3$ $\mathrm{Hz}), 7.45(1 \mathrm{H}, \mathrm{dd}, J=5.3,0.9 \mathrm{~Hz}), 7.41(1 \mathrm{H}, \mathrm{dd}, J=3.1,1.3 \mathrm{~Hz}), 7.36(1 \mathrm{H}, \mathrm{dd}, J=5.1$, $3.1 \mathrm{~Hz}), 7.17(1 \mathrm{H}, \mathrm{dd}, J=5.1,1.3 \mathrm{~Hz}), 6.58(1 \mathrm{H}, \mathrm{s}), 1.48(9 \mathrm{H}, \mathrm{s}) .{ }^{13} \mathrm{C}$ NMR $(100 \mathrm{MHz}$, $\left.\mathrm{CDCl}_{3}\right) \delta 149.5,142.4,139.0,137.9,134.6,134.2,133.8,129.0,125.2,124.5,114.9$, 109.2, 84.9, 28.0. HRMS (ESI) calc'd for $\mathrm{C}_{16} \mathrm{H}_{17} \mathrm{~N}_{2} \mathrm{O}_{2} \mathrm{~S}\left([\mathrm{M}+\mathrm{H}]^{+}\right)$: 301.1005. Found: 301.1016 .

\section{2-pent-1-enyl-pyrrolo[2,3-c]pyridine-1-carboxylic acid tert-butyl ester (24g)}

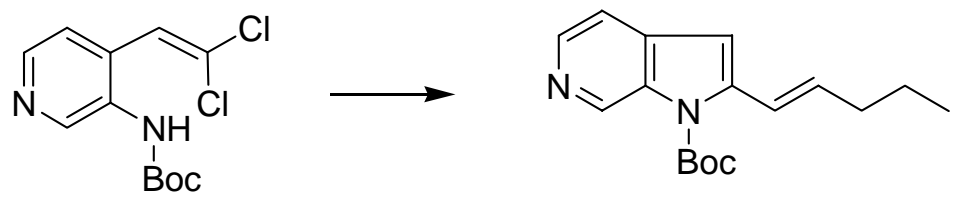

The general procedure for the tandem coupling was followed using 14 (70 mg, 0.24 mmol), 1-pentenylboronic acid (30 mg, $0.26 \mathrm{mmol}), \mathrm{K}_{3} \mathrm{PO}_{4} \cdot \mathrm{H}_{2} \mathrm{O}(167 \mathrm{mg}, 0.73 \mathrm{mmol}$ ), and a solution of $\mathrm{Pd}(\mathrm{OAc})_{2}(2.7 \mathrm{mg}, 0.012 \mathrm{mmol})$ and S-Phos $(9.9 \mathrm{mg}, 0.024 \mathrm{mmol})$ in PhMe $(3.4 \mathrm{~mL})$. The reaction mixture was heated at $100{ }^{\circ} \mathrm{C}$ for $2 \mathrm{~h}$. The crude material was purified using chromatography eluting with $25 \% \rightarrow 35 \%$ EtOAc in hexanes to afford the product as a colourless oil (53 mg, 79\%). IR (neat, $\mathrm{cm}^{-1}$ ): 2937, 1738, 1325, 1148. ${ }^{1} \mathrm{H}$ NMR (400 MHz, CD 3 OD) $\delta 9.20(1 \mathrm{H}, \mathrm{s}), 8.20(1 \mathrm{H}, \mathrm{d}, J=5.3 \mathrm{~Hz}), 7.49(1 \mathrm{H}, \mathrm{d}, J=5.3$ $\mathrm{Hz}), 6.96(1 \mathrm{H}, \mathrm{d}, J=15.8 \mathrm{~Hz}), 6.72(1 \mathrm{H}, \mathrm{s}), 6.36\left(1 \mathrm{H}, \mathrm{dt}, J^{\mathrm{d}}=15.6 \mathrm{~Hz}, J^{\mathrm{t}}=7.0 \mathrm{~Hz}\right), 2.54$ $(2 \mathrm{H}, \mathrm{q}, J=7.3 \mathrm{~Hz}), 1.70(9 \mathrm{H}, \mathrm{s}), 1.54(2 \mathrm{H}$, sextet, $J=7.3 \mathrm{~Hz}), 0.99(3 \mathrm{H}, \mathrm{t}, J=7.5 \mathrm{~Hz})$. ${ }^{13} \mathrm{C}$ NMR (100 MHz, CD 3 OD) $\delta 151.0,145.6,142.1,138.4,137.8,137.1,135.0,122.7$, 116.3, 105.6, 86.8, 36.5, 28.5, 23.4, 14.2. HRMS (EI) calc'd for $\mathrm{C}_{17} \mathrm{H}_{22} \mathrm{~N}_{2} \mathrm{O}_{2} \mathrm{~S}\left([\mathrm{M}]^{+}\right)$: 286.1681. Found: 286.1683.

2-(2-methoxyquinolin-3-yl)-pyrrolo[2,3-c]pyridine-1-carboxylic acid tert-butyl ester (24h) 

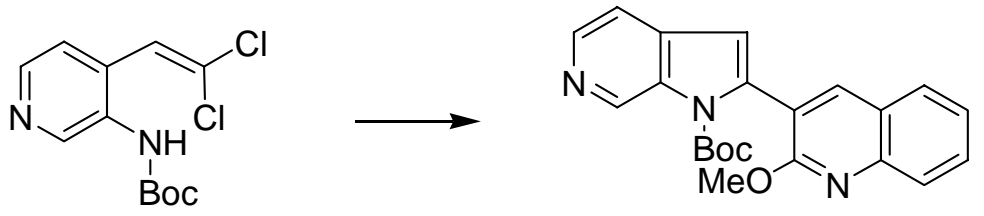

The general procedure for the tandem coupling was followed using $14(87 \mathrm{mg}, 0.30$ mmol), 2-methoxy-3-quinolin-3-ylboronic acid (91 mg, $0.45 \mathrm{mmol}$ ), $\mathrm{K}_{3} \mathrm{PO}_{4} \cdot \mathrm{H}_{2} \mathrm{O}$ (207 $\mathrm{mg}, 0.90 \mathrm{mmol})$, and a solution of $\mathrm{Pd}(\mathrm{OAc})_{2}(3.4 \mathrm{mg}, 0.015 \mathrm{mmol})$ and S-Phos $(12.3 \mathrm{mg}$, $0.030 \mathrm{mmol})$ in $\mathrm{PhMe}(3 \mathrm{~mL})$. The reaction mixture was heated at $100{ }^{\circ} \mathrm{C}$ for $1 \mathrm{~h}$. The crude material was purified using chromatography eluting with $40 \rightarrow 50 \%$ EtOAc in hexane to give the product as a white solid $(102 \mathrm{mg}, 91 \%)$. $\mathrm{mp} 88-90^{\circ} \mathrm{C}$. IR (neat, $\mathrm{cm}^{-1}$ ): 2976, 1742, 1623, 1582, 1456, 1344, 1266, 1147. ${ }^{1} \mathrm{H}$ NMR (400 MHz, $\left.\mathrm{CDCl}_{3}\right) \delta 9.50$ $(1 \mathrm{H}, \mathrm{s}), 8.44(1 \mathrm{H}, \mathrm{d}, J=5.3 \mathrm{~Hz}), 8.05(1 \mathrm{H}, \mathrm{s}), 7.89(1 \mathrm{H}, \mathrm{d}, J=8.1 \mathrm{~Hz}), 7.77(1 \mathrm{H}, \mathrm{dd}, J=$ $8.13 \mathrm{~Hz}), 7.66(1 \mathrm{H}$, ddd, $J=8.6,7.0,1.5 \mathrm{~Hz}), 7.50(1 \mathrm{H}, \mathrm{dd}, J=5.3,1.1 \mathrm{~Hz}), 7.43(1 \mathrm{H}$, ddd, $J=8.1,7.0,1.1 \mathrm{~Hz}), 6.37(1 \mathrm{H}, \mathrm{s}), 4.05(3 \mathrm{H}, \mathrm{s}), 1.37(9 \mathrm{H}, \mathrm{s}) .{ }^{13} \mathrm{C} \mathrm{NMR}(100 \mathrm{MHz}$, $\left.\mathrm{CDCl}_{3}\right) \delta 159.8,149.4,146.6,142.2,139.2,137.9,137.5,134.5,134.1,130.2,127.8$, $127.4,124.9,124.7,120.0,115.1,109.4,84.8,53.9,27.9$. HRMS (ESI) calc'd for $\mathrm{C}_{22} \mathrm{H}_{22} \mathrm{~N}_{3} \mathrm{O}_{3}\left([\mathrm{M}+\mathrm{H}]^{+}\right)$: 376.1655 . Found: 376.1663 .

\section{2-Phenylpyrrolo[3,2-c]pyridine-1-carboxylic acid tert-butyl ester (27a)}



The general procedure for the tandem coupling was followed using 10 (58 $\mathrm{mg}, 0.2$ mmol), phenylboronic acid (29.3 mg, $0.24 \mathrm{mmol}), \mathrm{K}_{3} \mathrm{PO}_{4} \cdot \mathrm{H}_{2} \mathrm{O}(230 \mathrm{mg}, 1 \mathrm{mmol}$ ), $\mathrm{Pd}(\mathrm{OAc})_{2}(2.3 \mathrm{mg}, 0.01 \mathrm{mmol})$, and Buchwald family of bipehnylphosphine ligand (0.02 mmol) indicated in Table 3-4. The reaction mixture was heated in PhMe (2 mL) at 100 ${ }^{\circ} \mathrm{C}$ for $4.5 \mathrm{~h}$. The crude material after workup was purified using chromatography eluting with $33 \%$ EtOAc in hexanes to give the desired product as a white solid $(48.3 \mathrm{mg}, 80 \%)$.

The use of S-Phos ( $8.2 \mathrm{mg}, 0.02 \mathrm{mmol})$ afforded $46.4 \mathrm{mg}$ of $27 \mathrm{a}$ (79\%).

The use of DavePhos (7.9 mg, $0.02 \mathrm{mmol}$ ) afforded $46.5 \mathrm{mg}$ of $27 \mathbf{a}(80 \%)$. 
The use of X-Phos (9.5 mg, $0.02 \mathrm{mmol}$ ) afforded $51.5 \mathrm{mg}$ of $27 \mathbf{a}(87 \%)$.

The use of dicyclohexyl-(2'-methylbiphenyl-2-yl)-phosphine $(7.3 \mathrm{mg}, 0.02 \mathrm{mmol})$ afforded $48.2 \mathrm{mg}$ of $27 \mathrm{a}(82 \%)$.

27a: $\mathrm{mp} 128-129{ }^{\circ} \mathrm{C}$. IR (neat, $\mathrm{cm}^{-1}$ ): $2979,1737,1459,1339,1321,1228,1152 .{ }^{1} \mathrm{H}$ NMR $\left(400 \mathrm{MHz}, \mathrm{CDCl}_{3}\right) \delta 8.88(1 \mathrm{H}, \mathrm{s}), 8.49(1 \mathrm{H}, \mathrm{d}, J=5.7 \mathrm{~Hz}), 8.05(1 \mathrm{H}, \mathrm{d}, J=5.7$ $\mathrm{Hz}), 7.43-7.40(5 \mathrm{H}, \mathrm{m}), 6.62(1 \mathrm{H}, \mathrm{s}), 1.32(9 \mathrm{H}, \mathrm{s}) .{ }^{13} \mathrm{C} \mathrm{NMR}\left(100 \mathrm{MHz}, \mathrm{CDCl}_{3}\right) \delta$ 149.7, 144.3, 143.5, 141.9, 141.6, 134.2, 129.0, 128.3, 128.1, 125.8, 110.3, 108.0, 84.7, 27.7. HRMS (ESI): calc'd for $\mathrm{C}_{18} \mathrm{H}_{19} \mathrm{~N}_{2} \mathrm{O}_{2}\left([\mathrm{M}+\mathrm{H}]^{+}\right)$295.1441. Found: 295.1453 .

\section{2-(4-fluorophenyl)pyrrolo[3,2-c]pyridine-1-carboxylic acid tert-butyl ester (27b)}

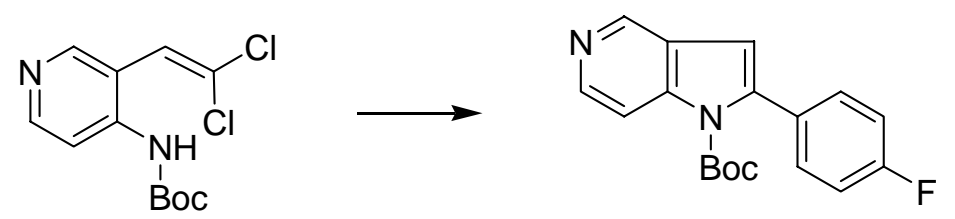

The general procedure for the tandem coupling was followed using 10 (58 $\mathrm{mg}, 0.2$ mmol), 4-fluorophenylboronic acid (33.6 mg, $0.24 \mathrm{mmol}$ ), $\mathrm{K}_{3} \mathrm{PO}_{4} \cdot \mathrm{H}_{2} \mathrm{O}(230 \mathrm{mg}, 1$ $\mathrm{mmol}), \mathrm{Pd}(\mathrm{OAc})_{2}(2.3 \mathrm{mg}, 0.01 \mathrm{mmol})$, and X-Phos $(9.5 \mathrm{mg}, 0.02 \mathrm{mmol})$. The reaction mixture was heated in $\mathrm{PhMe}(2 \mathrm{~mL})$ at $100{ }^{\circ} \mathrm{C}$ for $2.5 \mathrm{~h}$. The crude material after workup was purified using chromatography eluting with $30 \% \rightarrow 35 \%$ EtOAc in hexanes to give the desired product as a white solid ( $55 \mathrm{mg}, 88 \%$ ). $\mathrm{mp} 132-133{ }^{\circ} \mathrm{C}$. IR (neat, $\left.\mathrm{cm}^{-1}\right): 1728$, 1592, 1497, 1453, 1340, 1225, $1154 .{ }^{1} \mathrm{H}$ NMR $\left(400 \mathrm{MHz}, \mathrm{CDCl}_{3}\right) \delta 8.87(1 \mathrm{H}, \mathrm{d}, J=1.1$ $\mathrm{Hz}), 8.49(1 \mathrm{H}, \mathrm{d}, J=5.7 \mathrm{~Hz}), 8.03\left(1 \mathrm{H}, \mathrm{dt}, J^{\mathrm{d}}=5.7 \mathrm{~Hz}, J^{\mathrm{t}}=0.9 \mathrm{~Hz}\right), 7.42-7.36(2 \mathrm{H}, \mathrm{m})$, $7.12(2 \mathrm{H}, \mathrm{tm}, J=8.6 \mathrm{~Hz}), 6.59(1 \mathrm{H}, \mathrm{d}, J=0.9 \mathrm{~Hz}), 1.37(9 \mathrm{H}, \mathrm{s}) .{ }^{13} \mathrm{C}$ NMR $(100 \mathrm{MHz}$, $\left.\mathrm{CDCl}_{3}\right) \delta 162.9\left(J_{\mathrm{C}-\mathrm{F}}=248 \mathrm{~Hz}\right), 149.6,144.4,143.6,141.9,140.5,130.8\left(J_{\mathrm{C}-\mathrm{F}}=8.4 \mathrm{~Hz}\right)$, $130.3,125.7,115.2\left(J_{\mathrm{C}-\mathrm{F}}=21 \mathrm{~Hz}\right), 110.4,108.3,85.0,27.8 .{ }^{19} \mathrm{~F}$ NMR $(377 \mathrm{MHz}) \delta-$ 113.3. HRMS (EI): calc'd for $\mathrm{C}_{18} \mathrm{H}_{17} \mathrm{~N}_{2} \mathrm{O}_{2} \mathrm{~F}\left([\mathrm{M}]^{+}\right)$312.1274. Found: 312.1280 .

\section{2-(4-methoxyphenyl)pyrrolo[3,2-c]pyridine-1-carboxylic acid tert-butyl ester (27c)}






The general procedure for the tandem coupling was followed using 10 (58 $\mathrm{mg}, 0.2$ mmol), 4-methoxyphenylboronic acid (36 mg, $0.24 \mathrm{mmol}), \mathrm{K}_{3} \mathrm{PO}_{4} \cdot \mathrm{H}_{2} \mathrm{O}(230 \mathrm{mg}, 1$ $\mathrm{mmol}), \mathrm{Pd}(\mathrm{OAc})_{2}(2.3 \mathrm{mg}, 0.01 \mathrm{mmol})$, and S-Phos $(8.2 \mathrm{mg}, 0.02 \mathrm{mmol})$. The reaction mixture was heated in $\mathrm{PhMe}(2 \mathrm{~mL})$ at $100{ }^{\circ} \mathrm{C}$ for $15 \mathrm{~h}$. The crude material after workup was purified using chromatography eluting with $30 \% \rightarrow 40 \%$ EtOAc in hexanes to give the desired product as a colorless oil (44 mg, 68\%). IR (neat, $\mathrm{cm}^{-1}$ ): 2974, 1738, 1450, 1332, 1240, 1155. ${ }^{1} \mathrm{H}$ NMR (400 MHz, $\left.\mathrm{CDCl}_{3}\right) \delta 8.86(1 \mathrm{H}, \mathrm{d}, J=0.6 \mathrm{~Hz}), 8.47(1 \mathrm{H}, \mathrm{d}, J$ $=5.9 \mathrm{~Hz}), 8.02(1 \mathrm{H}, \mathrm{d}, J=5.9 \mathrm{~Hz}), 7.34\left(2 \mathrm{H}, \mathrm{dt}, J^{\mathrm{d}}=9.0 \mathrm{~Hz}, J^{\mathrm{d}}=2.2 \mathrm{~Hz}\right), 6.96(2 \mathrm{H}, \mathrm{dt}$, $\left.J^{\mathrm{d}}=8.8 \mathrm{~Hz}, J^{\mathrm{t}}=2.2 \mathrm{~Hz}\right), 6.56(1 \mathrm{H}, \mathrm{d}, J=0.7 \mathrm{~Hz}), 3.86(3 \mathrm{H}, \mathrm{s}), 1.38(9 \mathrm{H}, \mathrm{s}) .{ }^{13} \mathrm{C} \mathrm{NMR}$ $\left(100 \mathrm{MHz} \mathrm{CDCl}_{3}\right) \delta 159.9,149.8,144.1,143.4,141.8,141.6,130.3,126.5,125.9,113.6$, 110.3, 107.6, 84.7, 55.6, 27.8. HRMS (ESI): calc'd for $\mathrm{C}_{19} \mathrm{H}_{21} \mathrm{~N}_{2} \mathrm{O}_{3}\left([\mathrm{M}+\mathrm{H}]^{+}\right) 325.1546$. Found: 325.1539 .

\section{2-o-Tolyl-pyrrolo[3,2-c]pyridine-1-carboxylic acid tert-butyl ester (27d)}

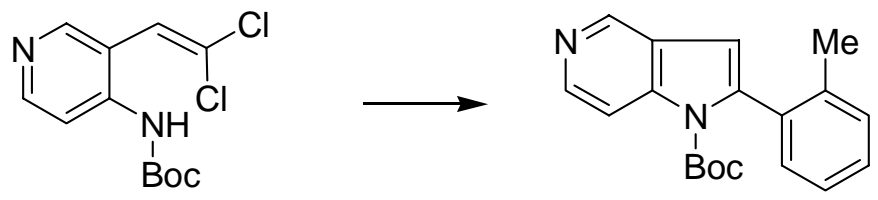

The general procedure for the tandem coupling was followed using 10 (58 $\mathrm{mg}, 0.2$ mmol), o-tolylboronic acid (32.6 mg, $0.24 \mathrm{mmol}), \mathrm{K}_{3} \mathrm{PO}_{4} \cdot \mathrm{H}_{2} \mathrm{O}(230 \mathrm{mg}, 1 \mathrm{mmol})$, $\mathrm{Pd}(\mathrm{OAc})_{2}(2.3 \mathrm{mg}, 0.01 \mathrm{mmol})$, and X-Phos $(9.5 \mathrm{mg}, 0.02 \mathrm{mmol})$. The reaction mixture was heated in $\mathrm{PhMe}(2 \mathrm{~mL})$ at $100{ }^{\circ} \mathrm{C}$ for $2.5 \mathrm{~h}$. The crude material after workup was purified using chromatography eluting with 33\% EtOAc in hexanes to give the desired product as a white solid (46.2 $\mathrm{mg}, 75 \%$ ). $\mathrm{mp} \mathrm{104-105}{ }^{\circ} \mathrm{C}$. IR (neat, $\mathrm{cm}^{-1}$ ): 2979, 2930, 1739, 1457, 1340, 1228, 1154. ${ }^{1} \mathrm{H}$ NMR (400 MHz, $\left.\mathrm{CDCl}_{3}\right) \delta 8.87(1 \mathrm{H}, \mathrm{s}), 8.49$ (1H, d, J $=5.9 \mathrm{~Hz}), 8.11(1 \mathrm{H}, \mathrm{d}, J=5.7 \mathrm{~Hz}), 7.34-7.24(4 \mathrm{H}, \mathrm{m}), 6.52(1 \mathrm{H}, \mathrm{d}, J=0.7 \mathrm{~Hz}), 2.17(3 \mathrm{H}$, s), $1.29(9 \mathrm{H}, \mathrm{s}) .{ }^{13} \mathrm{C} \mathrm{NMR}\left(100 \mathrm{MHz}, \mathrm{CDCl}_{3}\right) \delta 149.6,144.1,143.4,141.4,140.6,137.4$, 
134.4, 129.9, 129.7, 128.8, 125.9, 125.6, 110.6, 107.7, 84.3, 27.6, 20.1. HRMS (ESI): calc'd for $\mathrm{C}_{19} \mathrm{H}_{21} \mathrm{~N}_{2} \mathrm{O}_{2}\left([\mathrm{M}+\mathrm{H}]^{+}\right)$309.1597.1441. Found: 309.1596 .

\section{Thiophen-3-yl-pyrrolo[2,3-c]pyridine-1-carboxylic acid tert-butyl ester (27e)}

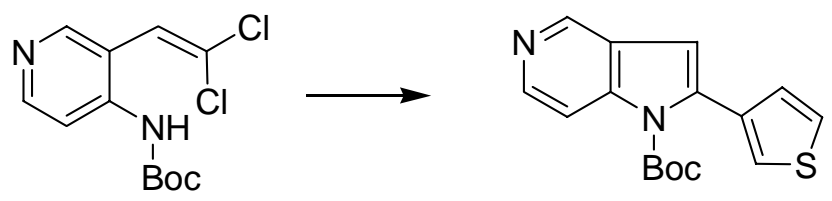

The general procedure for the tandem coupling was followed using 10 (58 $\mathrm{mg}, 0.2$ mmol), 3-thienoboronic acid (31 mg, $0.24 \mathrm{mmol}), \mathrm{K}_{3} \mathrm{PO}_{4} \cdot \mathrm{H}_{2} \mathrm{O}(230 \mathrm{mg}, 1 \mathrm{mmol})$, $\mathrm{Pd}(\mathrm{OAc})_{2}(2.3 \mathrm{mg}, 0.01 \mathrm{mmol})$, and $\mathrm{S}-\mathrm{Phos}(8.2 \mathrm{mg}, 0.02 \mathrm{mmol})$. The reaction mixture was heated in $\mathrm{PhMe}(2 \mathrm{~mL})$ at $100{ }^{\circ} \mathrm{C}$ for $15 \mathrm{~h}$. The crude material after workup was purified using chromatography eluting with 33\% EtOAc in hexanes to give the desired product as a yellow oil (48.3 $\mathrm{mg}, 80 \%) . \mathrm{mp} \mathrm{112-113}{ }^{\circ} \mathrm{C}$. IR (neat, $\mathrm{cm}^{-1}$ ): 2980, 1739, 1459, 1338, 1152. ${ }^{1} \mathrm{H}$ NMR $\left(400 \mathrm{MHz}, \mathrm{CDCl}_{3}\right) \delta 8.86(1 \mathrm{H}, \mathrm{s}), 8.48(1 \mathrm{H}, \mathrm{d}, J=5.9 \mathrm{~Hz})$, $8.03(1 \mathrm{H}, \mathrm{d}, J=5.7 \mathrm{~Hz}), 7.38-7.34(2 \mathrm{H}, \mathrm{m}), 7.14(1 \mathrm{H}, \mathrm{dd}, J=4.8,1.3 \mathrm{~Hz}), 6.63(1 \mathrm{H}, \mathrm{d}, J$ $=0.7 \mathrm{~Hz}), 1.43(9 \mathrm{H}, \mathrm{s}) .{ }^{13} \mathrm{C} \mathrm{NMR}\left(100 \mathrm{MHz}, \mathrm{CDCl}_{3}\right) \delta 149.6,144.3,143.4,141.7$, 136.5, 134.0, 129.0, 125.6, 125.1, 124.0, 110.4, 108.3, 84.8, 27.8. HRMS (ESI): calc'd for $\mathrm{C}_{16} \mathrm{H}_{17} \mathrm{~N}_{2} \mathrm{O}_{2} \mathrm{~S}\left([\mathrm{M}+\mathrm{H}]^{+}\right)$301.1005. Found: 301.1012 .

\section{[3-(2-Chloro-2-thiophen-3-yl-vinyl)-pyridin-4-yl]-carbamic acid tert-butyl ester (40)}



The general procedure for the tandem coupling was followed using 10 (58 $\mathrm{mg}, 0.2$ mmol), 3-thienoboronic acid (31mg, $0.24 \mathrm{mmol}), \mathrm{K}_{3} \mathrm{PO}_{4} \cdot \mathrm{H}_{2} \mathrm{O}(230 \mathrm{mg}, 1 \mathrm{mmol})$, $\mathrm{Pd}(\mathrm{OAc})_{2}(2.3 \mathrm{mg}, 0.01 \mathrm{mmol})$, and X-Phos $(9.5 \mathrm{mg}, 0.02 \mathrm{mmol})$. The reaction mixture was heated in $\mathrm{PhMe}(2 \mathrm{~mL})$ at $100{ }^{\circ} \mathrm{C}$ for $2.5 \mathrm{~h}$. The crude material after workup was purified using chromatography eluting with 33\% EtOAc in hexanes to give the desired product as a yellow oil (50 mg, 74\%). mp 126-128 ${ }^{\circ} \mathrm{C}$. IR (neat, $\mathrm{cm}^{-1}$ ): 3421, 2976, 1731, 
1574, 1508, 1246, 1154. ${ }^{1} \mathrm{H}$ NMR $\left(400 \mathrm{MHz}, \mathrm{CDCl}_{3}\right) \delta 8.52(1 \mathrm{H}, \mathrm{s}), 8.44(1 \mathrm{H}, \mathrm{d}, J=5.6$ $\mathrm{Hz}), 8.08(1 \mathrm{H}, \mathrm{d}, J=5.9 \mathrm{~Hz}), 7.70(1 \mathrm{H}, \mathrm{dd}, J=2.4,1.7 \mathrm{~Hz}), 7.44-7.40(2 \mathrm{H}, \mathrm{m}), 6.89(1 \mathrm{H}$, s), $6.64(1 \mathrm{H}, \mathrm{br}), 1.52(9 \mathrm{H}, \mathrm{s}) .{ }^{13} \mathrm{C} \mathrm{NMR}\left(100 \mathrm{MHz}, \mathrm{CDCl}_{3}\right) \delta 152.0,150.8,150.1,143.5$, 139.2, 134.1, 127.2, 125.6, 125.2, 119.7, 116.6, 112.6, 82.2, 28.4. HRMS (ESI): calc'd for $\mathrm{C}_{16} \mathrm{H}_{18} \mathrm{~N}_{2} \mathrm{O}_{2} \mathrm{SCl}\left([\mathrm{M}+\mathrm{H}]^{+}\right)$337.0772. Found: 337.0784 .

\section{Thiophen-3-yl-pyrrolo[2,3-c]pyridine-1-carboxylic acid tert-butyl ester (27e)}

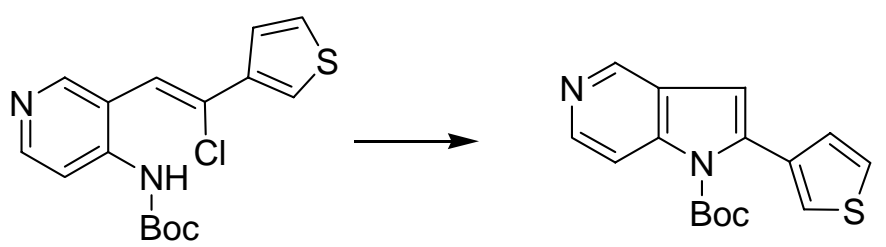

The general procedure for the tandem coupling was followed using 10 (40 mg, 0.12 $\mathrm{mmol}), \mathrm{K}_{3} \mathrm{PO}_{4} \cdot \mathrm{H}_{2} \mathrm{O}$ (110 mg, $\left.0.48 \mathrm{mmol}\right), \mathrm{Pd}(\mathrm{OAc})_{2}(1.3 \mathrm{mg}, 0.006 \mathrm{mmol})$, and S-Phos (4.9 $\mathrm{mg}, 0.012 \mathrm{mmol})$. The reaction mixture was heated in $\mathrm{PhMe}(1.5 \mathrm{~mL})$ at $100{ }^{\circ} \mathrm{C}$ for $5 \mathrm{~h}$. The crude material after workup was purified using chromatography eluting with $33 \%$ EtOAc in hexanes to give the desired product as a yellow oil (29 $\mathrm{mg}, 80 \%)$.

\section{5-Oxy-2-phenyl-pyrrolo[3,2-c]pyridine-1-carboxylic acid tert-butyl ester (30a)}



The general procedure for the tandem coupling was followed using 29 (52 $\mathrm{mg}, 0.17$ mmol), phenylboronic acid (32 mg, $0.26 \mathrm{mmol}), \mathrm{K}_{3} \mathrm{PO}_{4} \cdot \mathrm{H}_{2} \mathrm{O}(119 \mathrm{mg}, 0.51 \mathrm{mmol})$, $\mathrm{Pd}(\mathrm{OAc})_{2}(1.9 \mathrm{mg}, 0.0084 \mathrm{mmol})$, and S-Phos $(7.1 \mathrm{mg}, 0.0168 \mathrm{mmol})$. The reaction mixture was heated in $\mathrm{PhMe}(3 \mathrm{~mL})$ at $100{ }^{\circ} \mathrm{C}$ for $1.5 \mathrm{~h}$, then the reaction mixture was diluted in $\mathrm{MeOH}$ and the solids filtered off. The crude material was purified using chromatography eluting with $10 \% \rightarrow 15 \% \mathrm{MeOH} / \mathrm{EtOAc}$ to give the product as a yellow solid (31 mg, 65\%). mp 150-152 ${ }^{\circ} \mathrm{C}$. IR (neat, $\mathrm{cm}^{-1}$ ): 1734, 1449, 1333, 1130. ${ }^{1} \mathrm{H}$ NMR 
$\left(400 \mathrm{MHz}, \mathrm{CDCl}_{3}\right) \delta 8.53(1 \mathrm{H}, \mathrm{d}, J=1.8 \mathrm{~Hz}), 8.18(1 \mathrm{H}, \mathrm{dd}, J=7.3,1.8 \mathrm{~Hz}), 8.08(1 \mathrm{H}, \mathrm{d}$, $J=7.0 \mathrm{~Hz}), 7.47-7.38(5 \mathrm{H}, \mathrm{m}), 6.51(1 \mathrm{H}, \mathrm{s}), 1.30(9 \mathrm{H}, \mathrm{s}) .{ }^{13} \mathrm{C} \mathrm{NMR}\left(100 \mathrm{MHz}, \mathrm{CDCl}_{3}\right) \delta$ $149.1,144.9,135.5,134.3,133.2,131.7,129.0,128.9,128.2,127.1,112.5,106.6,85.6$, 27.6. HRMS (EI): calc'd for $\mathrm{C}_{18} \mathrm{H}_{18} \mathrm{~N}_{2} \mathrm{O}_{3}\left([\mathrm{M}]^{+}\right)$310.1317. Found: 310.1313 .

\section{2-(4-Methoxyphenyl)-5-oxy-pyrrolo[3,2-c]pyridine-1-carboxylic acid tert-butyl ester} (30b)

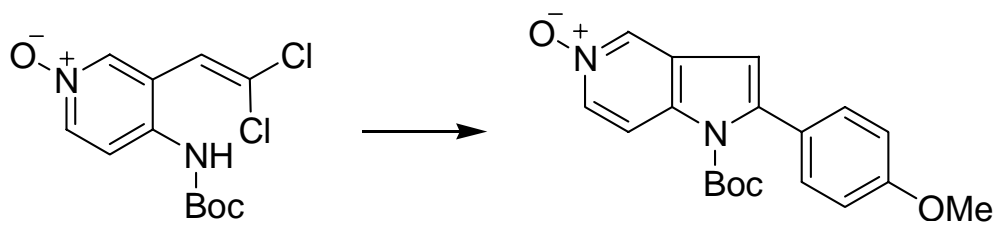

The general procedure for the tandem coupling was followed using 29 (52 $\mathrm{mg}, 0.17$ mmol), 4-methoxyphenyl (40 mg, $0.26 \mathrm{mmol}), \mathrm{K}_{3} \mathrm{PO}_{4} \cdot \mathrm{H}_{2} \mathrm{O}(117 \mathrm{mg}, 0.51 \mathrm{mmol})$, $\mathrm{Pd}(\mathrm{OAc})_{2}(1.9 \mathrm{mg}, 0.0084 \mathrm{mmol})$, and S-Phos $(7.0 \mathrm{mg}, 0.0168 \mathrm{mmol})$. The reaction mixture was heated in $\mathrm{PhMe}(3.4 \mathrm{~mL})$ at $100{ }^{\circ} \mathrm{C}$ for $1.5 \mathrm{~h}$, then the reaction mixture was diluted in $\mathrm{MeOH}$ and the solids filtered off. The crude material was purified using chromatography eluting with $5 \% \rightarrow 15 \% \mathrm{MeOH} / \mathrm{EtOAc}$ to give the product as a colourless gum (44 mg, 76\%). IR (neat, $\mathrm{cm}^{-1}$ ): 2924, 1739, 1447, 1335, 1132. ${ }^{1} \mathrm{H}$ NMR (400 MHz, $\left.\mathrm{CDCl}_{3}\right) \delta 8.51(1 \mathrm{H}, \mathrm{d}, J=1.3 \mathrm{~Hz}), 8.17(1 \mathrm{H}, \mathrm{dd}, J=7.0,1.7 \mathrm{~Hz}), 8.04(1 \mathrm{H}, \mathrm{d}, J=7.3$ $\mathrm{Hz}), 7.33\left(2 \mathrm{H}, \mathrm{dt}, J^{\mathrm{d}}=8.8 \mathrm{~Hz}, J^{\mathrm{t}}=2.9 \mathrm{~Hz}\right), 6.97\left(2 \mathrm{H}, \mathrm{dt}, J^{\mathrm{d}}=8.8 \mathrm{~Hz}, J^{\mathrm{d}}=2.9 \mathrm{~Hz}\right), 6.46$ $(1 \mathrm{H}, \mathrm{d}, J=0.4 \mathrm{~Hz}), 3.07(3 \mathrm{H}, \mathrm{s}), 1.37(9 \mathrm{H}, \mathrm{s}) .{ }^{13} \mathrm{C} \mathrm{NMR}\left(100 \mathrm{MHz}, \mathrm{CDCl}_{3}\right) \delta 160.3$, 149.2 , 144.9, 135.2, 134.3, 131.5, 130.3, 127.1, 125.4, 113.7, 112.4, 106.3, 85.6, 55.6, 27.7. HRMS (ESI): calc'd for $\mathrm{C}_{19} \mathrm{H}_{21} \mathrm{~N}_{2} \mathrm{O}_{4}\left([\mathrm{M}+\mathrm{H}]^{+}\right)$341.1495. Found: 341.1508 .

\section{2-Phenyl-1H-pyrrolo[3,2-b]pyridine 4-oxide (35a)}<smiles>O=C(O)Nc1ccc[n+]([O-])c1C=C(Cl)Cl</smiles><smiles>[O-][n+]1cccc2[nH]c(-c3ccccc3)cc21</smiles> 
The general procedure for the tandem coupling was followed using 34 (58 $\mathrm{mg}, 0.17$ mmol), phenylboronic acid (23 mg, $0.19 \mathrm{mmol}), \mathrm{K}_{3} \mathrm{PO}_{4} \cdot \mathrm{H}_{2} \mathrm{O}(114 \mathrm{mg}, 0.50 \mathrm{mmol})$, $\mathrm{Pd}(\mathrm{OAc})_{2}(1.9 \mathrm{mg}, 0.0084 \mathrm{mmol})$, and S-Phos $(7.0 \mathrm{mg}, 0.0168 \mathrm{mmol})$. The reaction mixture was heated in $\mathrm{PhMe}(3.5 \mathrm{~mL})$ at $100{ }^{\circ} \mathrm{C}$ for $2 \mathrm{~h}$, then the reaction mixture was diluted in $\mathrm{MeOH}$, and the solids filtered off. The crude material was purified using chromatography eluting with $10 \% \rightarrow 20 \% \mathrm{MeOH}$ in EtOAc to give the product as a yellow solid (25 mg, 70\%). mp $150{ }^{\circ} \mathrm{C}$ (decomp.). IR (neat, $\mathrm{cm}^{-1}$ ): 3317, 1452, 1130. ${ }^{1} \mathrm{H}$ NMR (400 MHz, CD 3 OD) $\delta 8.10(1 \mathrm{H}, \mathrm{dd}, J=6.2,0.6 \mathrm{~Hz}), 7.84-7.79(2 \mathrm{H}, \mathrm{m}), 7.64(1 \mathrm{H}$, $\left.\mathrm{dt}, J^{\mathrm{d}}=8.3 \mathrm{~Hz}, J^{\mathrm{t}}=0.9 \mathrm{~Hz}\right), 7.47-7.42(2 \mathrm{H}, \mathrm{m}), 7.37(1 \mathrm{H}, \mathrm{tt}, J=6.4,1.3 \mathrm{~Hz}), 7.16(1 \mathrm{H}$, $\mathrm{dd}, J=8.1,6.1 \mathrm{~Hz}), 7.10(1 \mathrm{H}, \mathrm{d}, J=0.9 \mathrm{~Hz}) .{ }^{13} \mathrm{C} \mathrm{NMR}\left(100 \mathrm{MHz}, \mathrm{CD}_{3} \mathrm{OD}\right) \delta 145.1$, 138.6, 135.6, 132.8, 131.9, 130.8, 130.5, 127.3, 118.7, 115.8, 93.7. HRMS (ESI): calc'd for $\mathrm{C}_{13} \mathrm{H}_{11} \mathrm{~N}_{2} \mathrm{O}\left([\mathrm{M}+\mathrm{H}]^{+}\right)$211.0865. Found: 211.0873 .

\section{2-(4-Methoxyphenyl)-1H-pyrrolo[3,2-b]pyridine 4-oxide (35b)}
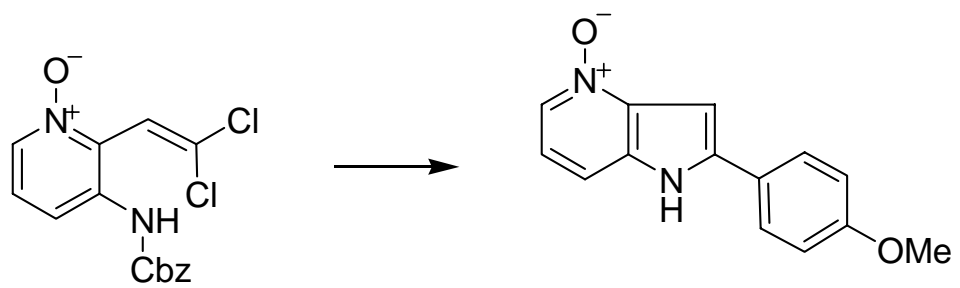

The general procedure for the tandem coupling was followed using 34 (58 mg, 0.17 mmol), 4-methoxyphenylboronic acid (32 mg, $0.21 \mathrm{mmol}), \mathrm{K}_{3} \mathrm{PO}_{4} \cdot \mathrm{H}_{2} \mathrm{O}(114 \mathrm{mg}, 0.50$ $\mathrm{mmol}), \mathrm{Pd}(\mathrm{OAc})_{2}(1.9 \mathrm{mg}, 0.0084 \mathrm{mmol})$, and S-Phos $(7.0 \mathrm{mg}, 0.0168 \mathrm{mmol})$. The reaction mixture was heated in $\mathrm{PhMe}(3.4 \mathrm{~mL})$ at $100{ }^{\circ} \mathrm{C}$ for $1 \mathrm{~h}$, then the reaction mixture was diluted in $\mathrm{MeOH}$, and the solids filtered off. The crude material was purified using chromatography eluting with $10 \% \rightarrow 30 \% \mathrm{MeOH} / \mathrm{EtOAc}$ to give the product as a pale brown solid (40 mg, 98\%). mp $270{ }^{\circ} \mathrm{C}$ (decomp.). IR (neat, $\mathrm{cm}^{-1}$ ): 3413, 1650, 1011. ${ }^{1} \mathrm{H}$ NMR (500 MHz, DMSO) $\delta 12.32(1 \mathrm{H}, \mathrm{s}), 8.0(1 \mathrm{H}, \mathrm{d}, J=6.2 \mathrm{~Hz}), 7.91(2 \mathrm{H}, \mathrm{d}, J=8.8$ $\mathrm{Hz}), 7.40(1 \mathrm{H}, \mathrm{d}, J=8.2 \mathrm{~Hz}), 7.08-7.03(4 \mathrm{H}, \mathrm{m}), 3.37(3 \mathrm{H}, \mathrm{s}) .{ }^{13} \mathrm{C}$ NMR $(125 \mathrm{MHz}$, DMSO) $\delta 159.7,140.4,137.1,133.1,130.3,127.1,123.1,117.3,114.5,109.6,92.0,55.3$. HRMS (ESI): calc'd for $\mathrm{C}_{14} \mathrm{H}_{13} \mathrm{~N}_{2} \mathrm{O}_{2}\left([\mathrm{M}+\mathrm{H}]^{+}\right)$241.0971. Found: 241.0977. 


\section{2-Phenyl-1H-pyrrolo[3,2-b]pyridine (36)}

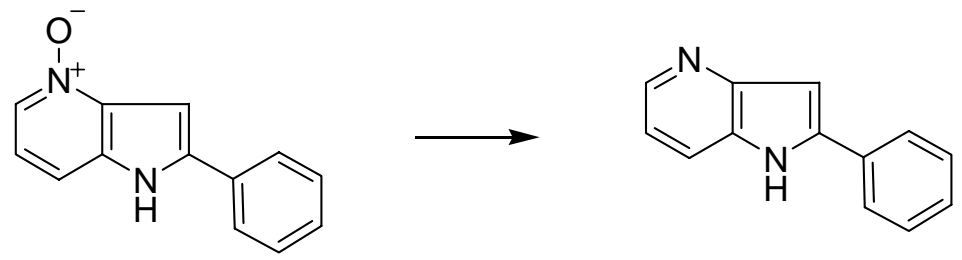

Phosphorus trichloride $(0.02 \mathrm{~mL}, 0.22 \mathrm{mmol})$ was added dropwise to a stirred solution of $35 \mathrm{a}(12 \mathrm{mg}, 0.038 \mathrm{mmol})$ in $\mathrm{CHCl}_{3}(1 \mathrm{~mL})$. The reaction mixture was heated at $80{ }^{\circ} \mathrm{C}$ for $7 \mathrm{~h}$. Ice was added to the reaction mixture, and then neutralized to $\mathrm{pH} 10$ using saturated $\mathrm{NaHCO}_{3}$ solution. After stirring for $30 \mathrm{~min}$, the mixture was extracted using $\mathrm{CHCl}_{3}(3 \times 15 \mathrm{~mL})$. The combined organic layers were washed with brine, dried over $\mathrm{MgSO}_{4}$, filtered, and concentrated in vacuo. The crude material was purified using chromatography eluting with $100 \% \mathrm{EtOAc} \rightarrow 10 \% \mathrm{MeOH} / \mathrm{EtOAc}$ to give the product as a white solid (6 mg, $0.03 \mathrm{mmol}, 80 \%$ ). mp 250-252 ${ }^{\circ} \mathrm{C}$. IR (neat, $\mathrm{cm}^{-1}$ ): $3439,1642 .{ }^{1} \mathrm{H}$ NMR (400 MHz, CD $\mathrm{OD}) \delta 7.23(1 \mathrm{H}, \mathrm{s}), 6.58(1 \mathrm{H}, \mathrm{d}, J=5.7 \mathrm{~Hz}), 6.29(2 \mathrm{H}, \mathrm{d}, J=7.7$ $\mathrm{Hz}), 5.93(2 \mathrm{H}, \mathrm{t}, J=7.5 \mathrm{~Hz}), 5.89(1 \mathrm{H}, \mathrm{d}, J=5.9 \mathrm{~Hz}), 5.82(1 \mathrm{H}, \mathrm{t}, J=7.3 \mathrm{~Hz}), 5.45(1 \mathrm{H}$, s). ${ }^{13} \mathrm{C}$ NMR $\left(100 \mathrm{MHz}, \mathrm{CD}_{3} \mathrm{OD}\right) \delta 143.5,142.8,141.9,140.5,133.2,130.3,129.5$, 127.9, 126.8, 108.1, 99.1, 99.1. HRMS (ESI): calc'd for $\mathrm{C}_{13} \mathrm{H}_{10} \mathrm{~N}_{2}\left([\mathrm{M}]^{+}\right)$194.0844. Found: 194.0852.

\section{3-Iodo-1-methyl-2-phenyl-1H-pyrrolo[2,3-b]pyridine (22)}
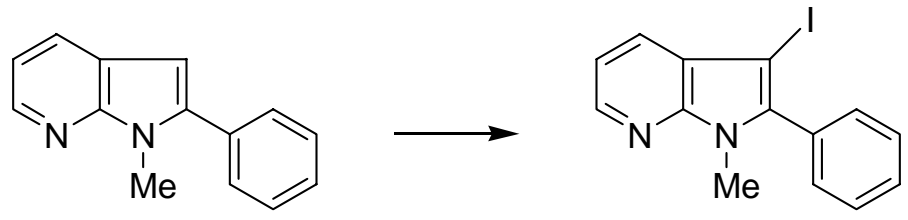

To a solution of 6a $(34.5 \mathrm{mg}, 0.166 \mathrm{mmol})$ in DCM $(1 \mathrm{~mL})$ at $0{ }^{\circ} \mathrm{C}$ was added $N$ iodosuccimide (56 mg, $0.248 \mathrm{mmol}$ ). The mixture was stirred for $30 \mathrm{~min}$, warmed to rt, and stirred for an additional $2 \mathrm{~h}$. The mixture was diluted with $\mathrm{Et}_{2} \mathrm{O}(10 \mathrm{~mL})$, and quenched with KI solution, washed with $\mathrm{Na}_{2} \mathrm{~S}_{2} \mathrm{O}_{3}, \mathrm{NaHCO}_{3}$, brine, and dried over 
$\mathrm{Na}_{2} \mathrm{SO}_{4}$. The crude material was purified by flash chromatography (20\% EtOAc in hexanes) to afford a white crystalline solid $(53.2 \mathrm{mg}, 96 \%)$. mp $84-85^{\circ} \mathrm{C}$. IR (neat, $\mathrm{cm}^{-1}$ ): 3051, 1592, 1566, 1480, 1448, 1402, 1309, 1111. ${ }^{1} \mathrm{H}$ NMR (400 MHz, $\left.\mathrm{CDCl}_{3}\right) \delta 8.37$ $(1 \mathrm{H}, \mathrm{dd}, J=4.6,1.8 \mathrm{~Hz}), 7.75(1 \mathrm{H}, \mathrm{dd}, J=7.9,1.5 \mathrm{~Hz}), 7.56-7.46(5 \mathrm{H}, \mathrm{m}), 7.16(1 \mathrm{H}, \mathrm{dd}$, $J=7.9,4.8 \mathrm{~Hz}$ ), $3.79(3 \mathrm{H}, \mathrm{s}) .{ }^{13} \mathrm{C} \mathrm{NMR}\left(100 \mathrm{MHz}, \mathrm{CDCl}_{3}\right) \delta 148.7,144.0,142.1,131.1$, 130.7, 129.2, 129.1, 128.5, 123.7, 116.9, 56.5, 30.5. HRMS (ESI): calc'd for $\mathrm{C}_{14} \mathrm{H}_{12} \mathrm{~N}_{2} \mathrm{I}$ $\left([\mathrm{M}+\mathrm{H}]^{+}\right)$335.0039. Found: 335.0044 .

\section{3-Iodo-1-methyl-2-phenyl-1H-pyrrolo[2,3-b]pyridine (21)}



To a 10-mL microwave tube was charged $22(33.3 \mathrm{mg}, 0.1 \mathrm{~mL})$, the acrylamide 23 (24.7 mg, $0.1 \mathrm{mmol}), \mathrm{Bu} 4 \mathrm{NCl}(28 \mathrm{mg}, 0.1 \mathrm{mmol}), \mathrm{Na}_{2} \mathrm{CO}_{3}(0.1 \mathrm{mmol})$, and $\mathrm{Pd}(\mathrm{OAc})_{2}$ (1.2 $\mathrm{mg}, 0.005 \mathrm{mmol})$. The tube was sealed, and purged with argon for $5 \mathrm{~min}$ before addition of anhydrous DMF $(0.5 \mathrm{~mL})$, and purged for another 5 min while stirring. The reaction vessel was subjected to controlled microwave radiation at $200{ }^{\circ} \mathrm{C}$ for $5 \mathrm{~min}$. To the mixture was added $\mathrm{H}_{2} \mathrm{O}(5 \mathrm{~mL})$, and the resulting mixture was extracted with EtOAc $(3 \mathrm{~mL})$ and $\mathrm{Et}_{2} \mathrm{O}(2 \times 5 \mathrm{~mL})$. The combined organic layers were washed with $\mathrm{H}_{2} \mathrm{O}(2 \times 5$ $\mathrm{mL}), \mathrm{NaHCO}_{3}(5 \mathrm{~mL})$, brine $(5 \mathrm{~mL})$, and dried over $\mathrm{Na}_{2} \mathrm{SO}_{4}$. The crude material was purified by flash chromatography ( $75 \%$ EtoAc in hexanes) to afford a foamy solid (40.8 mg, 90\%). IR (neat, $\mathrm{cm}^{-1}$ ): 2934, 1644, 1591, 1517, 1454, 1270, 1113. ${ }^{1} \mathrm{H}$ NMR (400 $\left.\mathrm{MHz}, \mathrm{CDCl}_{3}\right) \delta 8.43(1 \mathrm{H}, \mathrm{dd}, J=4.6,1.5 \mathrm{~Hz}), 8.24(1 \mathrm{H}, \mathrm{br}), 7.77(1 \mathrm{H}, \mathrm{d}, J=15.4 \mathrm{~Hz})$, 7.56-7.50 (3H, m), 7.46-7.43 (2H, m), 7.24 (1H, dd, $J=7.7,4.6 \mathrm{~Hz}), 6.87(1 \mathrm{H}, \mathrm{d}, J=$ $15.4 \mathrm{~Hz}), 6.64(2 \mathrm{H}, \mathrm{s}), 4.73(2 \mathrm{H}, \mathrm{br}), 3.86-3.81(8 \mathrm{H}, \mathrm{m}), 3.76(3 \mathrm{H}, \mathrm{s}), 2.85(2 \mathrm{H}, \mathrm{br}) .{ }^{13} \mathrm{C}$ NMR $\left(100 \mathrm{MHz}, \mathrm{CDCl}_{3}\right.$ ) (two rotamers) $\delta 167.0,149.1,148.0,144.6,143.8,136.2$, $130.9,130.1,129.5,129.0,128.4,126.2,126.2,126.0,124.6,118.7,117.3,114.0,113.7$, 111.8, 111.4, 109.7, 109.3, 56.2, 47.3, 44.6, 43.9, 40.2, 30.0, 29.4, 28.4. HRMS (ESI): calc'd for $\mathrm{C}_{28} \mathrm{H}_{28} \mathrm{~N}_{3} \mathrm{O}_{3}\left([\mathrm{M}+\mathrm{H}]^{+}\right)$454.2125. Found: 454.2116. 


\section{Preparation of Thienopyrroles}

\section{5-Phenylthieno[2,3-b]pyrrole-6-carboxylic acid tert-butyl ester (38a)}

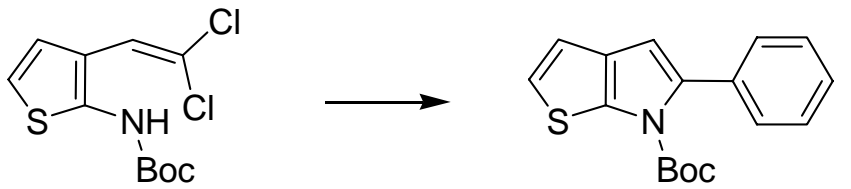

The general procedure for the tandem coupling was followed using 16 (72.7 $\mathrm{mg}$, $0.247 \mathrm{mmol}$ ), phenylboronic acid (36 mg, $0.30 \mathrm{mmol}), \mathrm{K}_{3} \mathrm{PO}_{4} \cdot \mathrm{H}_{2} \mathrm{O}$ (284 mg, 1.235 $\mathrm{mmol}), \mathrm{Pd}(\mathrm{OAc})_{2}(1.66 \mathrm{mg}, 0.0074 \mathrm{mmol})$, and S-Phos $(6.1 \mathrm{mg}, 0.0148 \mathrm{mmol})$. The reaction mixture was heated in PhMe $(2.5 \mathrm{~mL})$ at $100{ }^{\circ} \mathrm{C}$ for $15 \mathrm{~h}$. The crude material was purified using chromatography eluting with 3.5\% EtOAc in hexanes to give the product as a white solid (56 mg, 76\%). $\mathrm{mp} \mathrm{88-89}{ }^{\circ} \mathrm{C}$. IR (neat, $\mathrm{cm}^{-1}$ ): 2979, 2928, 1754, 1726, 1463, 1367, 1315, 1164, 1140, 1121, 1044. ${ }^{1} \mathrm{H}$ NMR (400 MHz, $\left.\mathrm{CDCl}_{3}\right) \delta 7.43-$ $7.30(5 \mathrm{H}, \mathrm{m}), 7.01(1 \mathrm{H}, \mathrm{AB}, J=5.5 \mathrm{~Hz}), 6.99(1 \mathrm{H}, \mathrm{AB}, J=5.5 \mathrm{~Hz}), 6.47(1 \mathrm{H}, \mathrm{s}), 1.42$ (9H, s). $\left.{ }^{13} \mathrm{C} \mathrm{NMR} \mathrm{(100} \mathrm{MHz,} \mathrm{CDCl}_{3}\right) \delta 149.1,140.0,136.2,134.4,130.6,129.6,127.8$, 127.8, 121.7, 117.4, 108.6, 84.7, 28.0. HRMS (ESI): calc'd for $\mathrm{C}_{17} \mathrm{H}_{17} \mathrm{NO}_{2} \mathrm{NaS}$ $\left([\mathrm{M}+\mathrm{Na}]^{+}\right)$322.0872. Found: 322.0874 .

\section{5-Styrylthieno[2,3-b]pyrrole-6-carboxylic acid tert-butyl ester (38c)}

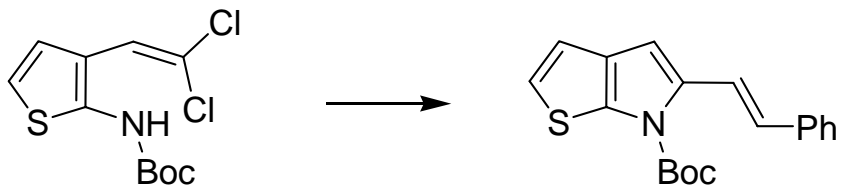

The general procedure for the tandem coupling was followed using 16 (29.5 $\mathrm{mg}, 0.10$ mmol), styrenylboronic acid (17.8 $\mathrm{mg}, 0.12 \mathrm{mmol}), \mathrm{K}_{3} \mathrm{PO}_{4} \cdot \mathrm{H}_{2} \mathrm{O}$ (115 mg, $\left.0.5 \mathrm{mmol}\right)$, $\mathrm{Pd}(\mathrm{OAc})_{2}(2.3 \mathrm{mg}, 0.01 \mathrm{mmol})$, and DavePhos $(7.87 \mathrm{mg}, 0.02 \mathrm{mmol})$. The reaction mixture was heated in PhMe $(1 \mathrm{~mL})$ at $100{ }^{\circ} \mathrm{C}$ for $5 \mathrm{~h}$. The crude material was purified using chromatography eluting with $3.5 \%$ EtOAc in hexanes to give the product as a 
yellowish oil (23.1 mg, 71\%). IR (neat, $\mathrm{cm}^{-1}$ ): 3081, 2979, 1742, 1403, 1366, 1326, 1165 , 1113. ${ }^{1} \mathrm{H}$ NMR (400 MHz, $\left.\mathrm{CDCl}_{3}\right) \delta 7.84(1 \mathrm{H}, \mathrm{d}, J=16.3 \mathrm{~Hz}), 7.50\left(2 \mathrm{H}, \mathrm{dm}, J^{\mathrm{d}}=8.3\right.$ $\mathrm{Hz}), 7.33(2 \mathrm{H}, \mathrm{t}, J=7.7 \mathrm{~Hz}), 7.23(1 \mathrm{H}, \mathrm{tt}, J=7.5,1.2 \mathrm{~Hz}), 6.98(1 \mathrm{H}, \mathrm{AB}, J=5.3 \mathrm{~Hz})$, $6.96(1 \mathrm{H}, \mathrm{AB}, J=5.3 \mathrm{~Hz}), 6.96(1 \mathrm{H}, \mathrm{d}, J=15.8 \mathrm{~Hz}), 6.83(1 \mathrm{H}, \mathrm{d}, J=0.7 \mathrm{~Hz}), 1.70(9 \mathrm{H}$, s). ${ }^{13} \mathrm{C} \mathrm{NMR}\left(100 \mathrm{MHz}, \mathrm{CDCl}_{3}\right) \delta 149.2,139.5,137.6,136.0,130.9,128.8,128.6,127.7$, 126.7, 122.0, 120.1, 117.3, 104.4, 85.3, 28.4. HRMS (ESI): calc'd for $\mathrm{C}_{19} \mathrm{H}_{20} \mathrm{NO}_{2} \mathrm{~S}$ $\left([\mathrm{M}+\mathrm{H}]^{+}\right)$326.1209. Found: 326. 1225.

[3-(2-Chloro-2-o-tolylvinyl)-thiophen-2-yl]-carbamic acid tert-butyl ester (41) and 5o-Tolylthieno[2,3-b]pyrrole-6-carboxylic acid tert-butyl ester (38d)

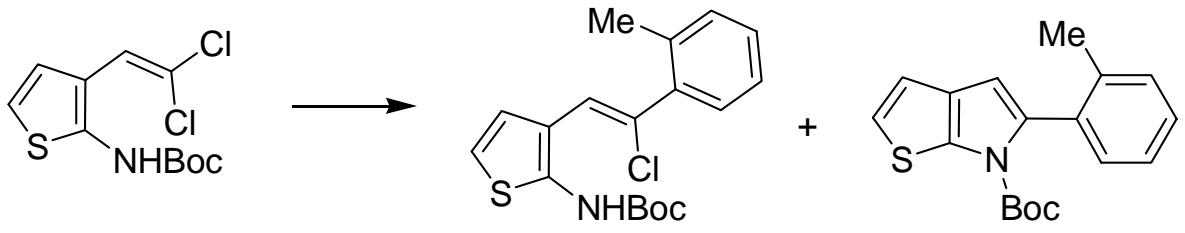

The general procedure for the tandem coupling was followed using 16 (73.6 mg, 0.25 mmol), o-tolylboronic acid (51 mg, $0.375 \mathrm{mmol}), \mathrm{K}_{3} \mathrm{PO}_{4} \cdot \mathrm{H}_{2} \mathrm{O}(288 \mathrm{mg}, 1.25 \mathrm{mmol})$, $\mathrm{Pd}(\mathrm{OAc})_{2}(2.9 \mathrm{mg}, 0.0125 \mathrm{mmol})$, and $\mathrm{S}-\mathrm{Phos}(10.3 \mathrm{mg}, 0.015 \mathrm{mmol})$. The reaction mixture was heated in $\mathrm{PhMe}(2.5 \mathrm{~mL})$ at $100{ }^{\circ} \mathrm{C}$ for $18 \mathrm{~h}$. The crude material was separated using chromatography eluting with $3.5 \% \rightarrow 5 \%$ EtOAc in hexanes to give 38d (less polar, $4.4 \mathrm{mg}, 6 \%$ ) and 41 (more polar, $52.4 \mathrm{mg}, 60 \%$ ) both as oils.

38d: IR (neat, $\mathrm{cm}^{-1}$ ): 2978, 2916, 1748, 1726, 1465, 1395, 1312, 1112, 1019. ${ }^{1} \mathrm{H}$ NMR (400 MHz, $\left.\mathrm{CDCl}_{3}\right) \delta$ 7.30-7.19 (4H, m), $7.03(1 \mathrm{H}, \mathrm{AB}, J=5.3 \mathrm{~Hz}), 7.01(1 \mathrm{H}, \mathrm{AB}$, $J=5.3 \mathrm{~Hz}), 6.39(1 \mathrm{H}, \mathrm{s}), 2.20(3 \mathrm{H}, \mathrm{s}), 1.32(9 \mathrm{H}, \mathrm{s}) \cdot{ }^{13} \mathrm{C} \mathrm{NMR}\left(100 \mathrm{MHz}, \mathrm{CDCl}_{3}\right) \delta$ $149.1,138.6,138.3,135.2,134.8,130.6,129.5,128.5,125.4,121.5,117.4,108.0,84.2$, 27.8, 20.3. HRMS (ESI): calc'd for $\mathrm{C}_{18} \mathrm{H}_{19} \mathrm{NO}_{2} \mathrm{SNa}\left([\mathrm{M}+\mathrm{Na}]^{+}\right)$336.1028. Found: 336.1043 .

41: IR (neat, $\mathrm{cm}^{-1}$ ): 3379, 2921, 1731, 1585, 1432, 1327, 1143. ${ }^{1} \mathrm{H}$ NMR (400 MHz, $\left.\mathrm{CDCl}_{3}\right) \delta 7.30-7.19(4 \mathrm{H}, \mathrm{m}), 7.03(1 \mathrm{H}, \mathrm{AB}, J=5.3 \mathrm{~Hz}), 7.01(1 \mathrm{H}, \mathrm{AB}, J=5.3 \mathrm{~Hz}), 6.39$ $(1 \mathrm{H}, \mathrm{s}), 2.20(3 \mathrm{H}, \mathrm{s}), 1.32(9 \mathrm{H}, \mathrm{s})$. 
5-o-Tolylthieno[2,3-b]pyrrole-6-carboxylic acid tert-butyl ester (38d)

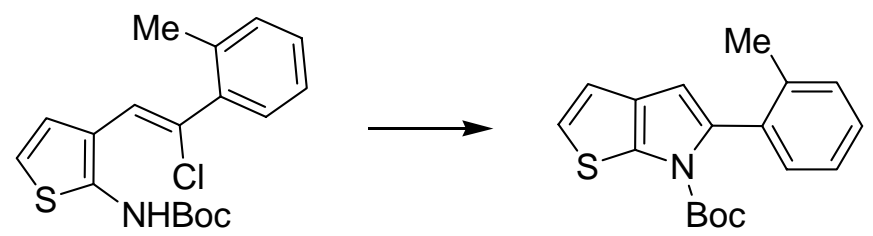

The general procedure for the tandem coupling was followed using 41 (10 mg, 0.0286 $\mathrm{mmol}), \mathrm{Cs}_{2} \mathrm{CO}_{3}$ (32.5 mg, $\left.0.10 \mathrm{mmol}\right), \mathrm{Pd}(\mathrm{OAc})_{2}(1.1 \mathrm{mg}, 0.005 \mathrm{mmol})$, and S-Phos (4.2 $\mathrm{mg}, 0.01 \mathrm{mmol})$. The reaction mixture was heated in PhMe $(1 \mathrm{~mL})$ at $100{ }^{\circ} \mathrm{C}$ for $14 \mathrm{~h}$. The crude material was separated using chromatography eluting with $3.5 \%$ EtOAc in hexanes to give $\mathbf{3 8 d}$ as an oil $(6.5 \mathrm{mg}, 73 \%)$.

\section{5-Phenyl-thieno[3,2-b]pyrrole-4-carboxylic acid tert-butyl ester (37a)}

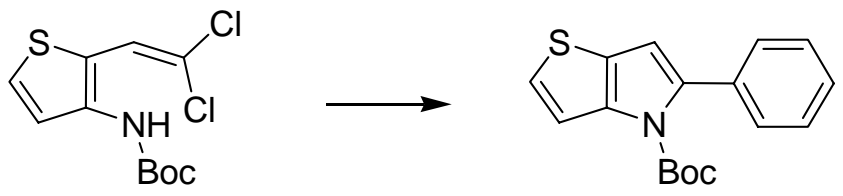

The general procedure for the tandem coupling was followed using 17 (73.6 mg, 0.25 mmol), phenylboronic acid (35 mg, $0.288 \mathrm{mmol}), \mathrm{K}_{3} \mathrm{PO}_{4} \cdot \mathrm{H}_{2} \mathrm{O}(288 \mathrm{mg}, 1.25 \mathrm{mmol})$, $\mathrm{Pd}(\mathrm{OAc})_{2}(1.68 \mathrm{mg}, 0.0075 \mathrm{mmol})$, and S-Phos $(6.2 \mathrm{mg}, 0.0150 \mathrm{mmol})$. The reaction mixture was heated in $\mathrm{PhMe}(2.5 \mathrm{~mL})$ at $100{ }^{\circ} \mathrm{C}$ for $20 \mathrm{~h}$. The crude material was purified using chromatography eluting with 3.5\% EtOAc in hexanes to give the product as a white solid (54.4 mg, 73\%). mp 103-104 ${ }^{\circ} \mathrm{C}$. IR (neat, $\mathrm{cm}^{-1}$ ): 2977, 1731, 1474, 1322, 1141. ${ }^{1} \mathrm{H}$ NMR (400 MHz, CDCl $) \delta$ 7.42-7.30 (6H, m), $7.18(1 \mathrm{H}, \mathrm{d}, J=5.1 \mathrm{~Hz}), 6.48(1 \mathrm{H}, \mathrm{d}, J=$ $0.7 \mathrm{~Hz}), 1.39$ (9H, s). ${ }^{13} \mathrm{C}$ NMR (100 MHz, $\left.\mathrm{CDCl}_{3}\right) \delta$ 149.4, 140.4, 139.6, 134.7, 129.4, 127.9, 127.7, 126.0, 124.6, 115.9, 107.9, 83.9, 27.9. HRMS (ESI): calc'd for $\mathrm{C}_{17} \mathrm{H}_{17} \mathrm{NO}_{2} \mathrm{NaS}\left([\mathrm{M}+\mathrm{Na}]^{+}\right)$322.0872. Found: 322.0876 .

5-(4-Fluorophenyl)-thieno[3,2-b]pyrrole-4-carboxylic acid tert-butyl ester (37b) 


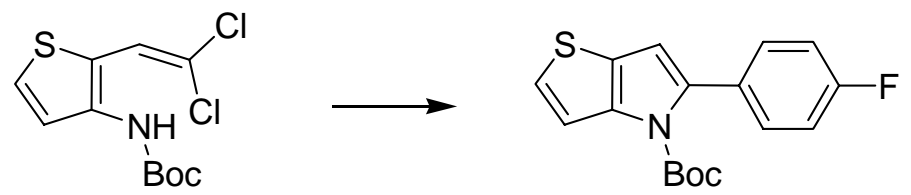

The general procedure for the tandem coupling was followed using 17 (70 mg, 0.238 mmol), 4-fluorophenylboronic acid (40 mg, $0.286 \mathrm{mmol}), \mathrm{K}_{3} \mathrm{PO}_{4} \cdot \mathrm{H}_{2} \mathrm{O}$ (274 mg, 1.2 $\mathrm{mmol}), \mathrm{Pd}(\mathrm{OAc})_{2}(2.66 \mathrm{mg}, 0.012 \mathrm{mmol})$, and S-Phos $(9.84 \mathrm{mg}, 0.024 \mathrm{mmol})$. The reaction mixture was heated in $\mathrm{PhMe}(2.5 \mathrm{~mL})$ at $100{ }^{\circ} \mathrm{C}$ for $4 \mathrm{~h}$. The crude material was purified using chromatography eluting with 3.5\% EtOAc in hexanes to give the product as a white solid (56 mg, 74\%). mp 122-123 ${ }^{\circ} \mathrm{C}$. IR (neat, $\mathrm{cm}^{-1}$ ): 2979, 1746, 1479, 1323, 1300, 1132. ${ }^{1} \mathrm{H}$ NMR (500 MHz, $\left.\mathrm{CDCl}_{3}\right) \delta 7.40(1 \mathrm{H}, \mathrm{d}, J=5.1 \mathrm{~Hz}), 7.38-7.36(2 \mathrm{H}, \mathrm{m})$, $7.19(1 \mathrm{H}, \mathrm{d}, J=5.3 \mathrm{~Hz}), 7.70\left(2 \mathrm{H}, \mathrm{tm}, J^{\mathrm{t}}=8.4 \mathrm{~Hz}\right), 6.46(1 \mathrm{H}, \mathrm{s}), 1.43(9 \mathrm{H}, \mathrm{s}) .{ }^{13} \mathrm{C} \mathrm{NMR}$ $\left(125 \mathrm{MHz}, \mathrm{CDCl}_{3}\right) \delta 162.5\left(J_{\mathrm{C}-\mathrm{F}}=247 \mathrm{~Hz}\right), 149.3,140.3,138.4,131.1\left(J_{\mathrm{C}-\mathrm{F}}=7.7 \mathrm{~Hz}\right)$, $130.7\left(J_{\mathrm{C}-\mathrm{F}}=3.4 \mathrm{~Hz}\right), 125.9,124.7,116.0,114.8\left(J_{\mathrm{C}-\mathrm{F}}=22 \mathrm{~Hz}\right), 108.0,84.1,28.1 .{ }^{19} \mathrm{~F}$ NMR (376 MHz, $\left.\mathrm{CDCl}_{3}\right) \delta$-114.4. HRMS (ESI): calc'd for $\mathrm{C}_{17} \mathrm{H}_{16} \mathrm{NO}_{2} \mathrm{FNaS}\left([\mathrm{M}+\mathrm{Na}]^{+}\right)$ 340.0778. Found: 340.0776 .

\section{5-(4-Trifluoromethylphenyl)-thieno[3,2-b]pyrrole-4-carboxylic acid tert-butyl ester} (37c)
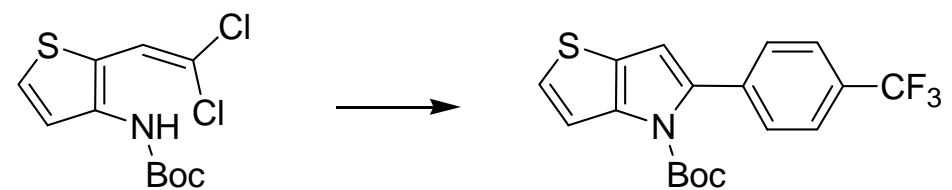

The general procedure for the tandem coupling was followed using 17 (73.6 mg, 0.25 $\mathrm{mmol}$ ), 4-trifluoromethylphenylboronic acid (54.6 mg, $0.29 \mathrm{mmol}), \mathrm{K}_{3} \mathrm{PO}_{4} \cdot \mathrm{H}_{2} \mathrm{O}(288 \mathrm{mg}$, $1.25 \mathrm{mmol}), \mathrm{Pd}(\mathrm{OAc})_{2}(1.68 \mathrm{mg}, 0.0075 \mathrm{mmol})$, and S-Phos $(6.2 \mathrm{mg}, 0.0150 \mathrm{mmol})$. The reaction mixture was heated in $\mathrm{PhMe}(2.5 \mathrm{~mL})$ at $100{ }^{\circ} \mathrm{C}$ for $7 \mathrm{~h}$. The crude material was purified using chromatography eluting with 4\% EtOAc in hexanes to give the product as a white solid (60.7 mg, 66\%). mp 119-120 ${ }^{\circ} \mathrm{C}$. IR (neat, $\mathrm{cm}^{-1}$ ): 2981, 1743, 1617, 1478, 1323, 1129. ${ }^{1} \mathrm{H}$ NMR (400 MHz, $\left.\mathrm{CDCl}_{3}\right) \delta 7.64(2 \mathrm{H}, \mathrm{dd}, J=8.6,0.7 \mathrm{~Hz}), 7.53(2 \mathrm{H}, \mathrm{dd}, J$ $=8.5,0.7 \mathrm{~Hz}), 7.41(1 \mathrm{H}, \mathrm{dd}, J=5.3,0.7 \mathrm{~Hz}), 7.23(1 \mathrm{H}, \mathrm{d}, J=5.3 \mathrm{~Hz}), 6.54(1 \mathrm{H}, \mathrm{s}), 1.43$ $(9 \mathrm{H}, \mathrm{s}) .{ }^{13} \mathrm{C}$ NMR $\left(125 \mathrm{MHz}, \mathrm{CDCl}_{3}\right) \delta 149.2,140.9,138.1,137.9,129.6\left(\mathrm{q}, J_{\mathrm{C}-\mathrm{F}}=37\right.$ 
$\mathrm{Hz}), 129.5,126.1,125.5,124.9\left(\mathrm{q}, J_{\mathrm{C}-\mathrm{F}}=3.8 \mathrm{~Hz}\right), 124.4\left(\mathrm{q}, J_{\mathrm{C}-\mathrm{F}}=272 \mathrm{~Hz}\right), 115.9,108.9$, 84.5, 28.0. ${ }^{19} \mathrm{~F}$ NMR (376 MHz, $\left.\mathrm{CDCl}_{3}\right) \delta-62.5$. HRMS (ESI): calc'd for $\mathrm{C}_{18} \mathrm{H}_{17} \mathrm{NO}_{2} \mathrm{~F}_{3} \mathrm{~S}$ $\left([\mathrm{M}+\mathrm{H}]^{+}\right)$368.0926. Found: 368.0931.

5-(4-Methoxycarbonylphenyl)thieno[3,2-b]pyrrole-4-carboxylic acid tert-butyl ester (37d) and 4-[2-(3-tert-butoxycarbonylaminothiophen-2-yl)-1-chlorovinyl]-benzoic acid methyl ester (42)



The general procedure for the tandem coupling was followed using 17 (73.6 mg, 0.25 mmol), 4-methoxycarbonylphenylboronic acid (51.8 mg, $0.29 \mathrm{mmol}), \mathrm{K}_{3} \mathrm{PO}_{4} \cdot \mathrm{H}_{2} \mathrm{O}(288$ $\mathrm{mg}, 1.25 \mathrm{mmol}), \mathrm{Pd}(\mathrm{OAc})_{2}(1.68 \mathrm{mg}, 0.0075 \mathrm{mmol})$, and S-Phos (6.2 mg, $\left.0.0150 \mathrm{mmol}\right)$. The reaction mixture was heated in $\mathrm{PhMe}(2.5 \mathrm{~mL})$ at $100{ }^{\circ} \mathrm{C}$ for $14 \mathrm{~h}$. The crude material was separated using chromatography eluting with $5 \% \rightarrow 10 \%$ EtOAc in hexanes to give 37d as a white solid (less polar, $11 \mathrm{mg}, 12 \%$ ) and 42 as an oil (more polar, 56.4 $\mathrm{mg}, 57 \%)$.

37d: $\mathrm{mp} 86-87{ }^{\circ} \mathrm{C}$. IR (neat, $\mathrm{cm}^{-1}$ ): 2980, 1726, 1609, 1321, 1276, 1141. ${ }^{1} \mathrm{H}$ NMR $\left(500 \mathrm{MHz}, \mathrm{CDCl}_{3}\right) \delta 8.05\left(2 \mathrm{H}, \mathrm{dm}, J^{\mathrm{d}}=8.7 \mathrm{~Hz}\right), 7.48\left(2 \mathrm{H}, \mathrm{dm}, J^{\mathrm{d}}=8.6 \mathrm{~Hz}\right), 7.41(1 \mathrm{H}, \mathrm{d}$, $J=5.2 \mathrm{~Hz}), 7.23(1 \mathrm{H}, \mathrm{d}, J=5.2 \mathrm{~Hz}), 6.56(1 \mathrm{H}, \mathrm{s}), 3.94(3 \mathrm{H}, \mathrm{s}), 1.42(9 \mathrm{H}, \mathrm{s}) .{ }^{13} \mathrm{C} \mathrm{NMR}$ $\left(125 \mathrm{MHz}, \mathrm{CDCl}_{3}\right) \delta 167.1,149.2,141.0,139.0,138.4,129.2,129.0,126.1,125.4,115.8$, 108.9, 84.4, 52.4, 28.0. HRMS (ESI): calc'd for $\mathrm{C}_{19} \mathrm{H}_{19} \mathrm{NO}_{4} \mathrm{NaS}\left([\mathrm{M}+\mathrm{Na}]^{+}\right) 380.0927$. Found: 380.0925 .

42: ${ }^{1} \mathrm{H}$ NMR $\left(500 \mathrm{MHz}, \mathrm{CDCl}_{3}\right) \delta 8.04\left(2 \mathrm{H}, \mathrm{dm}, J^{\mathrm{d}}=8.4 \mathrm{~Hz}\right), 7.75\left(2 \mathrm{H}, \mathrm{dm}, J^{\mathrm{d}}=8.4\right.$ Hz), $7.38(1 \mathrm{H}, \mathrm{d}, J=4.6 \mathrm{~Hz}), 7.37(1 \mathrm{H}, \mathrm{br}), 7.25(1 \mathrm{H}, \mathrm{s}), 6.55(1 \mathrm{H}, \mathrm{br}), 3.93(3 \mathrm{H}, \mathrm{s}), 1.52$ $\left(9 \mathrm{H}\right.$, s). HRMS (ESI): calc'd for $\mathrm{C}_{19} \mathrm{H}_{20} \mathrm{NO}_{4} \mathrm{NaSCl}\left([\mathrm{M}+\mathrm{Na}]^{+}\right)$416.0693. Found: 416.0683. 
5-(4-Methoxycarbonylphenyl)thieno[3,2-b]pyrrole-4-carboxylic acid tert-butyl ester (37d)

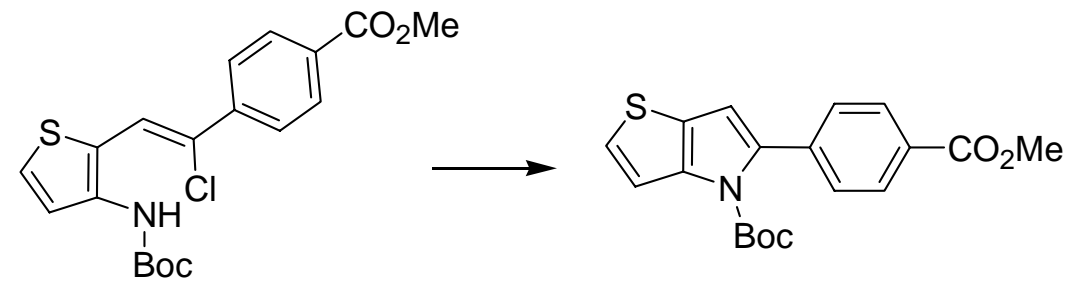

The general procedure for the tandem coupling was followed using $42(56.4 \mathrm{mg}$, $0.143 \mathrm{mmol}), \mathrm{K}_{3} \mathrm{PO}_{4} \cdot \mathrm{H}_{2} \mathrm{O}$ (66 mg, $\left.0.286 \mathrm{mmol}\right), \mathrm{Pd}(\mathrm{OAc})_{2}(1.6 \mathrm{mg}, 0.00715 \mathrm{mmol})$, and S-Phos $(5.9 \mathrm{mg}, 0.0143 \mathrm{mmol})$. The reaction mixture was heated in PhMe $(2.5 \mathrm{~mL})$ at $100{ }^{\circ} \mathrm{C}$ for $7 \mathrm{~h}$. The crude material was purified using chromatography eluting with $5 \% \rightarrow 10 \%$ EtOAc in hexanes to give $\mathbf{3 7 d}$ as a white solid (38 $\mathrm{mg}, 74 \%$ ).

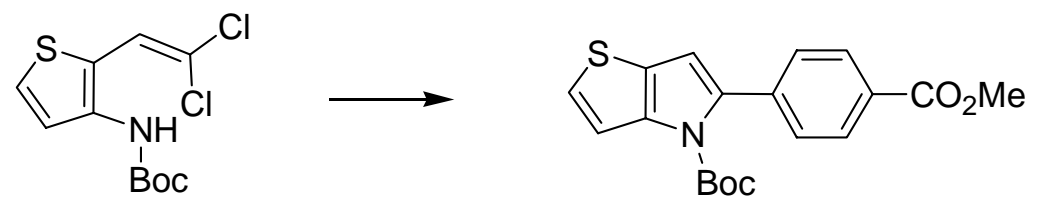

The general procedure for the tandem coupling was followed using 17 (73.6 mg, 0.25 mmol), 4-methoxycarbonylphenylboronic acid $(51.8 \mathrm{mg}, 0.29 \mathrm{mmol}), \mathrm{K}_{3} \mathrm{PO}_{4} \cdot \mathrm{H}_{2} \mathrm{O}(288$ $\mathrm{mg}, 1.25 \mathrm{mmol}), \mathrm{Pd}(\mathrm{OAc})_{2}(1.2 \mathrm{mg}, 0.005 \mathrm{mmol})$, and S-Phos $(4.1 \mathrm{mg}, 0.010 \mathrm{mmol})$. The reaction mixture was heated in $\mathrm{PhMe}(2.5 \mathrm{~mL})$ at $100{ }^{\circ} \mathrm{C}$ for $4.5 \mathrm{~h}$ and an additional portion of catalyst solution $\left[\mathrm{Pd}(\mathrm{OAc})_{2}(1.68 \mathrm{mg}, 0.0075 \mathrm{mmol})\right.$, and S-Phos $(6.2 \mathrm{mg}$, $0.015 \mathrm{mmol})$ in $\mathrm{PhMe}(1 \mathrm{~mL})]$ was added. The reaction was heated at $100{ }^{\circ} \mathrm{C}$ for an addition $3 \mathrm{~h}$. The crude material was purified using chromatography eluting with $10 \%$ EtOAc in hexanes to give $\mathbf{3 7 d}$ as a white solid (66.4 mg, 74\%).

\section{5-Pent-1-enylthieno[3,2-b]pyrrole-4-carboxylic acid tert-butyl ester (37e)}

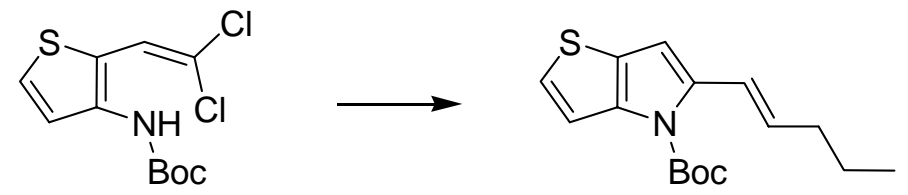

The general procedure for the tandem coupling was followed using 17 (73.6 mg, 0.25 mmol), 1-pentenylboronic acid (34.2 mg, $0.30 \mathrm{mmol}), \mathrm{K}_{3} \mathrm{PO}_{4} \cdot \mathrm{H}_{2} \mathrm{O}(288 \mathrm{mg}, 1.25 \mathrm{mmol})$, 
$\mathrm{Pd}(\mathrm{OAc})_{2}(2.8 \mathrm{mg}, 0.0125 \mathrm{mmol})$, and $\mathrm{S}-\mathrm{Phos}(10.3 \mathrm{mg}, 0.025 \mathrm{mmol})$. The reaction mixture was heated in $\mathrm{PhMe}(2.5 \mathrm{~mL})$ at $100{ }^{\circ} \mathrm{C}$ for $16 \mathrm{~h}$. The crude material was purified using chromatography eluting with $2.5 \%$ EtOAc in hexanes to give 37 e as a colorless oil (58 mg, 80\%). IR (neat, $\mathrm{cm}^{-1}$ ): 2963, 1738, 1485, 1370, 1320, 1255, 1120. ${ }^{1} \mathrm{H}$ NMR (400 $\left.\mathrm{MHz} \mathrm{CDCl}_{3}\right) \delta 7.27(1 \mathrm{H}, \mathrm{d}, J=5.1 \mathrm{~Hz}), 7.08(1 \mathrm{H}, \mathrm{d}, J=5.1 \mathrm{~Hz}), 6.99(1 \mathrm{H}, \mathrm{dd}, J=15.8$, $0.7 \mathrm{~Hz}), 6.59(1 \mathrm{H}, \mathrm{s}), 6.09\left(1 \mathrm{H}, \mathrm{dt}, J^{\mathrm{d}}=15.8 \mathrm{~Hz}, J^{\mathrm{t}}=6.9 \mathrm{~Hz}\right), 2.20\left(2 \mathrm{H}, \mathrm{qd}, J^{\mathrm{q}}=7.6 \mathrm{~Hz}, J^{\mathrm{d}}\right.$ $=0.4 \mathrm{~Hz}), 1.66(9 \mathrm{H}, \mathrm{s}), 1.50(2 \mathrm{H}$, sextet, $J=7.4 \mathrm{~Hz}), 0.96(3 \mathrm{H}, \mathrm{t}, J=7.4 \mathrm{~Hz}) .{ }^{13} \mathrm{C} \mathrm{NMR}$ $\left(125 \mathrm{MHz}, \mathrm{CDCl}_{3}\right) \delta 149.6,139.4,139.1,132.3,126.4,123.6,122.0,116.2,103.4,84.1$, 35.3, 28.4, 22.7, 14.0. HRMS (EI): calc'd for $\mathrm{C}_{16} \mathrm{H}_{21} \mathrm{NO}_{2} \mathrm{~S}$ ([M] $\left.]^{+}\right)$291.1293. Found: 291.1290.

\section{5-Thiophen-3-yl-thieno[3,2-b]pyrrole-4-carboxylic acid tert-butyl ester (37f)}

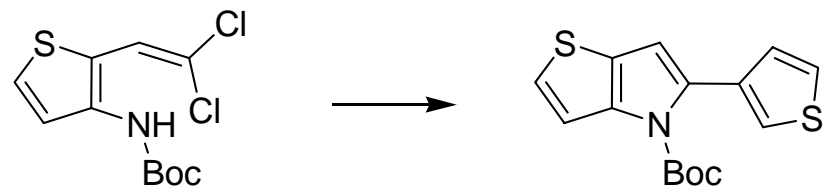

The general procedure for the tandem coupling was followed using 17 (70 mg, 0.238 mmol), 3-thienylboronic acid (36.6 mg, $0.286 \mathrm{mmol}), \mathrm{K}_{3} \mathrm{PO}_{4} \cdot \mathrm{H}_{2} \mathrm{O}(274 \mathrm{mg}, 1.2 \mathrm{mmol})$, $\mathrm{Pd}(\mathrm{OAc})_{2}(2.66 \mathrm{mg}, 0.012 \mathrm{mmol})$, and $\mathrm{S}-\mathrm{Phos}(9.8 \mathrm{mg}, 0.024 \mathrm{mmol})$. The reaction mixture was heated in $\mathrm{PhMe}(2.5 \mathrm{~mL})$ at $100{ }^{\circ} \mathrm{C}$ for $6 \mathrm{~h}$. The crude material was purified using chromatography eluting with $2.5 \%$ EtOAc in hexanes to give $37 \mathrm{f}$ as a white solid (53 mg, 73\%). mp 111-112 ${ }^{\circ} \mathrm{C}$. IR (neat, $\mathrm{cm}^{-1}$ ): 3099, 2978, 1731, 1486, 1319, 1131. ${ }^{1} \mathrm{H}$ NMR (400 MHz, $\left.\mathrm{CDCl}_{3}\right) \delta 7.40(1 \mathrm{H}, \mathrm{d}, J=5.1 \mathrm{~Hz}), 7.32-7.30(2 \mathrm{H}, \mathrm{m}), 7.19(1 \mathrm{H}, \mathrm{d}, J=$ $5.1 \mathrm{~Hz}), 7.16(1 \mathrm{H}, \mathrm{dd}, J=4.6,1.1 \mathrm{~Hz}), 6.53(1 \mathrm{H}, \mathrm{s}), 1.49(9 \mathrm{H}, \mathrm{s}) .{ }^{13} \mathrm{C}$ NMR $(100 \mathrm{MHz}$, $\left.\mathrm{CDCl}_{3}\right) \delta 149.3,140.1,134.7,134.3,129.6,125.9,124.6,124.4,123.5,116.0,108.1$, 84.0, 28.0. HRMS (EI): calc'd for $\mathrm{C}_{15} \mathrm{H}_{25} \mathrm{NO}_{2} \mathrm{~S}_{2}\left([\mathrm{M}]^{+}\right)$305.0544. Found: 305.0547.

\section{5-Phenyl-6H-thieno[2,3-b]pyrrole (39)}
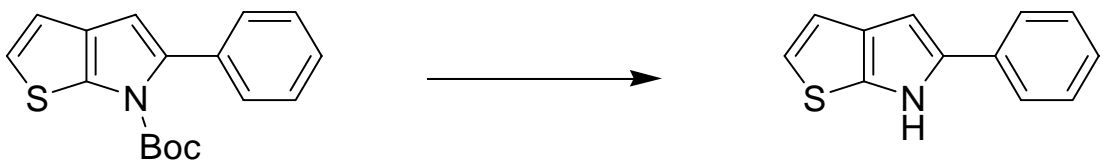
To a 5-mL round-bottomed flask was charged 38a (36.2 $\mathrm{mg}, 0.12 \mathrm{mmol})$ and $\mathrm{MeONa}$ (65 mg, $1.2 \mathrm{mmol})$. After purged with argon for $5 \mathrm{~min}$, anhydrous methanol $(1 \mathrm{~mL})$ and THF $(1 \mathrm{~mL})$ were added. The mixture was stirred at $\mathrm{rt}$ overnight $(16 \mathrm{~h})$, quenched with $\mathrm{NaHCO}_{3}$, extracted with $\mathrm{Et}_{2} \mathrm{O}(3 \times 10 \mathrm{~mL})$, dried over $\mathrm{MgSO}_{4}$. The crude material was chromatographed with 10\% EtOAc/hexanes to afford a white solid $(23.1 \mathrm{mg}, 96 \%) .{ }^{1} \mathrm{H}$ NMR (400 MHz, $\left.\mathrm{CDCl}_{3}\right) \delta 8.43(1 \mathrm{H}, \mathrm{br}), 7.50(2 \mathrm{H}, \mathrm{dm}, J=8.0 \mathrm{~Hz}), 7.37(2 \mathrm{H}, \mathrm{t}, J=7.9$ $\mathrm{Hz}), 7.50\left(1 \mathrm{H}, \mathrm{tm}, J^{\mathrm{t}}=7.4 \mathrm{~Hz}\right), 6.99(1 \mathrm{H}, \mathrm{d}, J=5.3 \mathrm{~Hz}), 6.83(1 \mathrm{H}, \mathrm{dd}, J=5.3,0.5 \mathrm{~Hz})$, $6.71(1 \mathrm{H}, \mathrm{d}, J=2.0 \mathrm{~Hz}) .{ }^{13} \mathrm{C} \mathrm{NMR}\left(100 \mathrm{MHz}, \mathrm{CDCl}_{3}\right) \delta 138.8,134.9,133.2,132.6$, $129.2,126.9,124.3,118.6,118.0,99.2$. 


\section{Crystallographic information for 38b}

Identification code

Empirical formula

Formula weight

Temperature

Wavelength

Crystal system

Space group

Unit cell dimensions

Volume

Z

Density (calculated)

Absorption coefficient

$\mathrm{F}(000)$

Crystal size

Theta range for data collection

Index ranges

Reflections collected

Independent reflections

Completeness to theta $=27.48^{\circ}$

Absorption correction

Max. and min. transmission

Refinement method

Data / restraints / parameters

Goodness-of-fit on $\mathrm{F}^{2}$

Final R indices [I $>2 \operatorname{sigma}(\mathrm{I})]$

$\mathrm{R}$ indices (all data)

Largest diff. peak and hole k0693

C17 H16 F N O2 S

317.37

150(1) K

$0.71073 \AA$

Monoclinic

P 21/n

$\mathrm{a}=11.0547(7) \AA$

$\alpha=90^{\circ}$.

$\mathrm{b}=8.6515(4) \AA$ $\beta=106.756(3)^{\circ}$.

$\mathrm{c}=17.2969(13) \AA$ $\gamma=90^{\circ}$.

1584.03(17) $\AA^{3}$

4

$1.331 \mathrm{Mg} / \mathrm{m}^{3}$

$0.221 \mathrm{~mm}^{-1}$

664

$0.48 \times 0.30 \times 0.18 \mathrm{~mm}^{3}$

2.56 to $27.48^{\circ}$.

$-14<=\mathrm{h}<=13,-11<=\mathrm{k}<=11,-18<=1<=22$

11193

$3616[\mathrm{R}(\mathrm{int})=0.0703]$

$99.5 \%$

Semi-empirical from equivalents

0.970 and 0.702

Full-matrix least-squares on $\mathrm{F}^{2}$

$3616 / 0 / 202$

1.029

$\mathrm{R} 1=0.0546, \mathrm{wR} 2=0.1276$

$\mathrm{R} 1=0.1185, \mathrm{wR} 2=0.1552$

0.222 and -0.458 e. $\AA^{-3}$ 
- S36 -

Two different ORTEP views of $38 b$
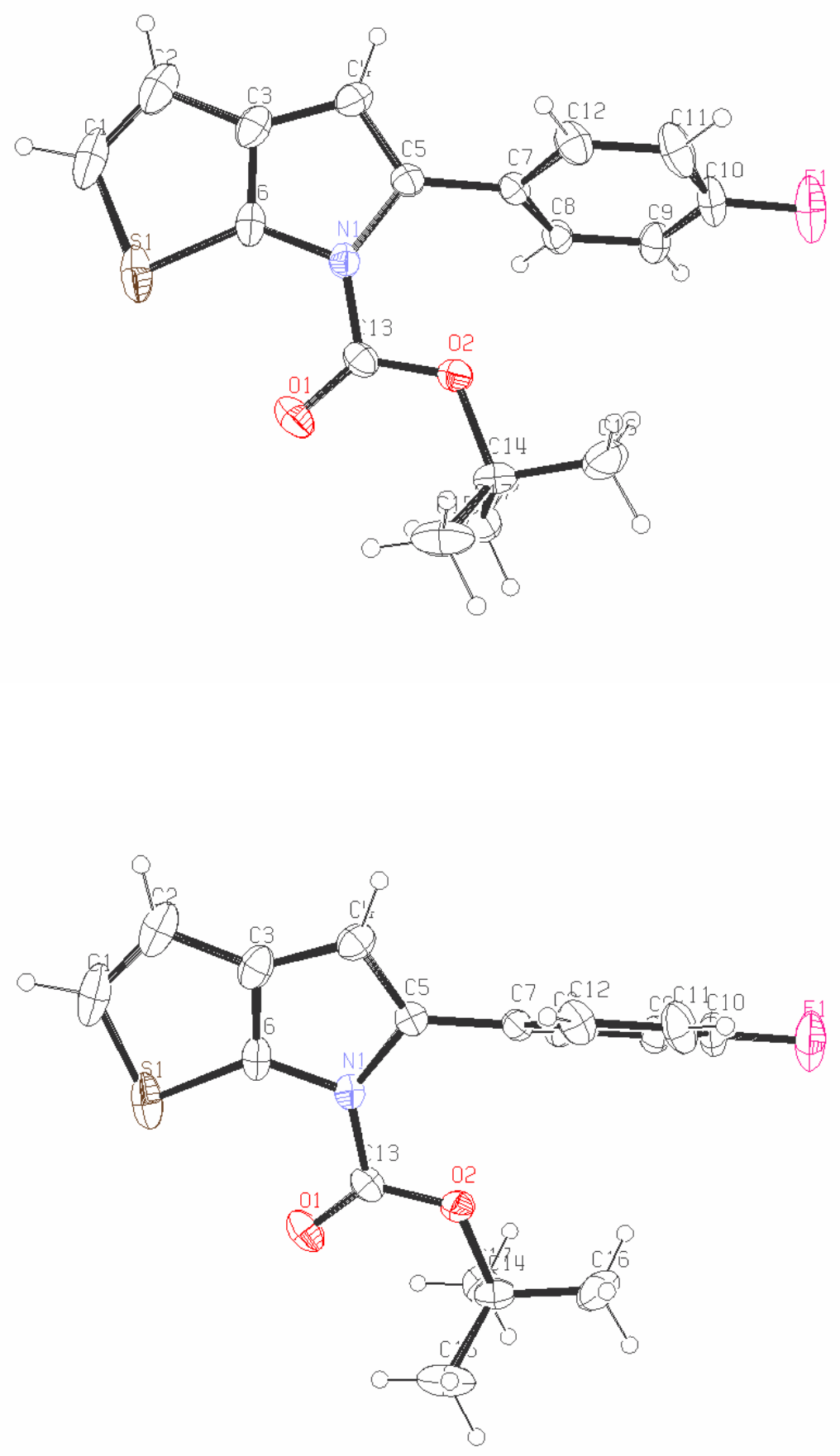

36 

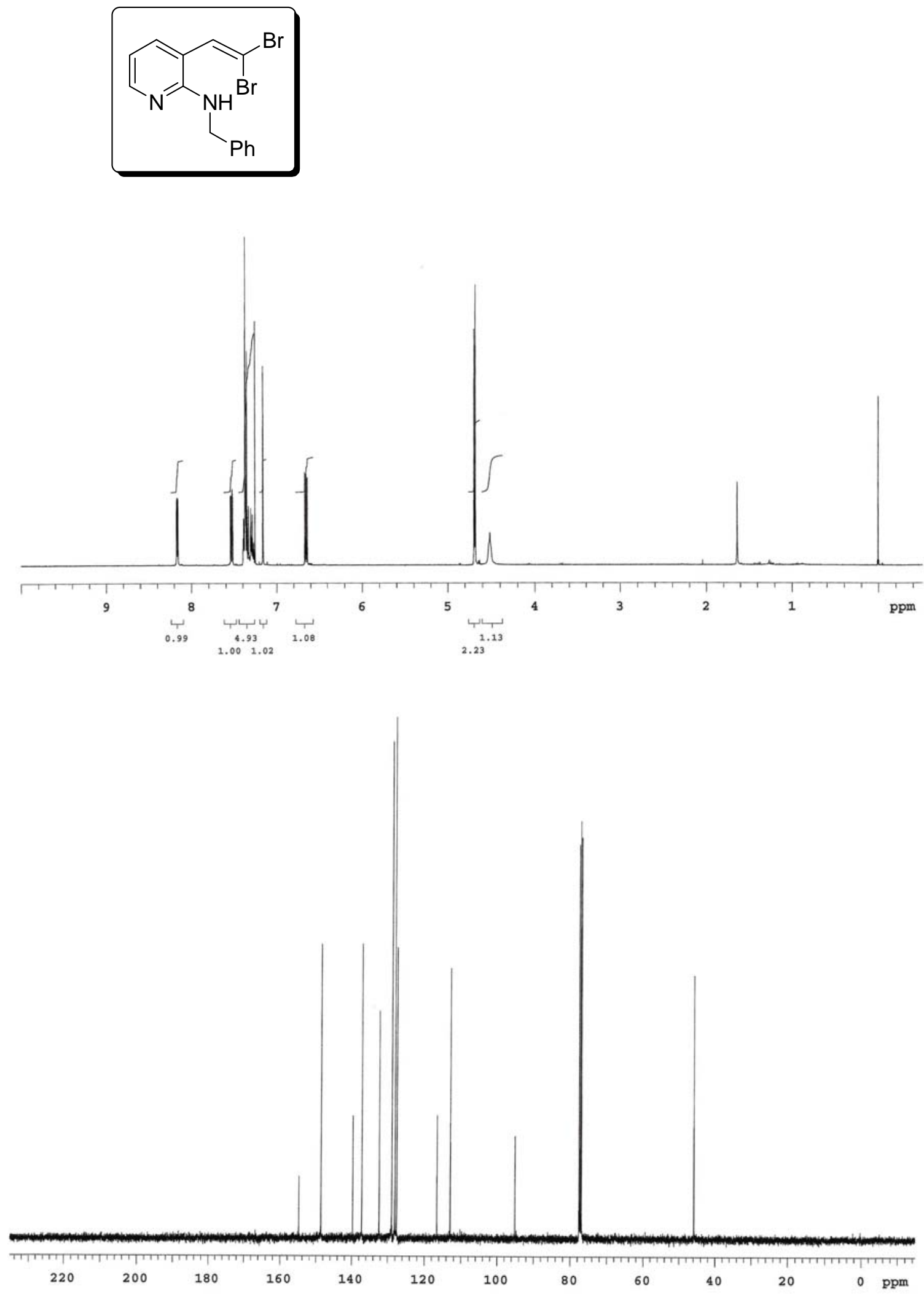
- S38 -
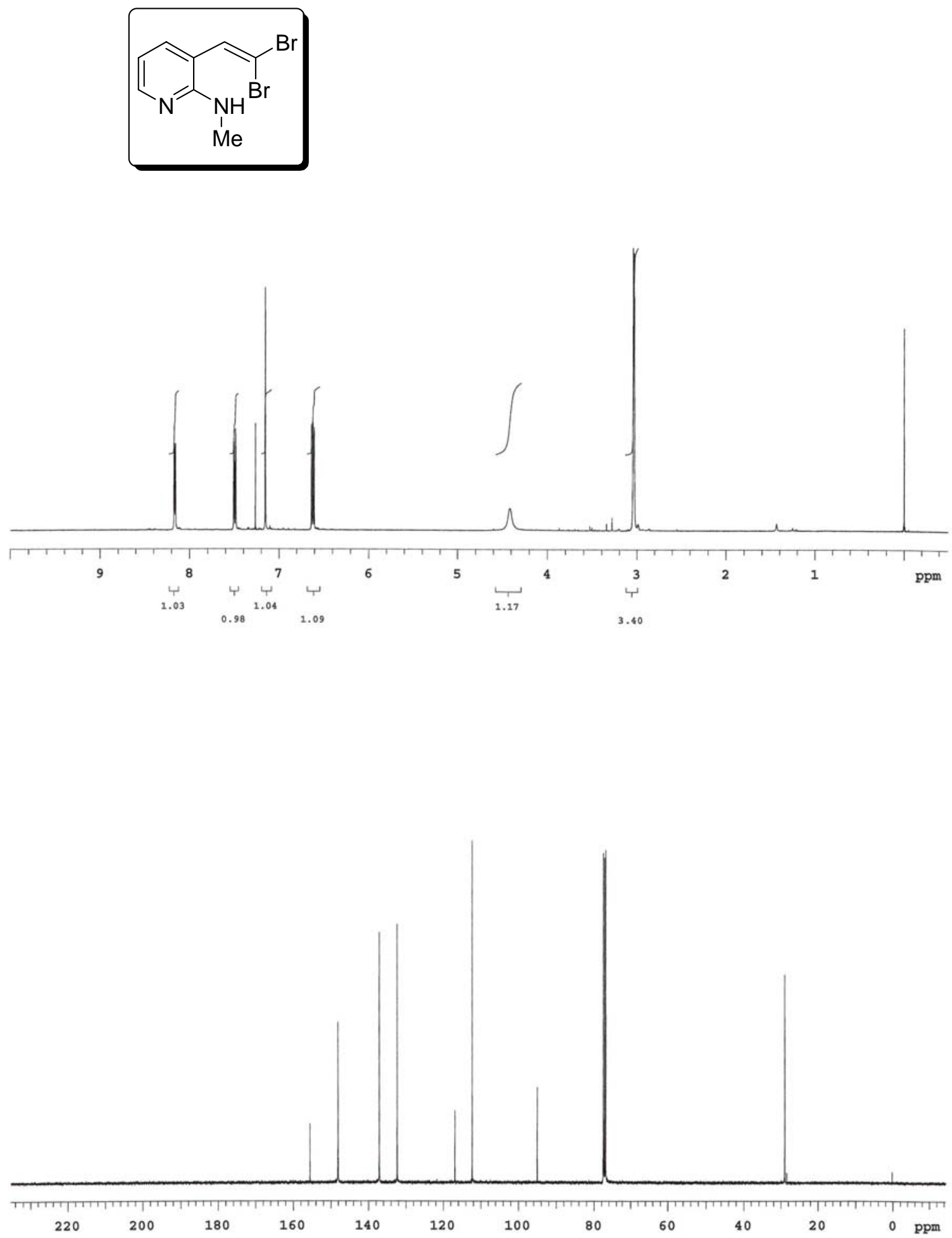
- S39 -
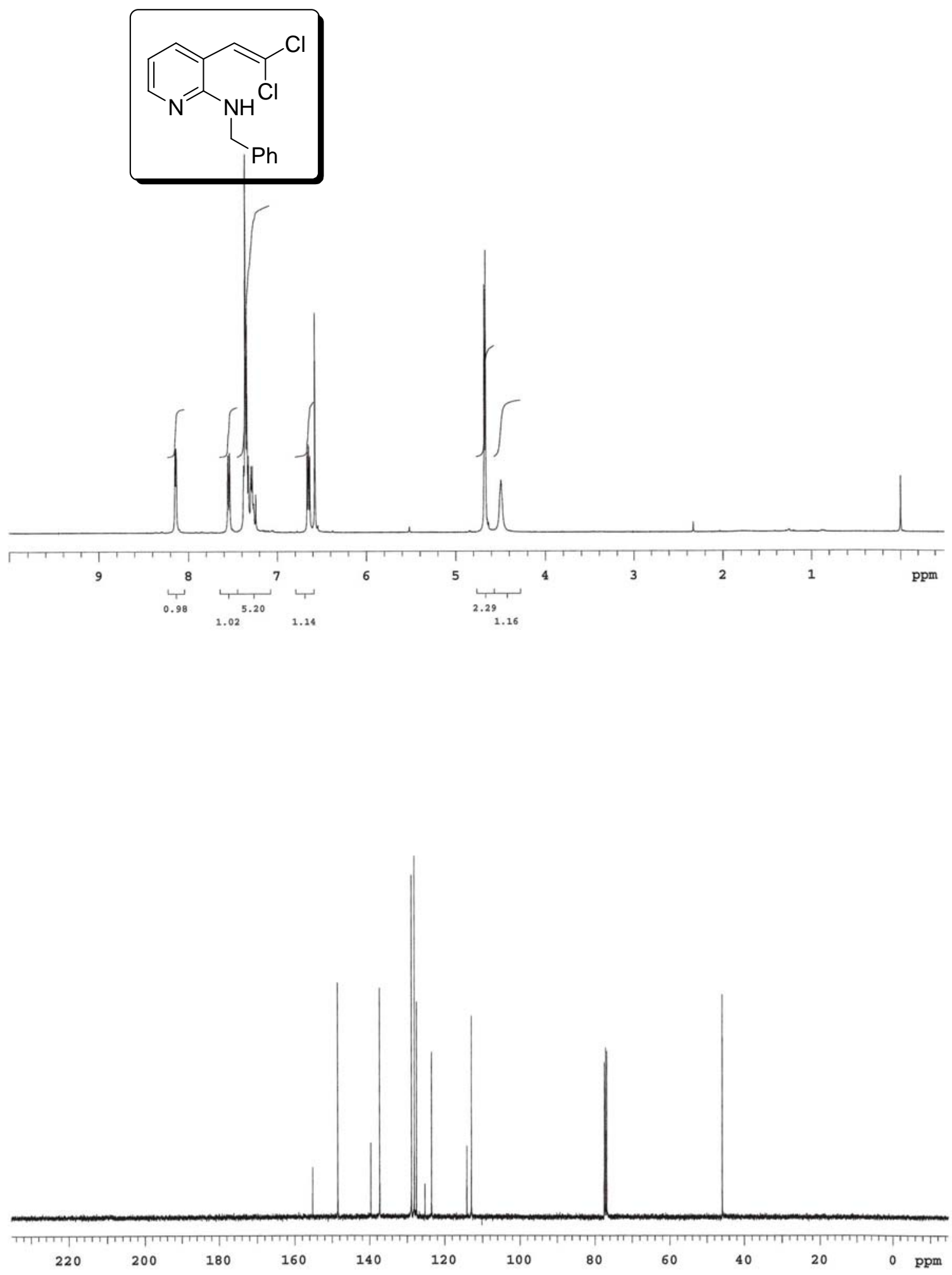


- S41 -
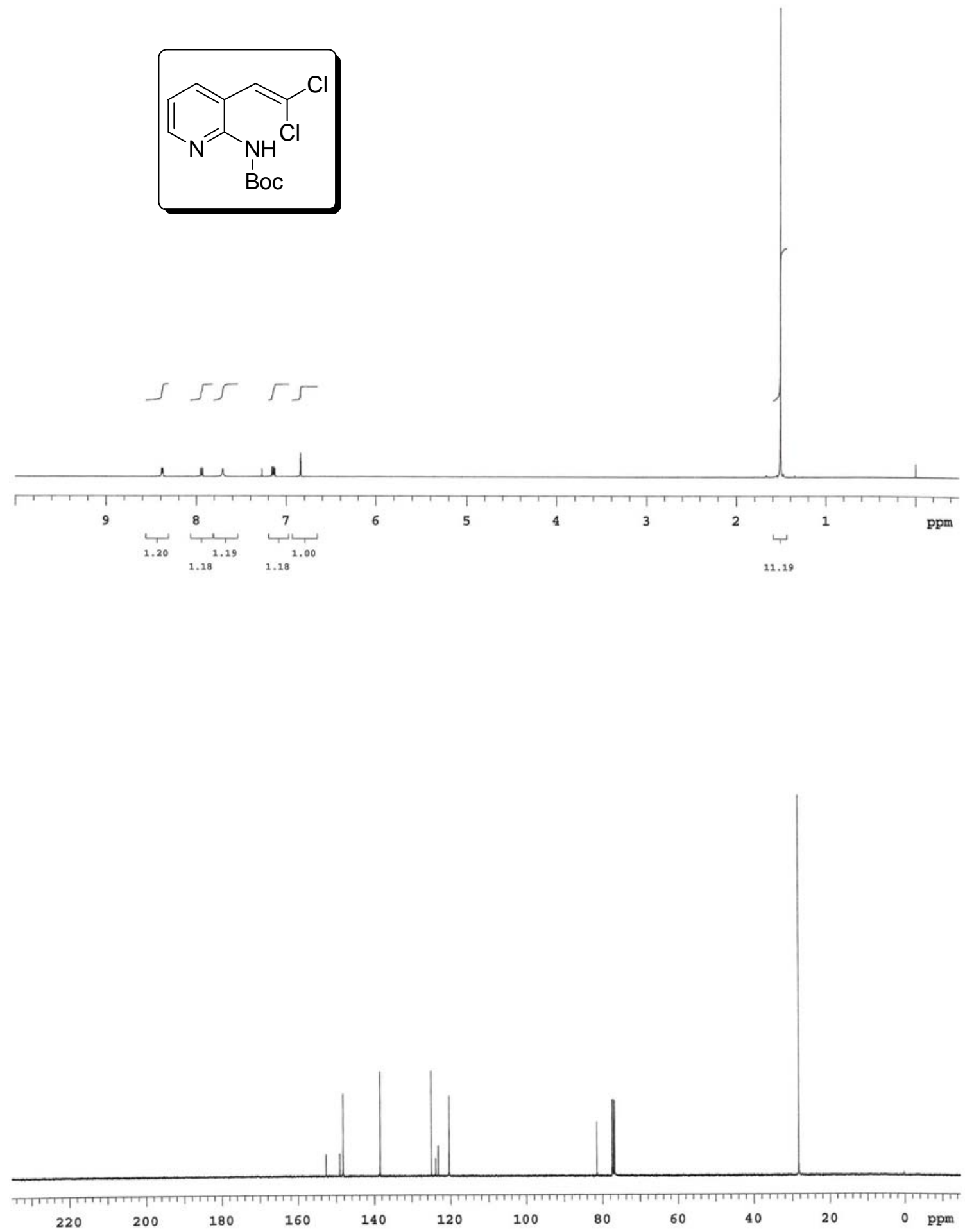
- S42 -
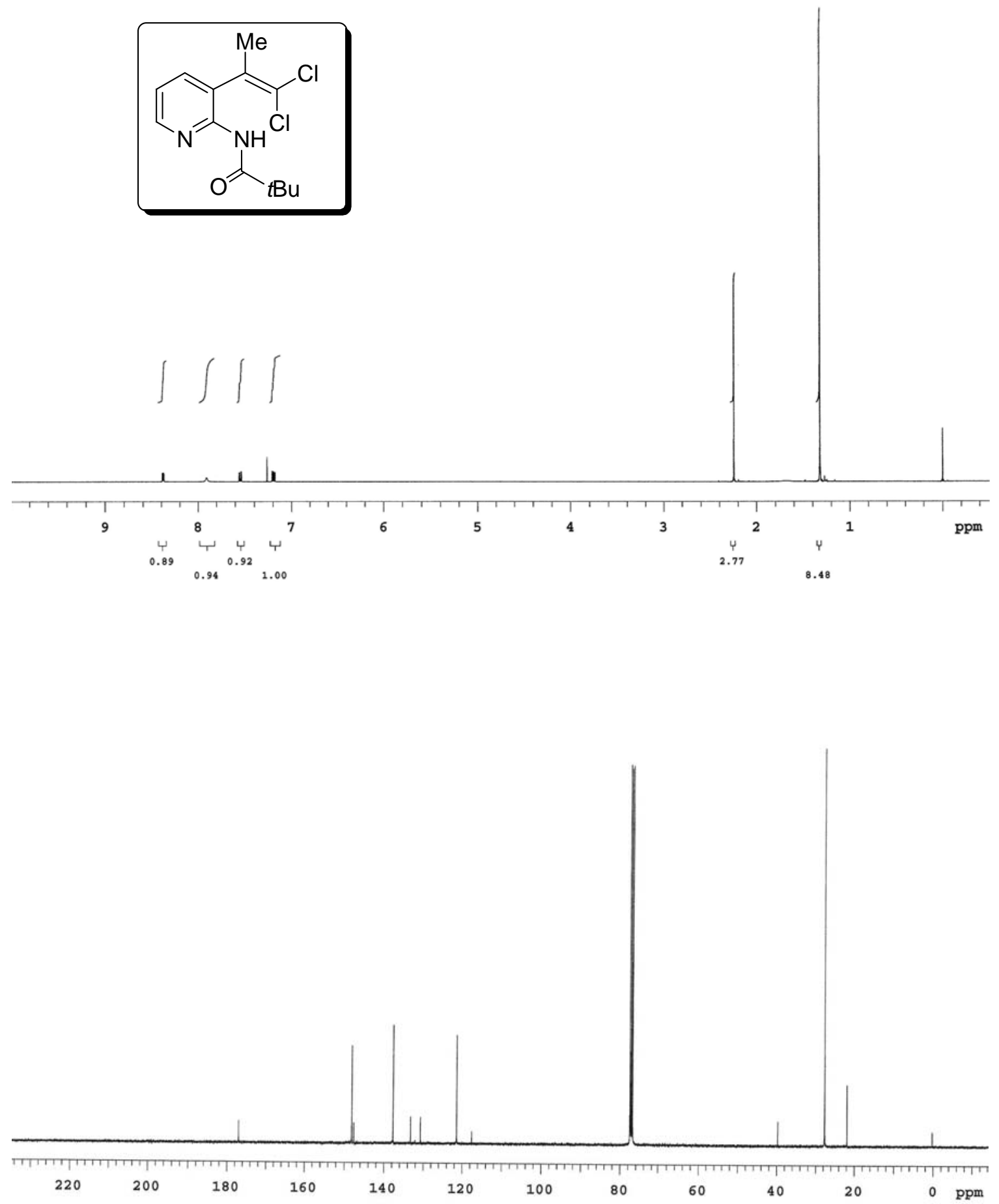
- S43 -
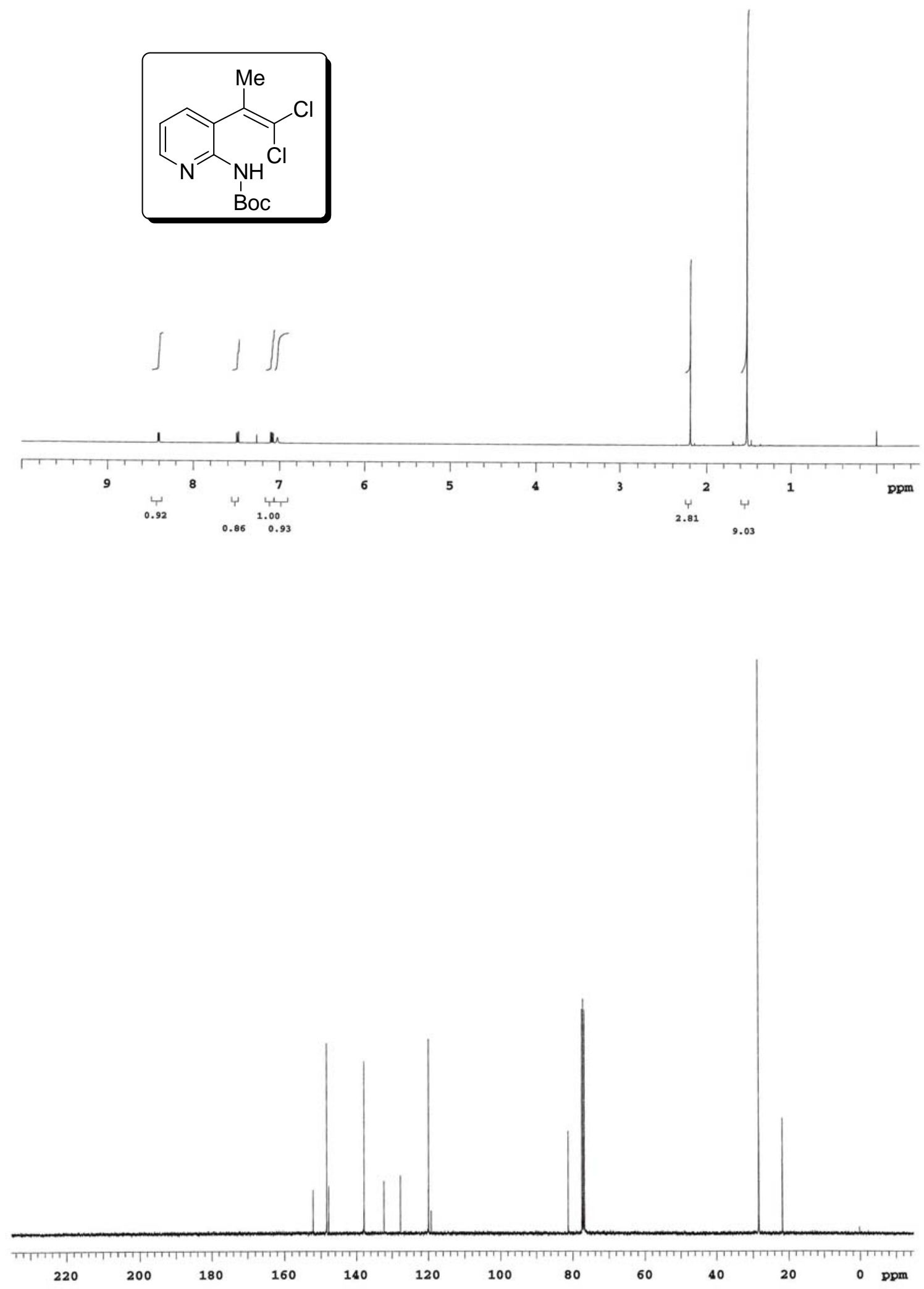
- S44 -
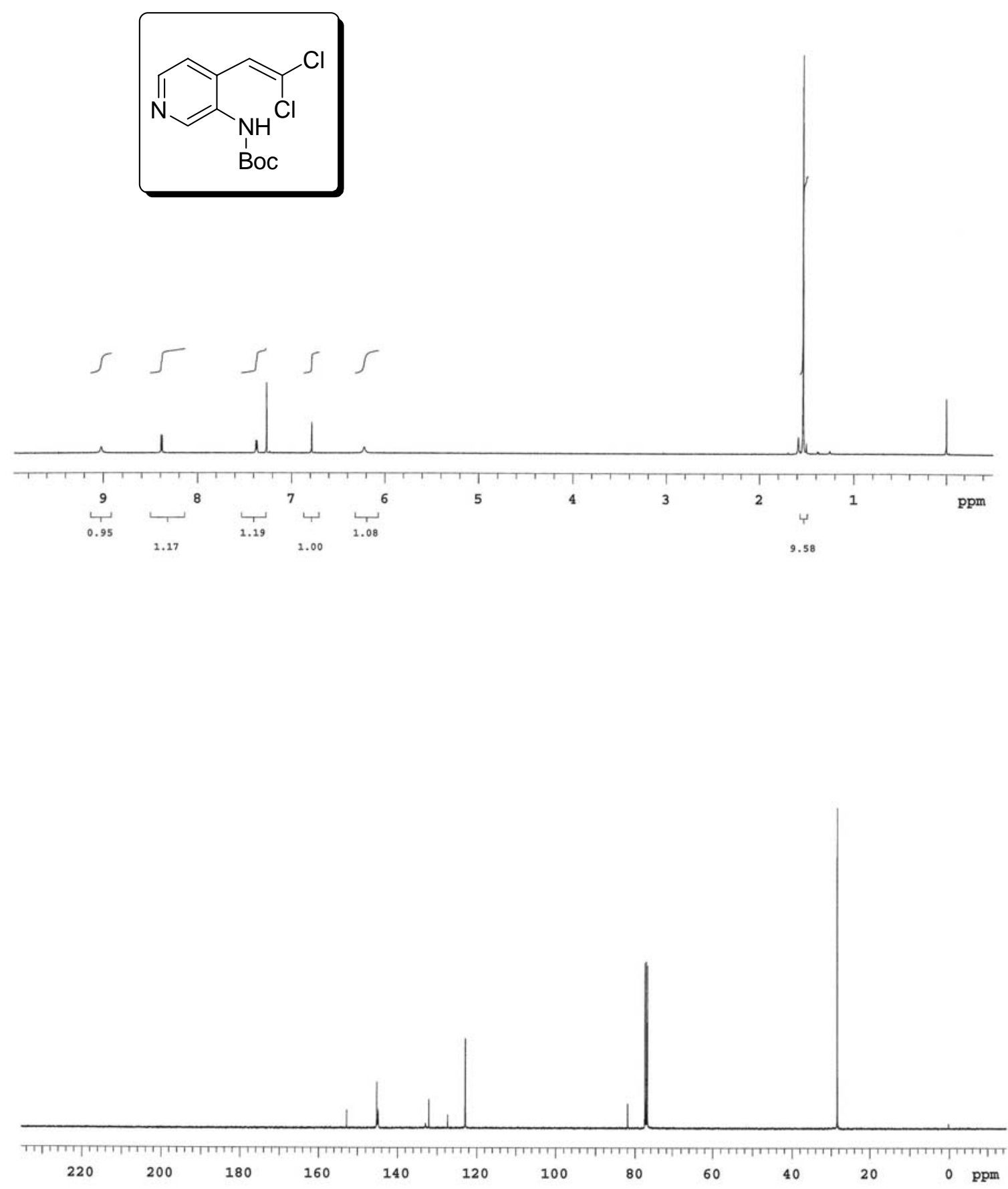
- S45 -
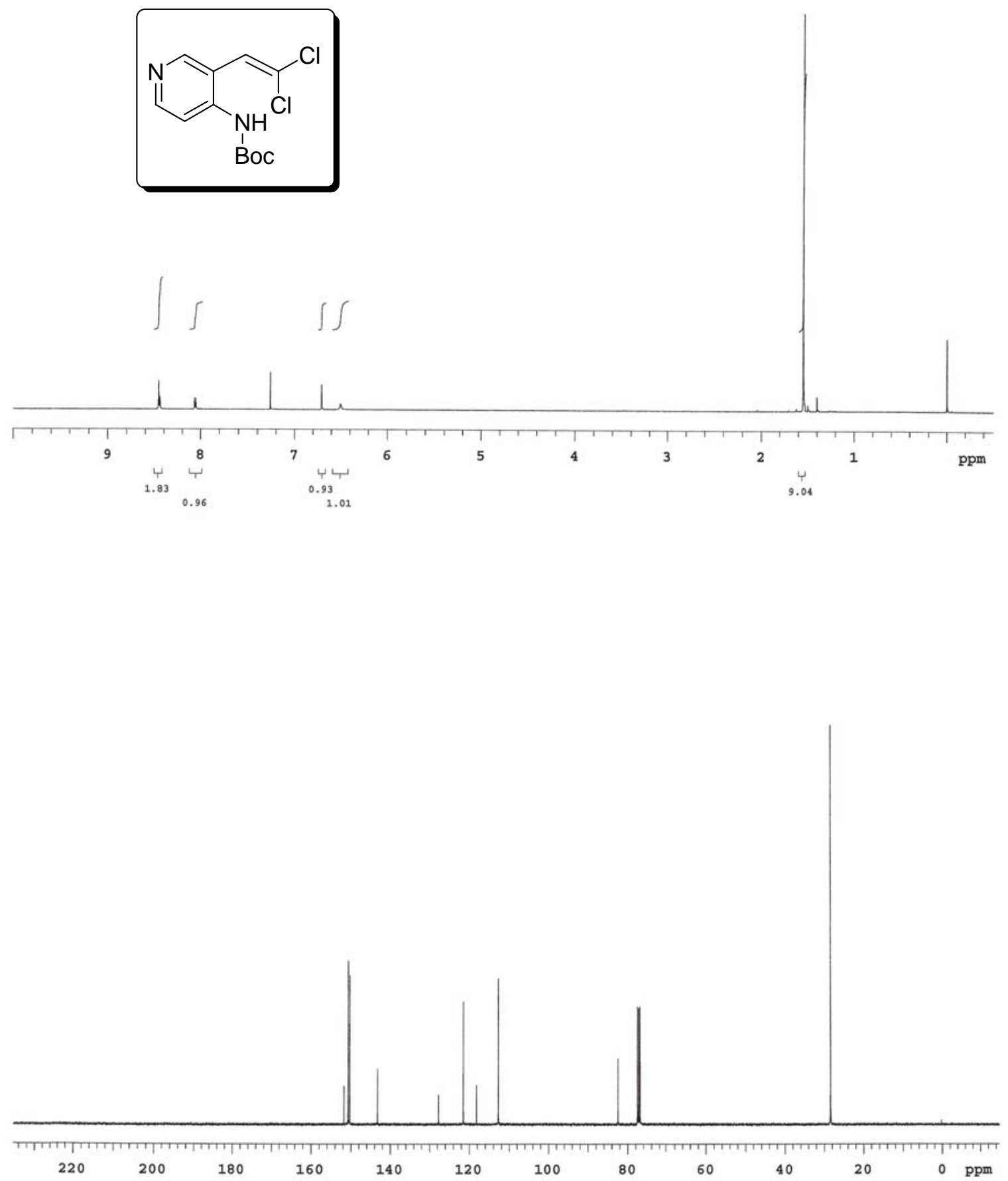
- S46 -
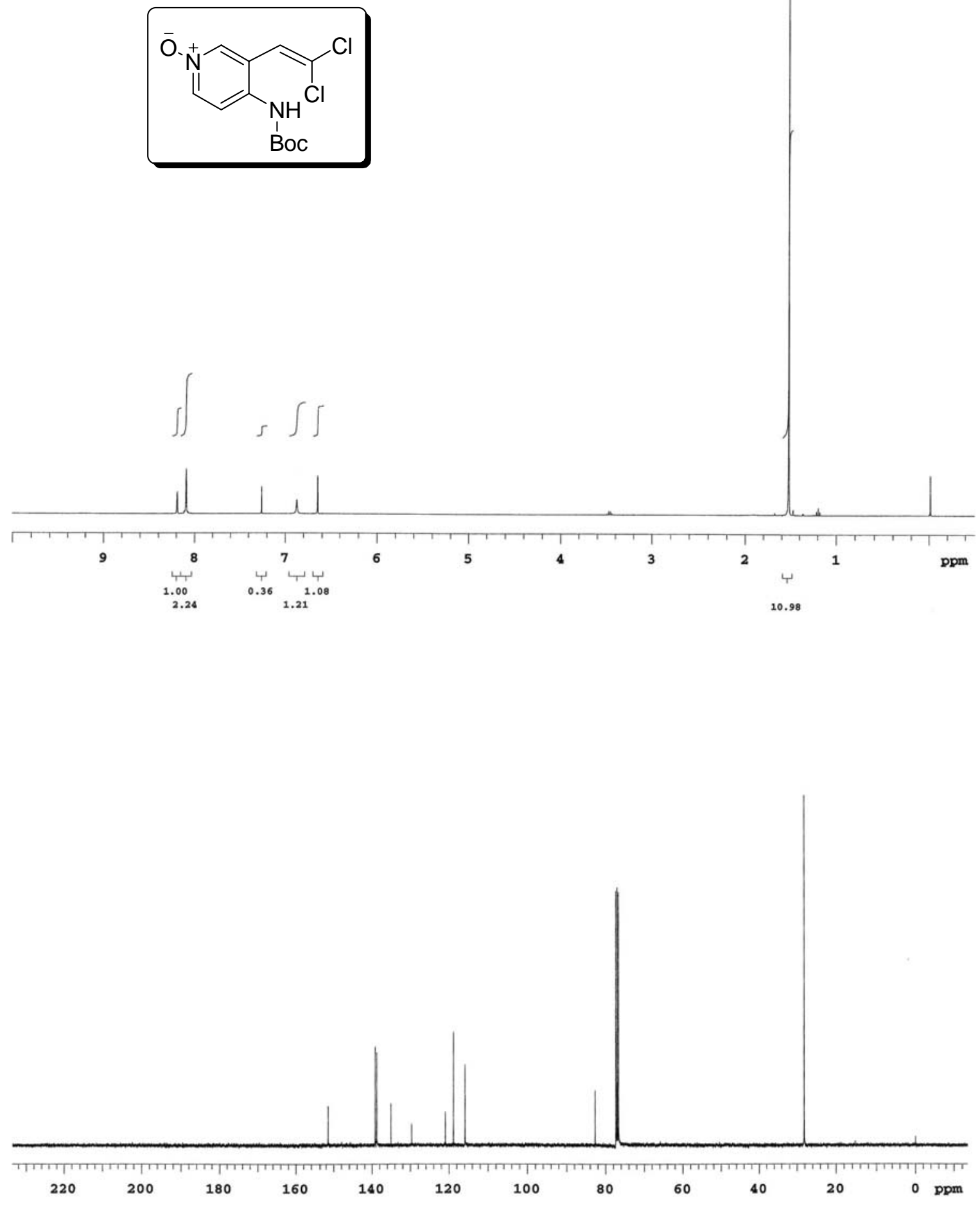
- S47 -
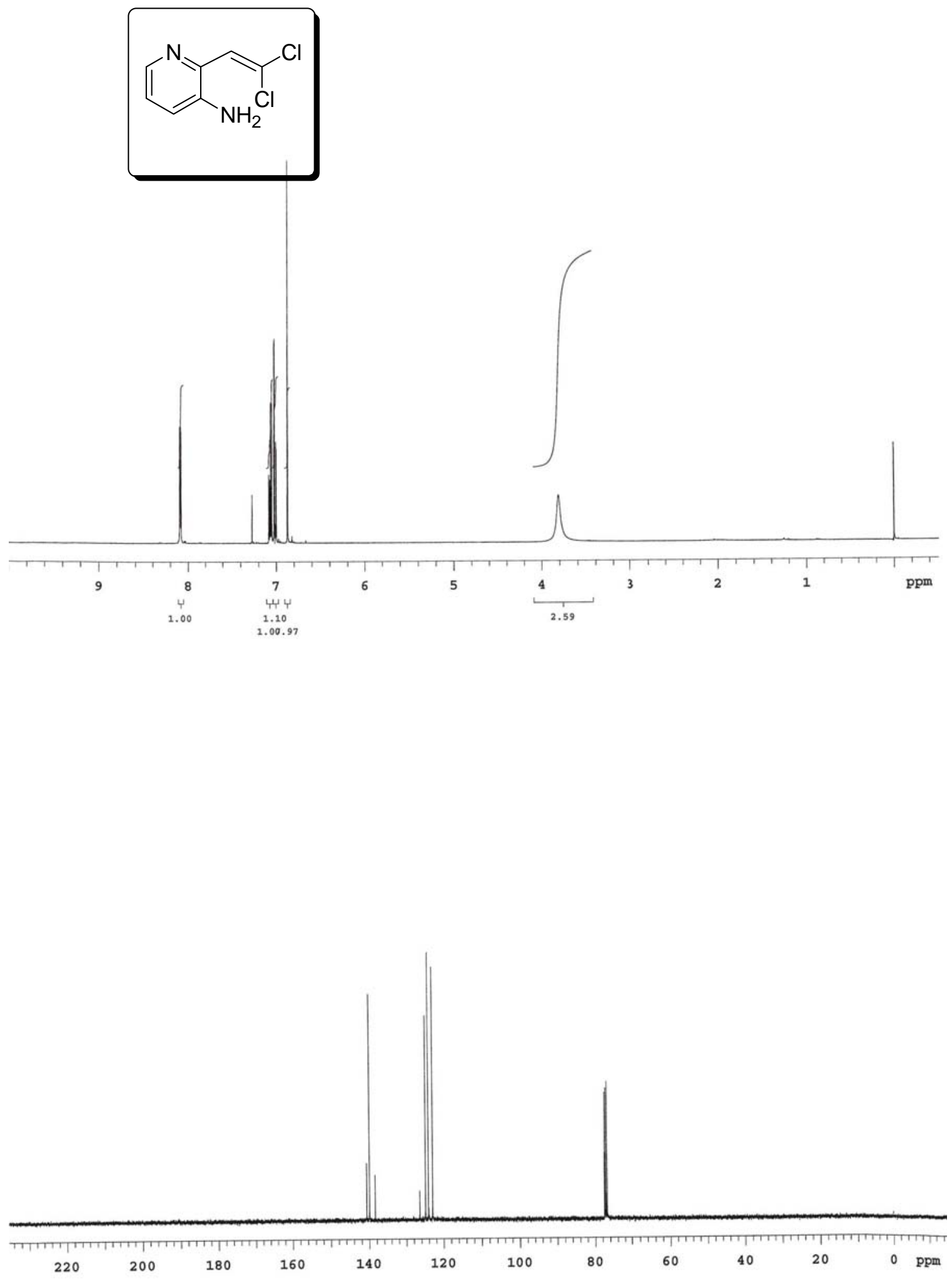
- S48 -
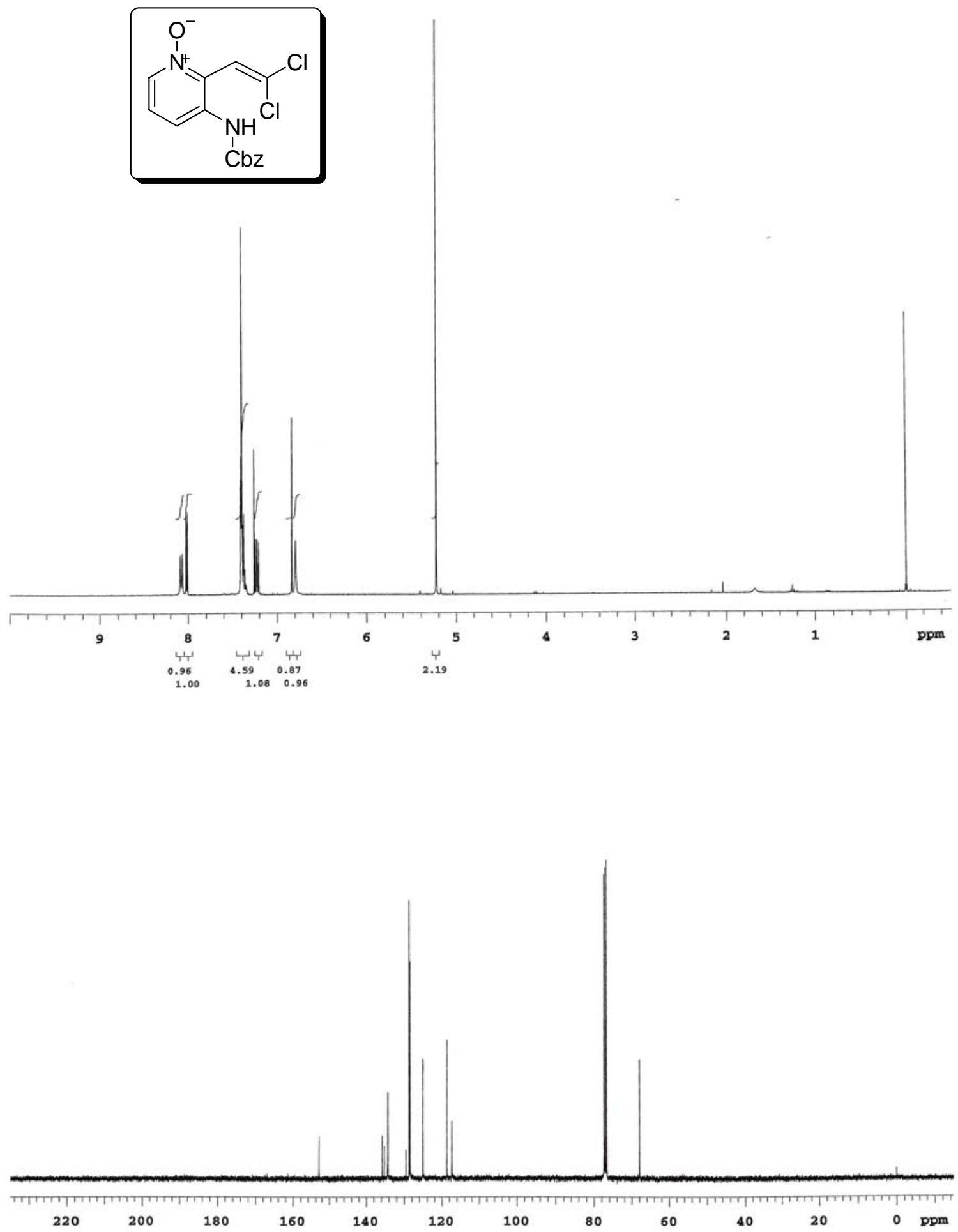
- S49 -
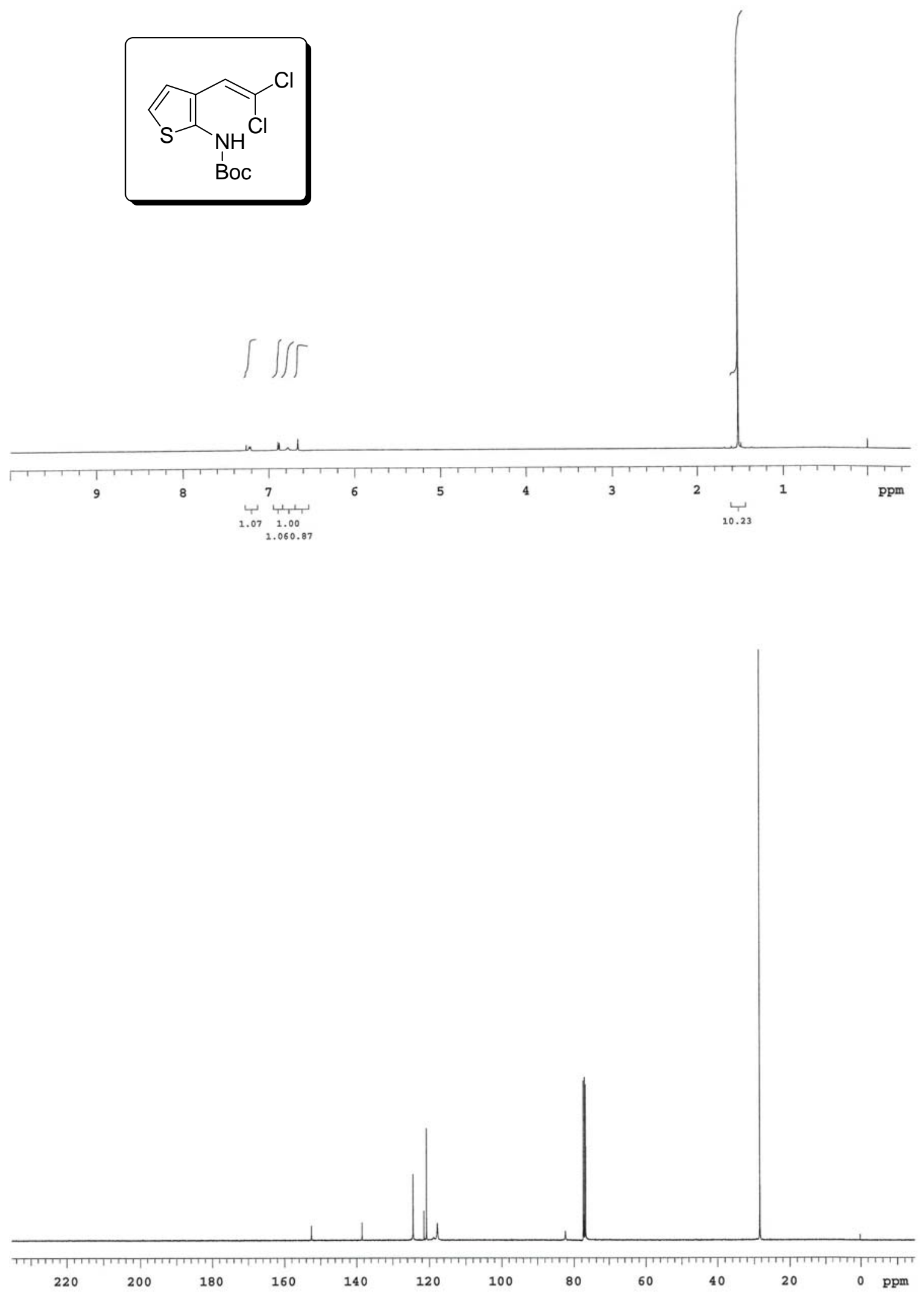
- S50 -

- S51 -
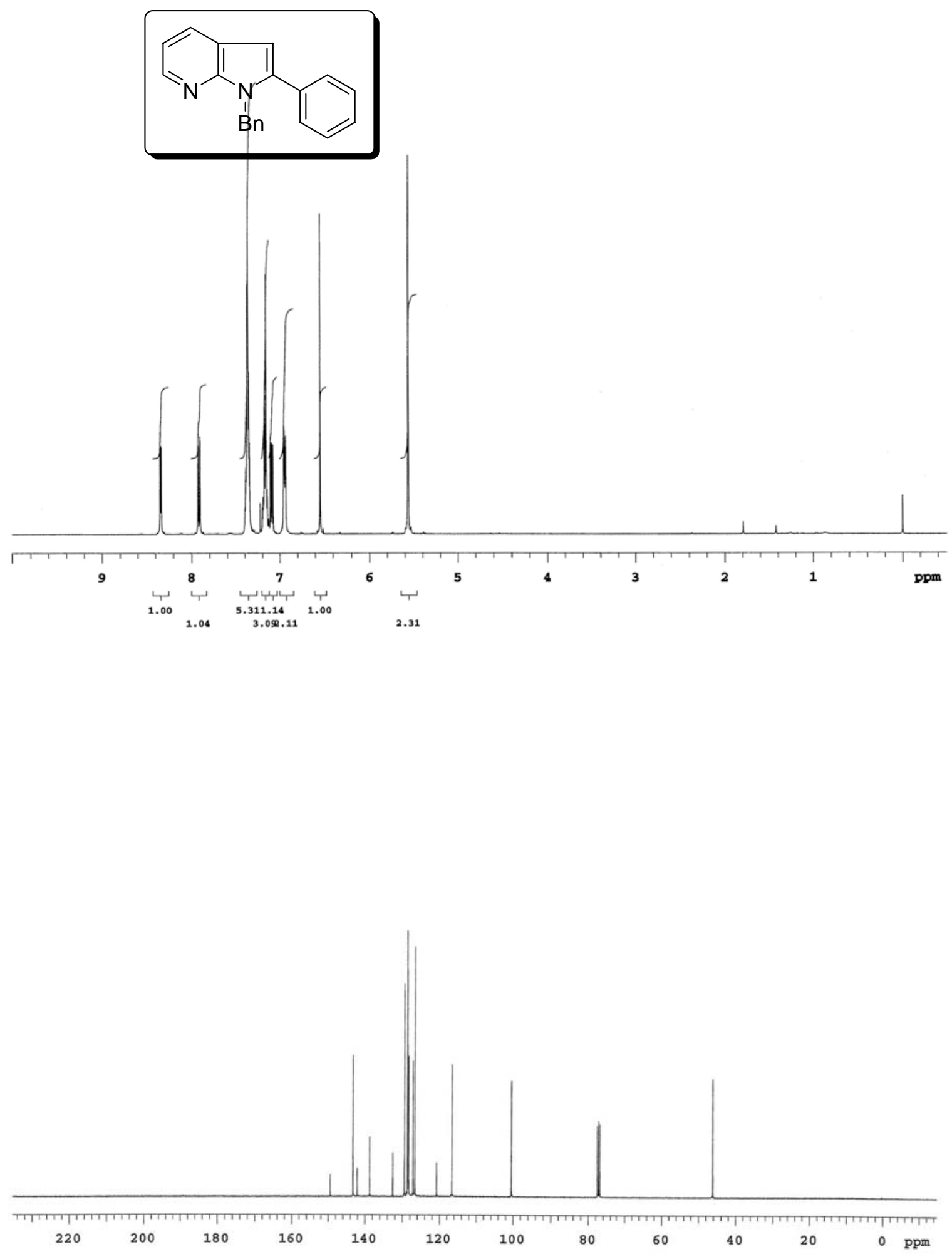

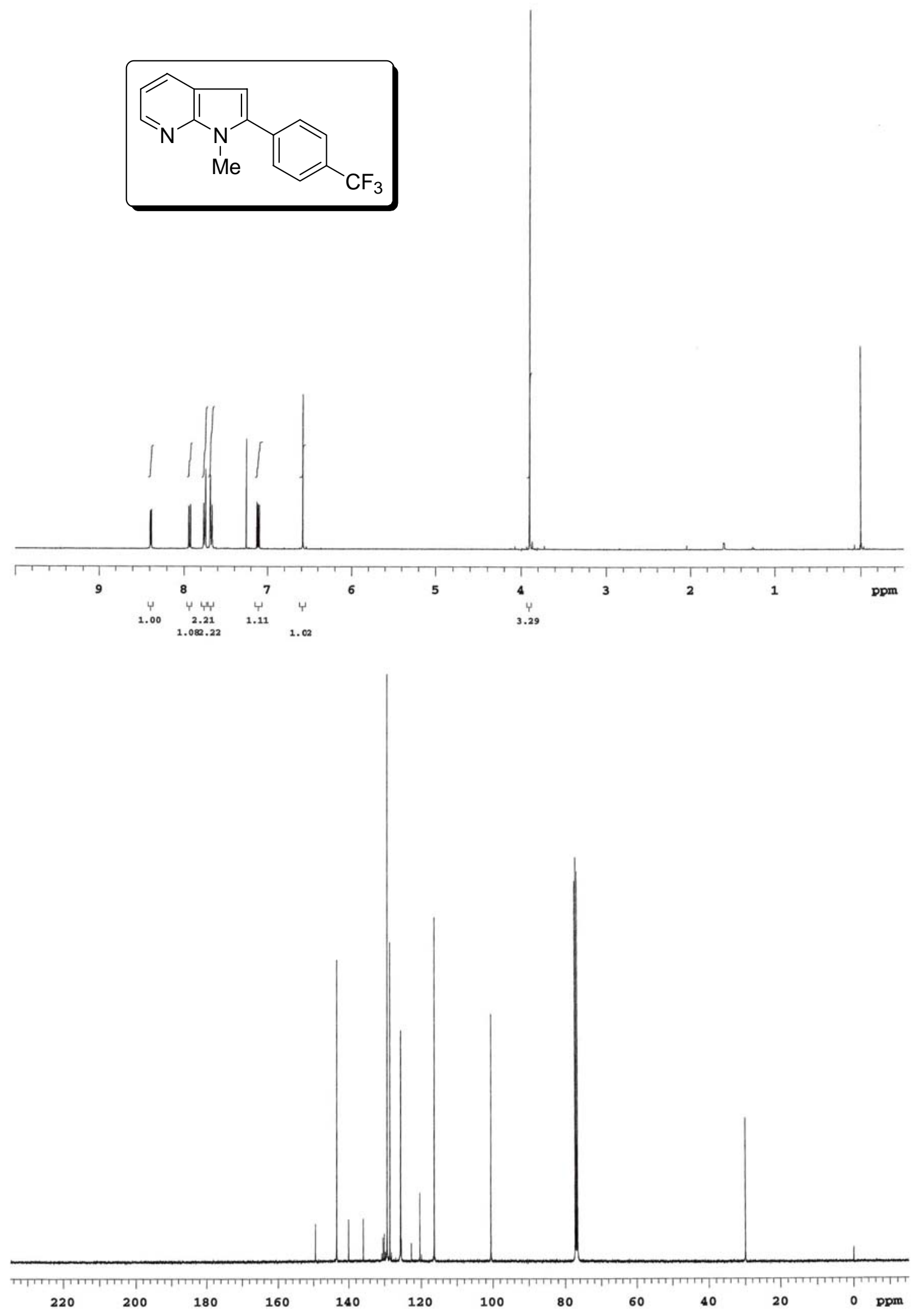
- S53 -
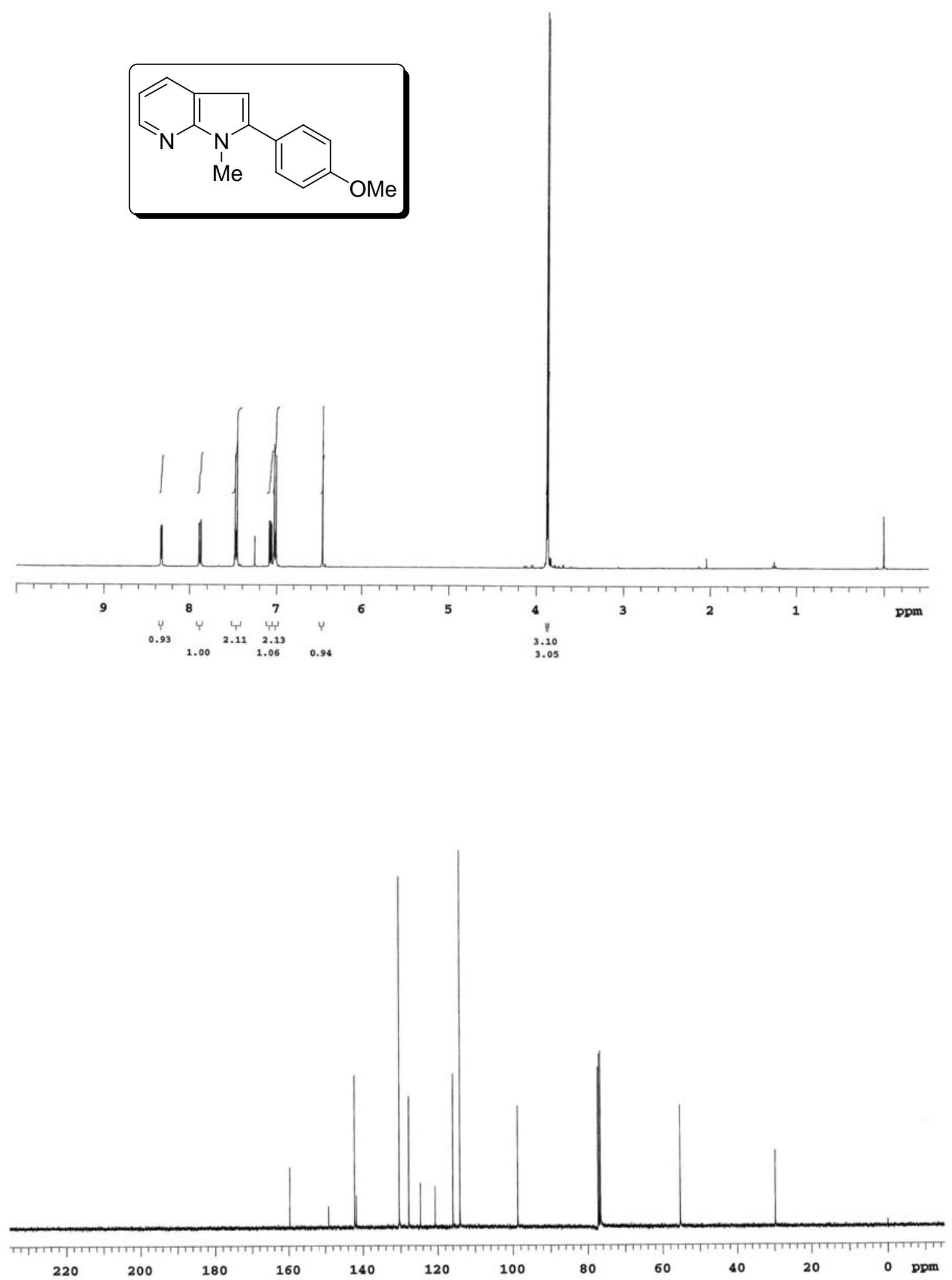
- S54 -

- S55 -
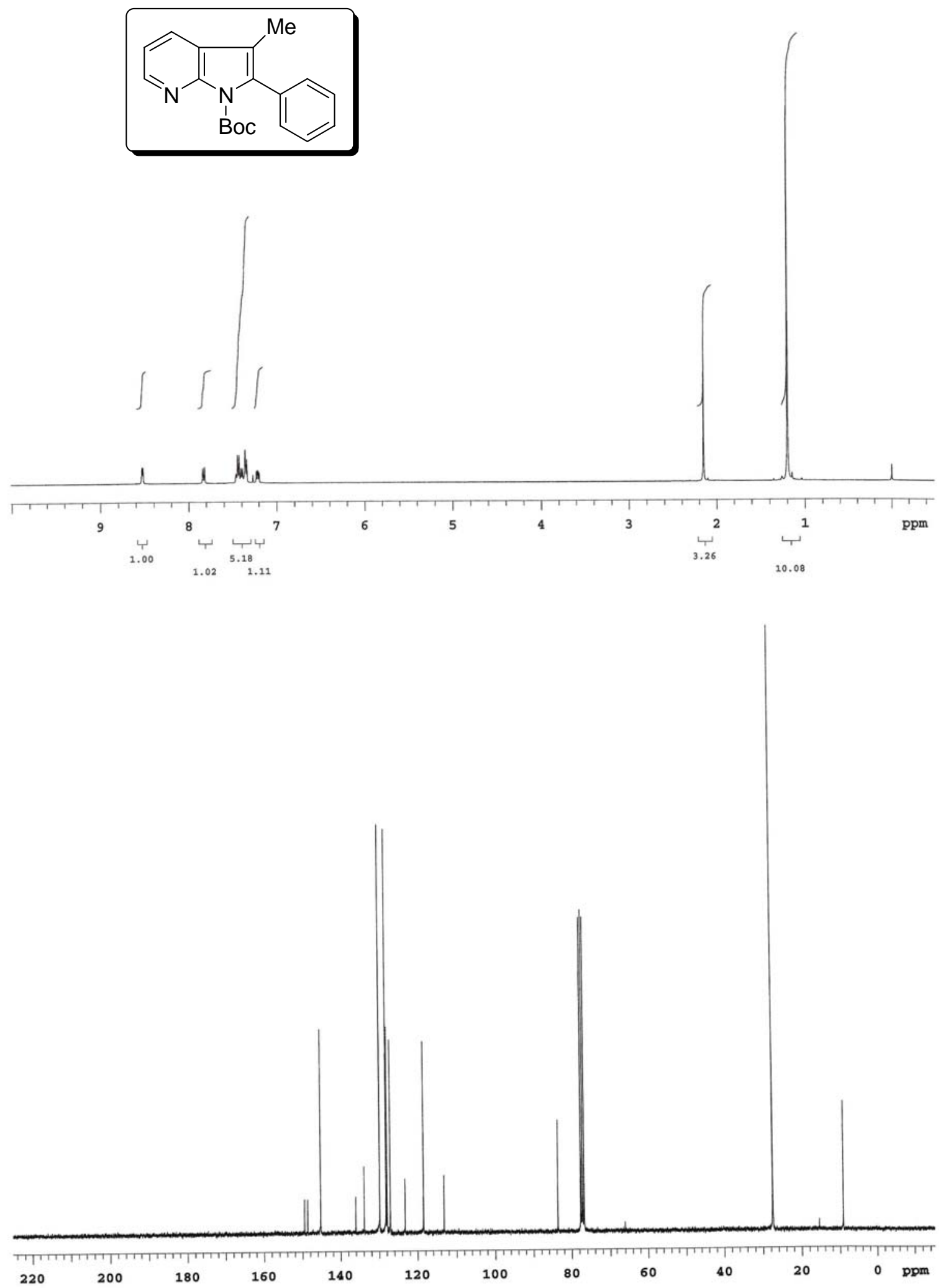
- S56 -
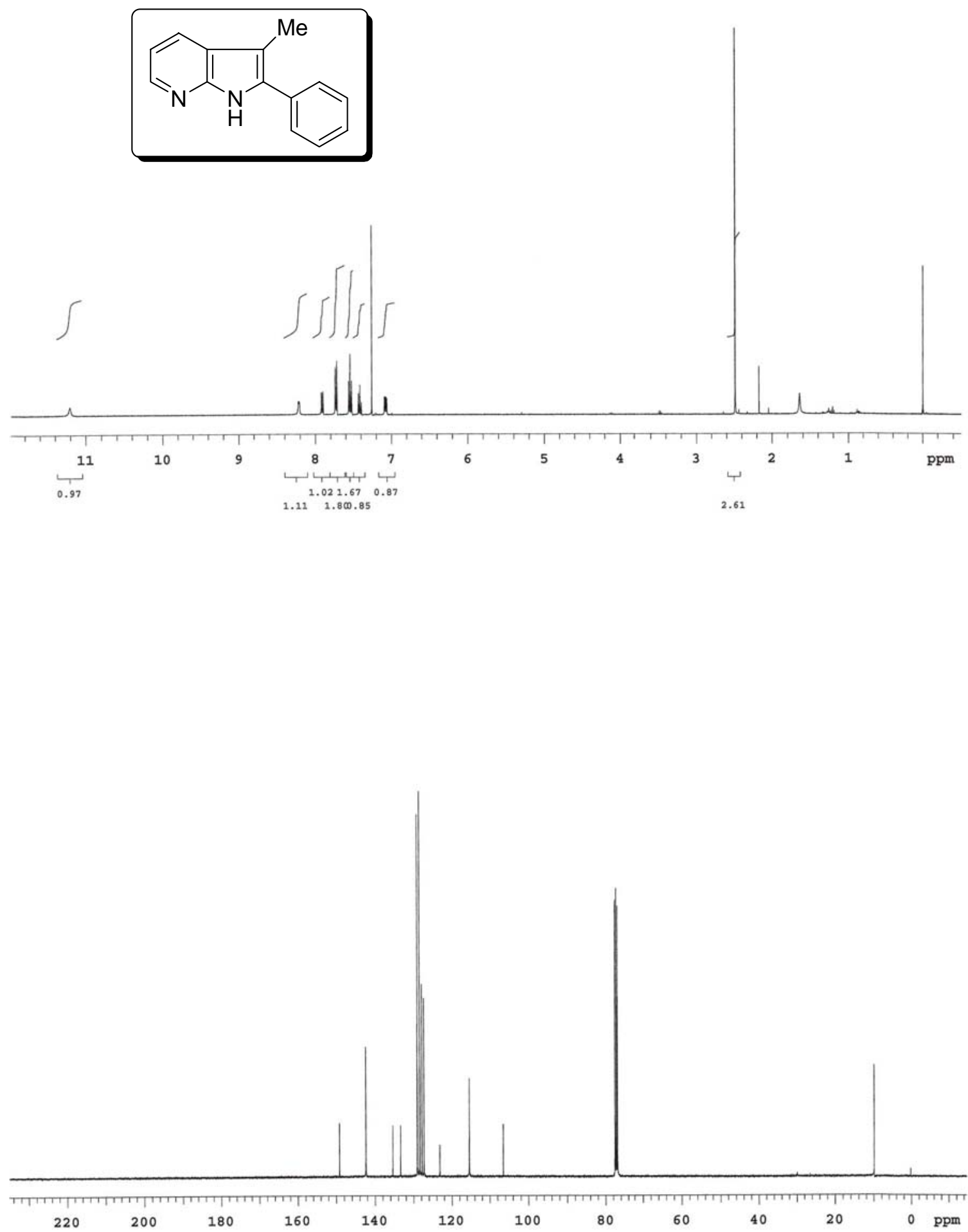
- S57 -

- S58 -
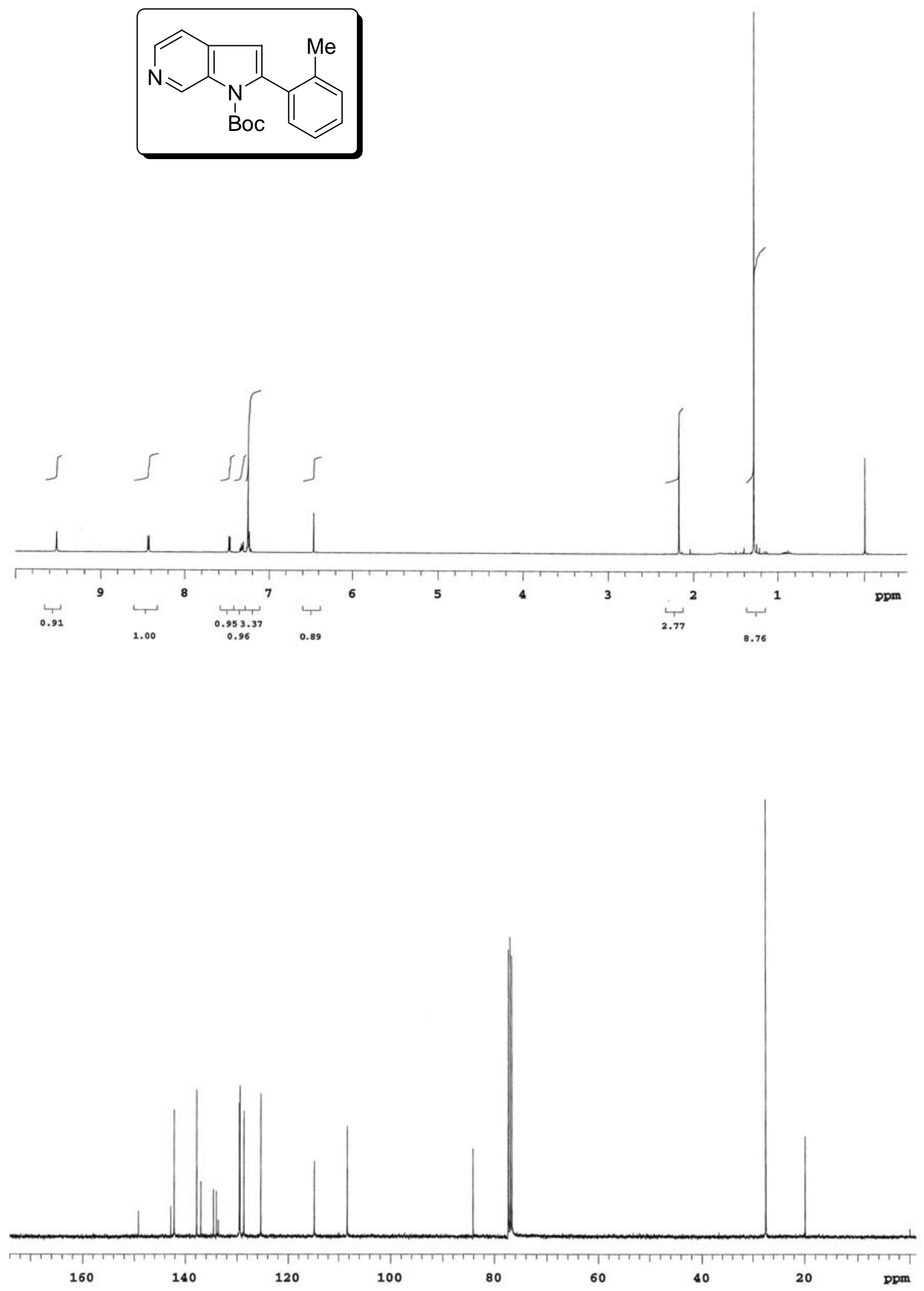

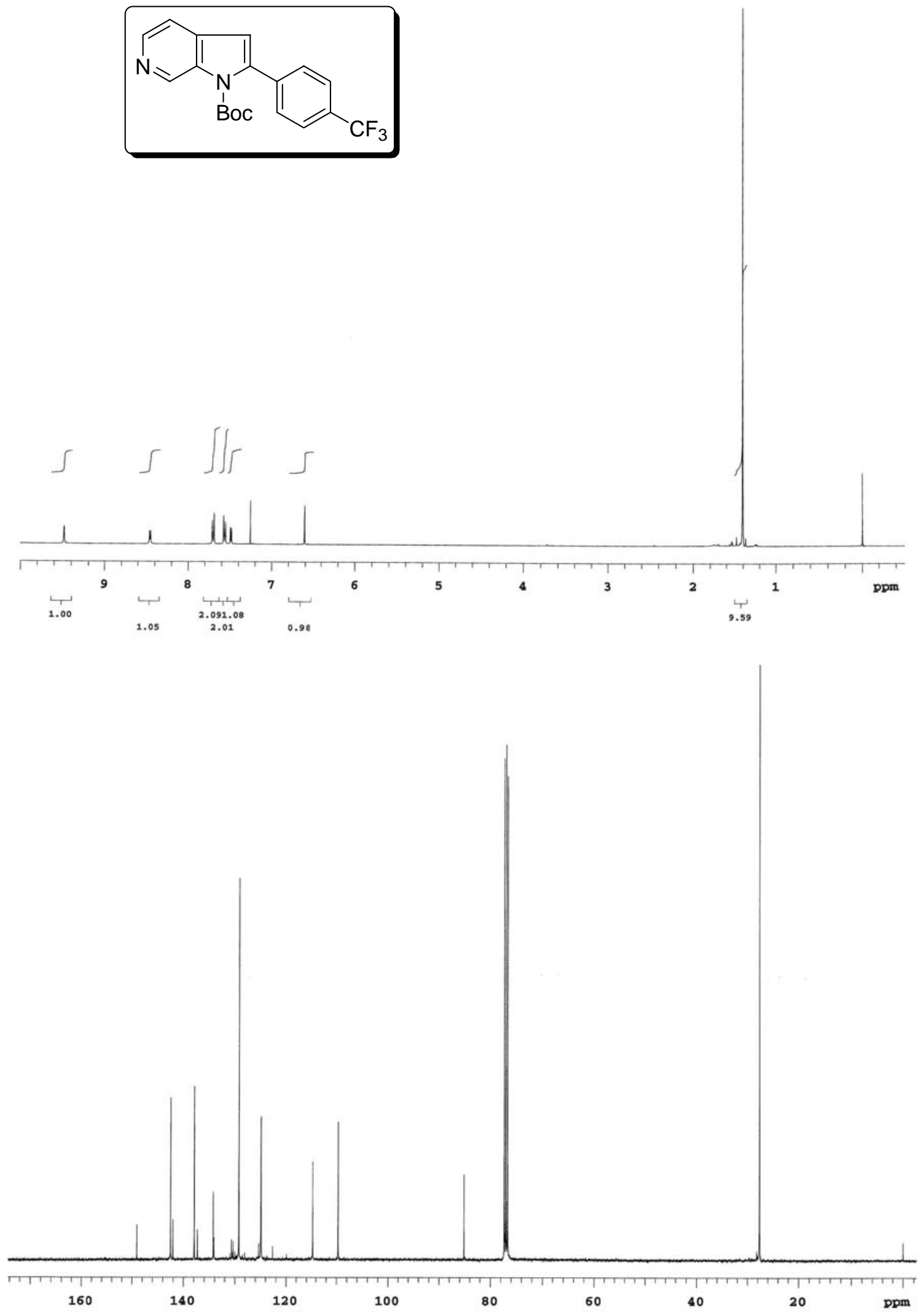
- S60 -

- S61 -
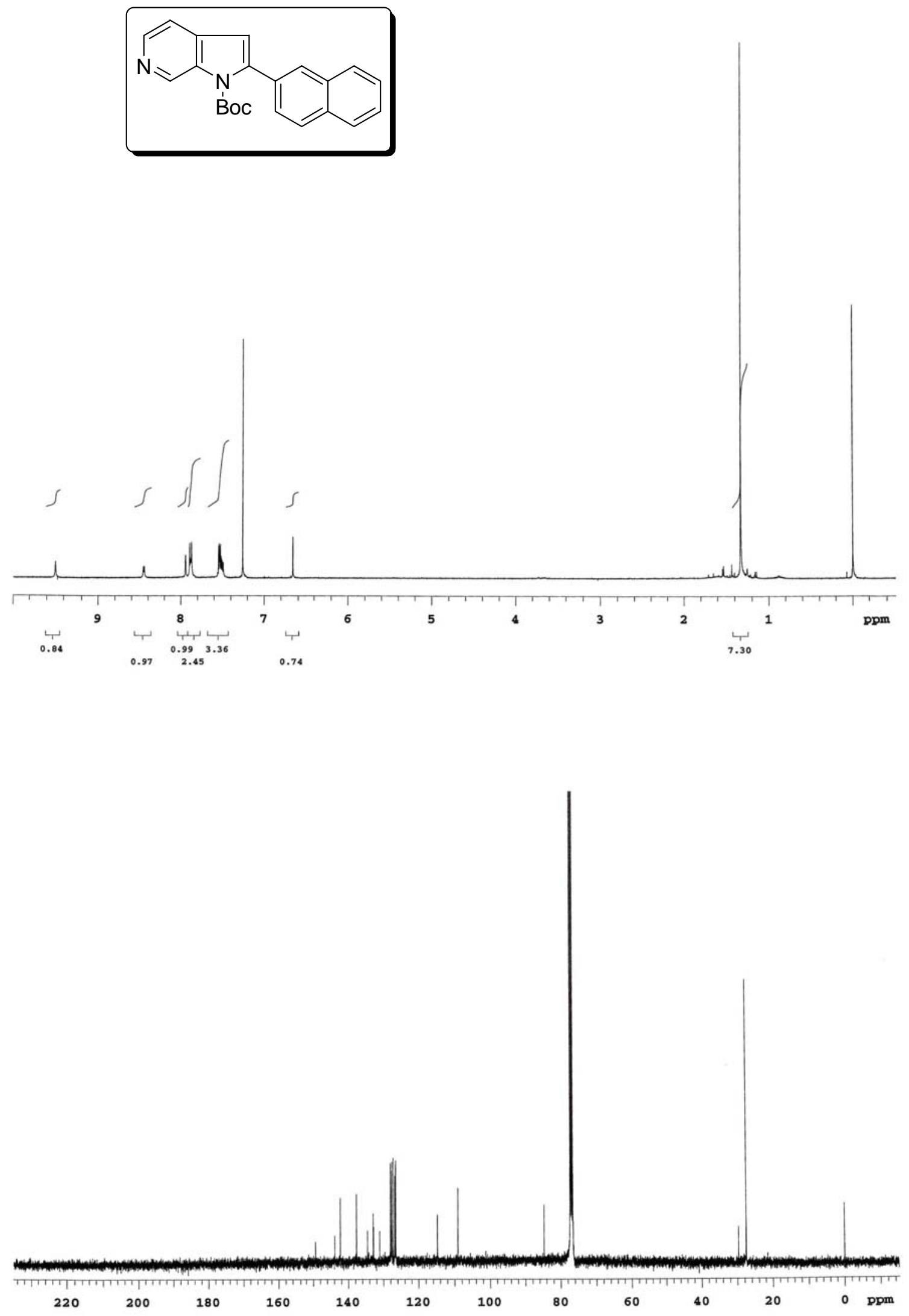
- S62 -

- S63 -
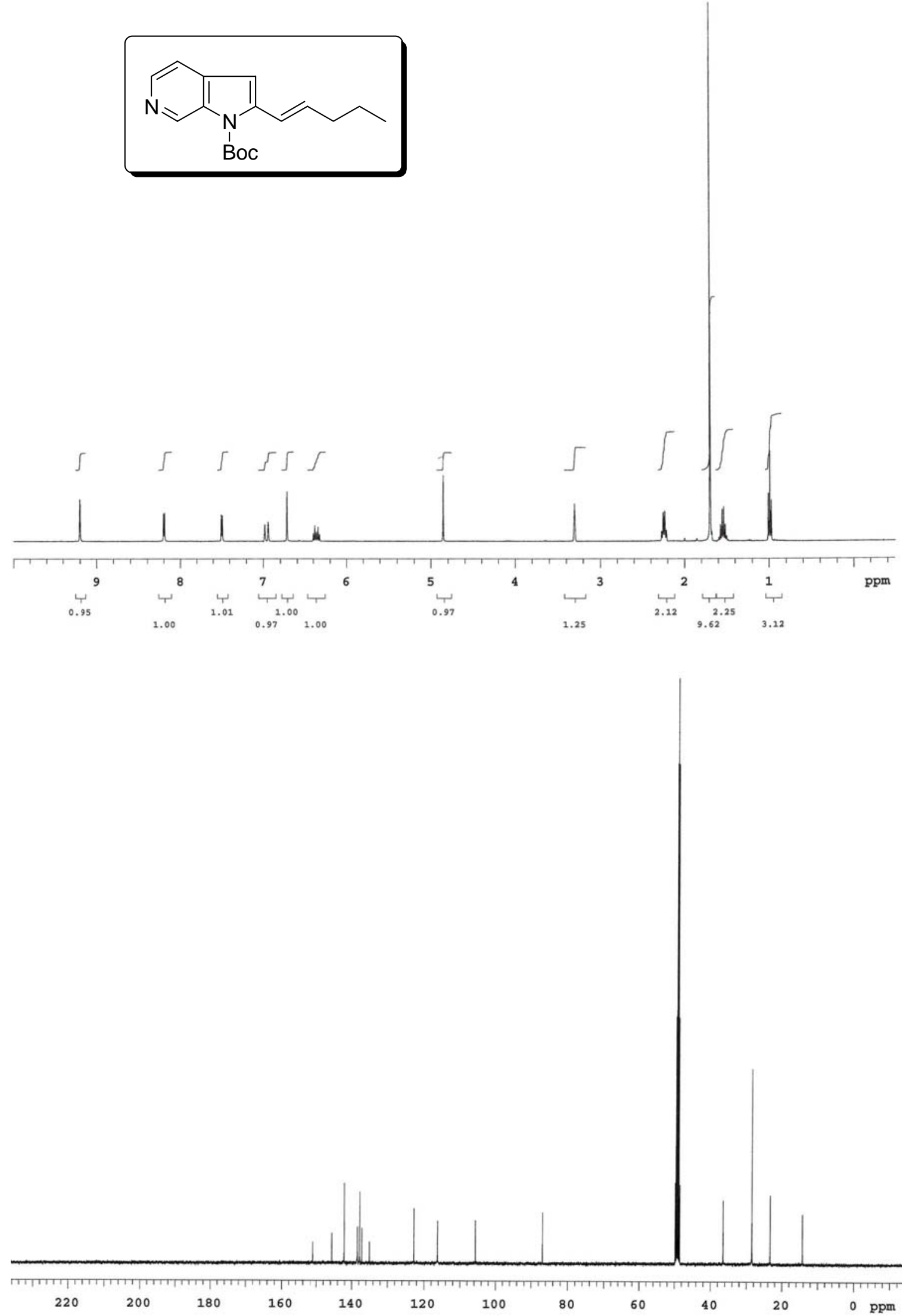
- S64 -
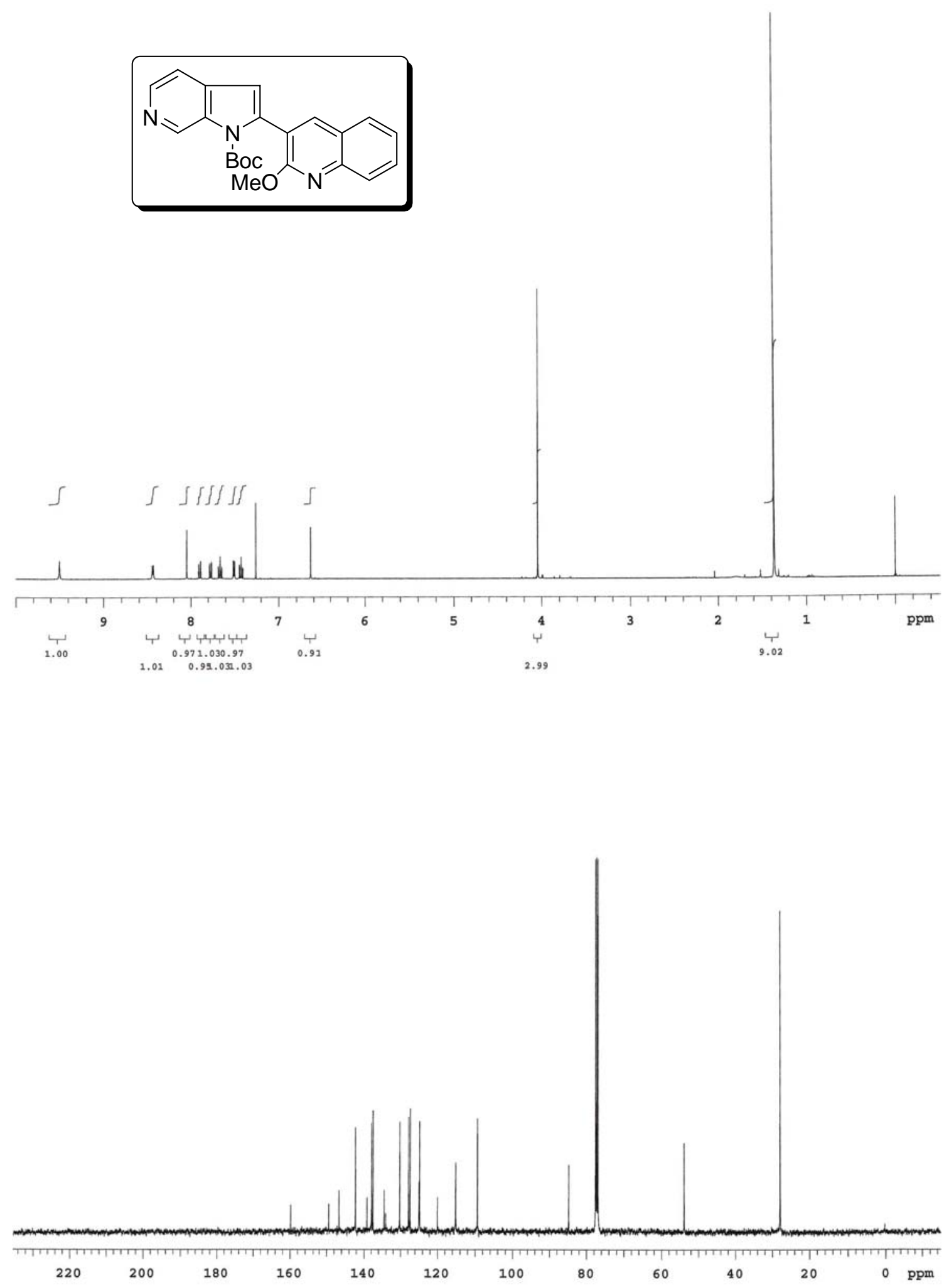
- S65 -
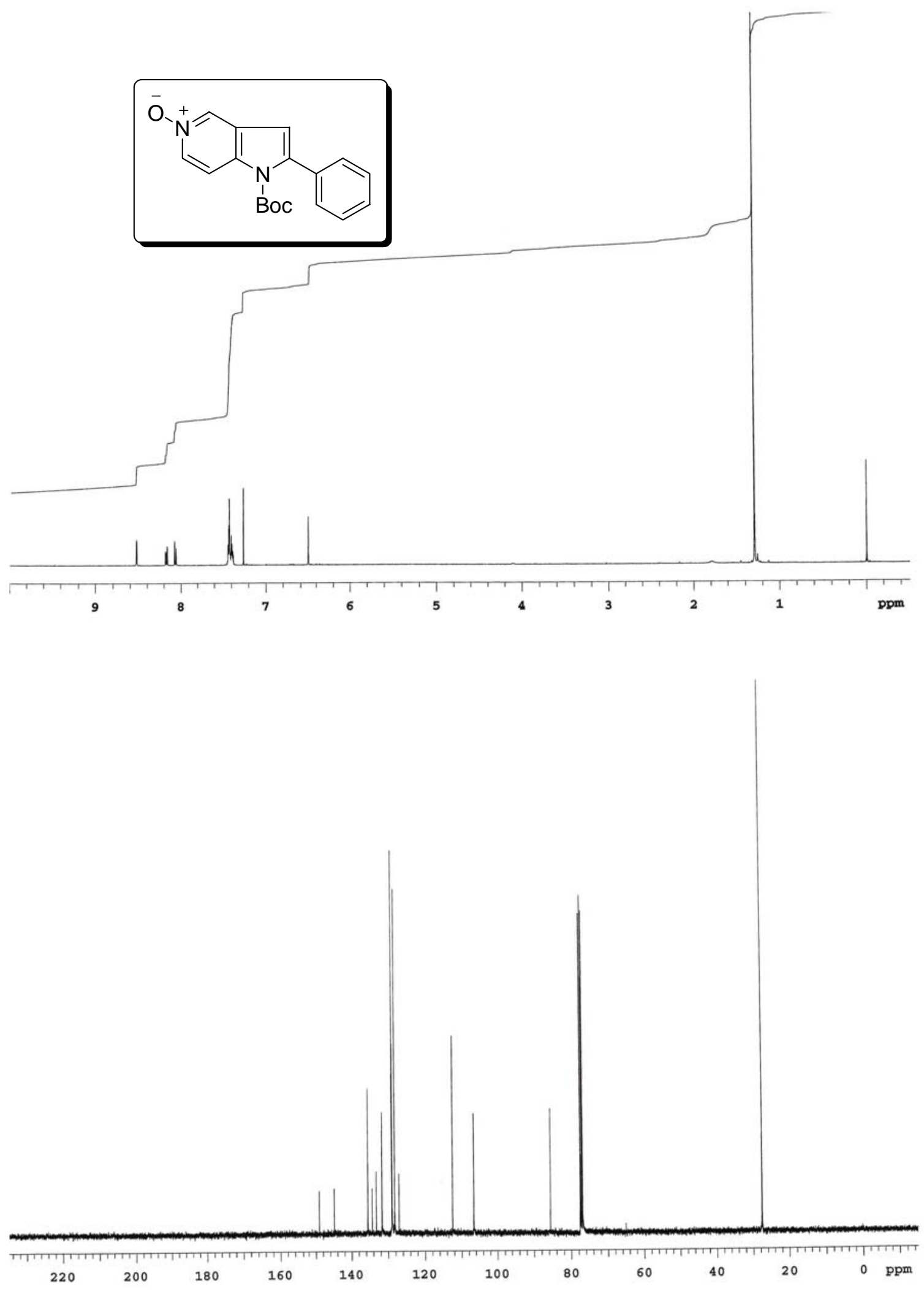
- S66 -
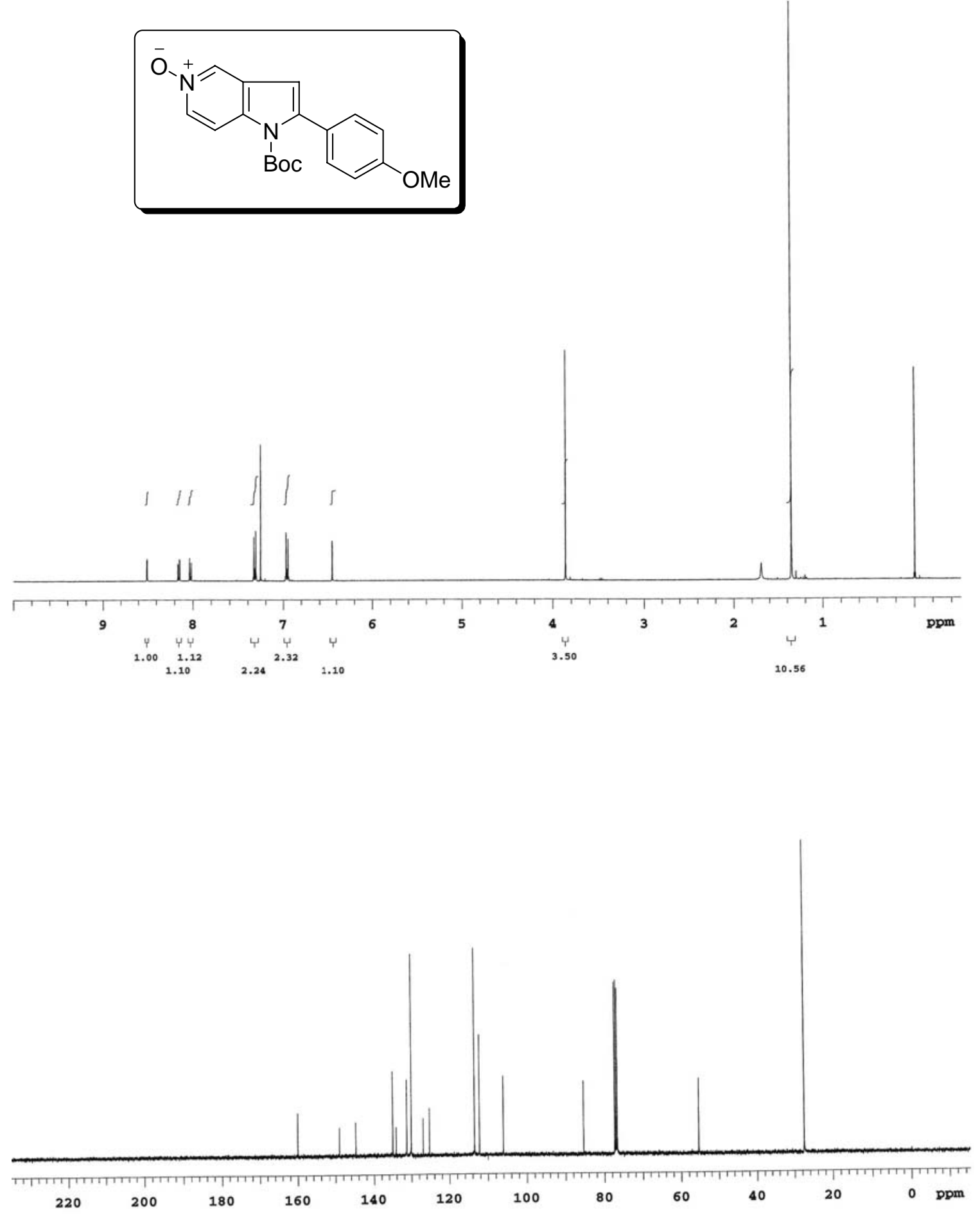
- S67 -
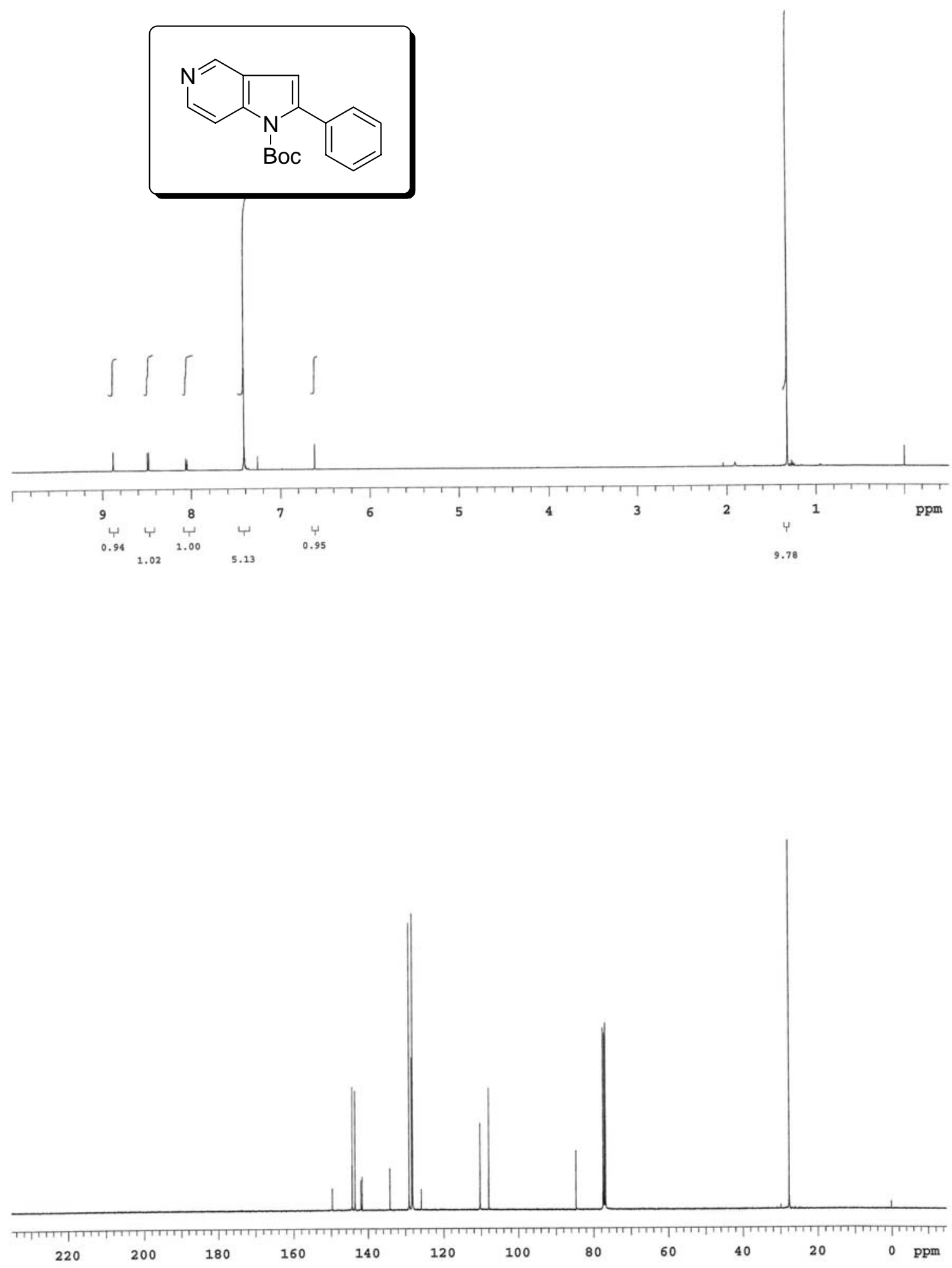
- S68 -

- S69 -
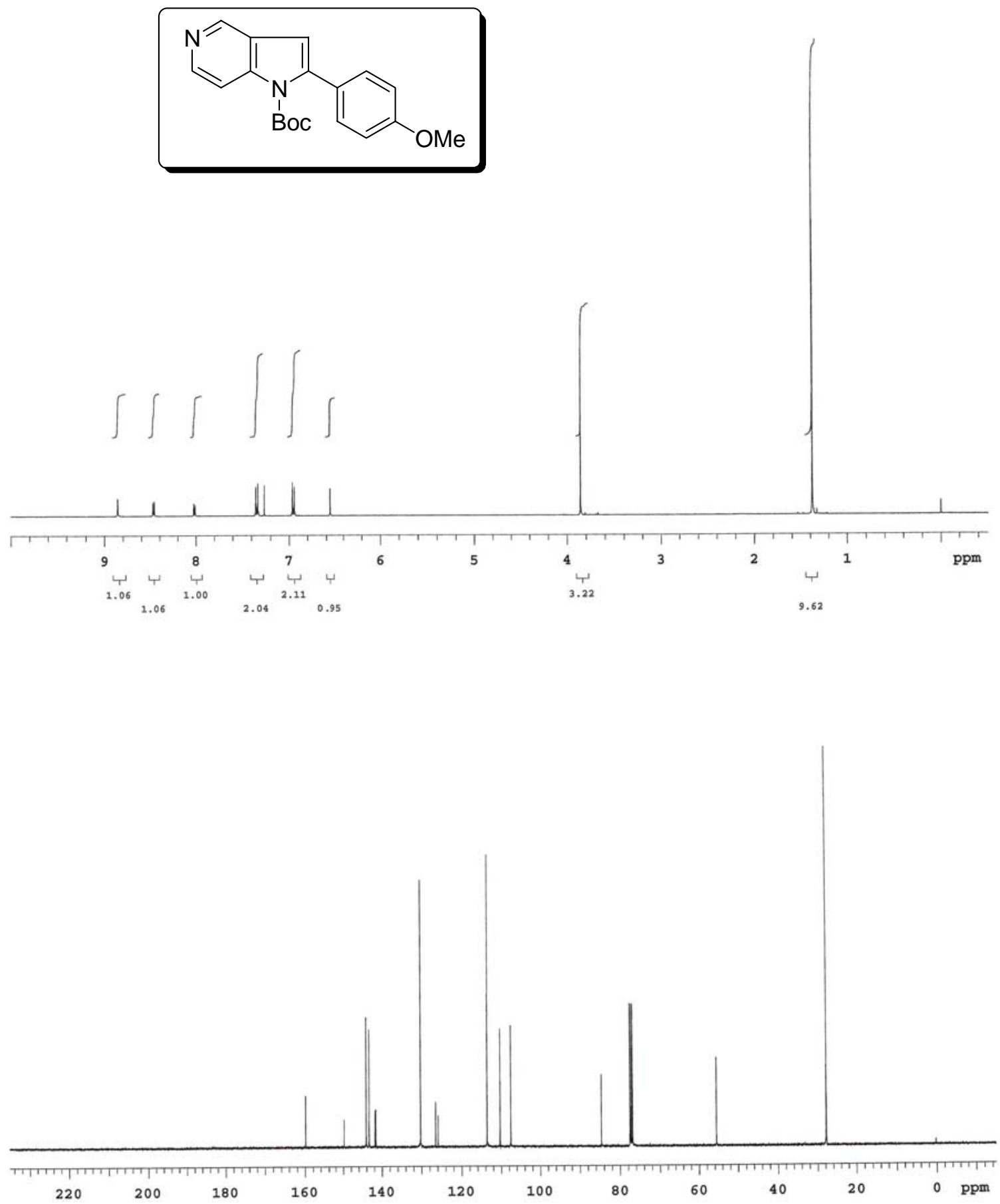



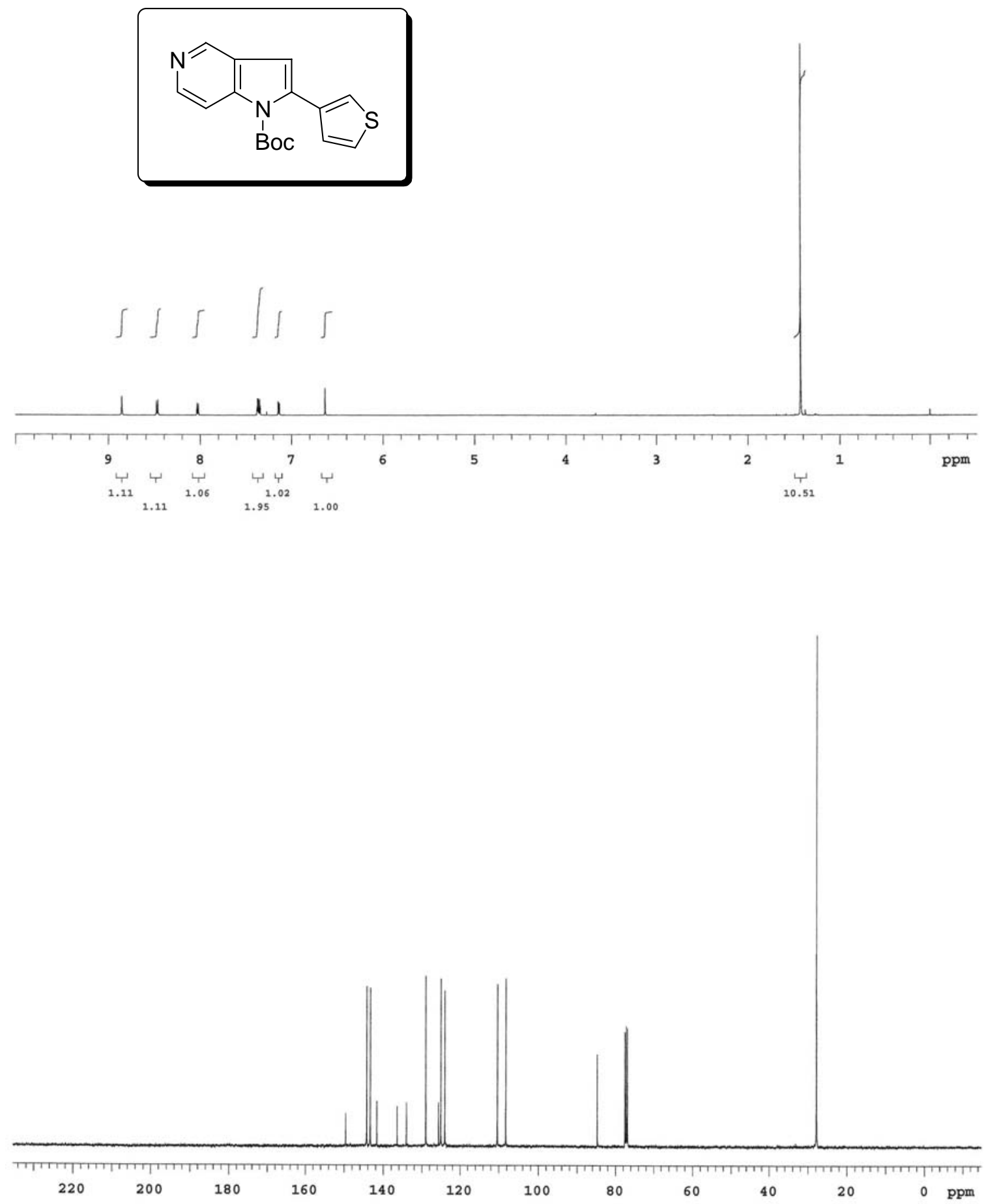

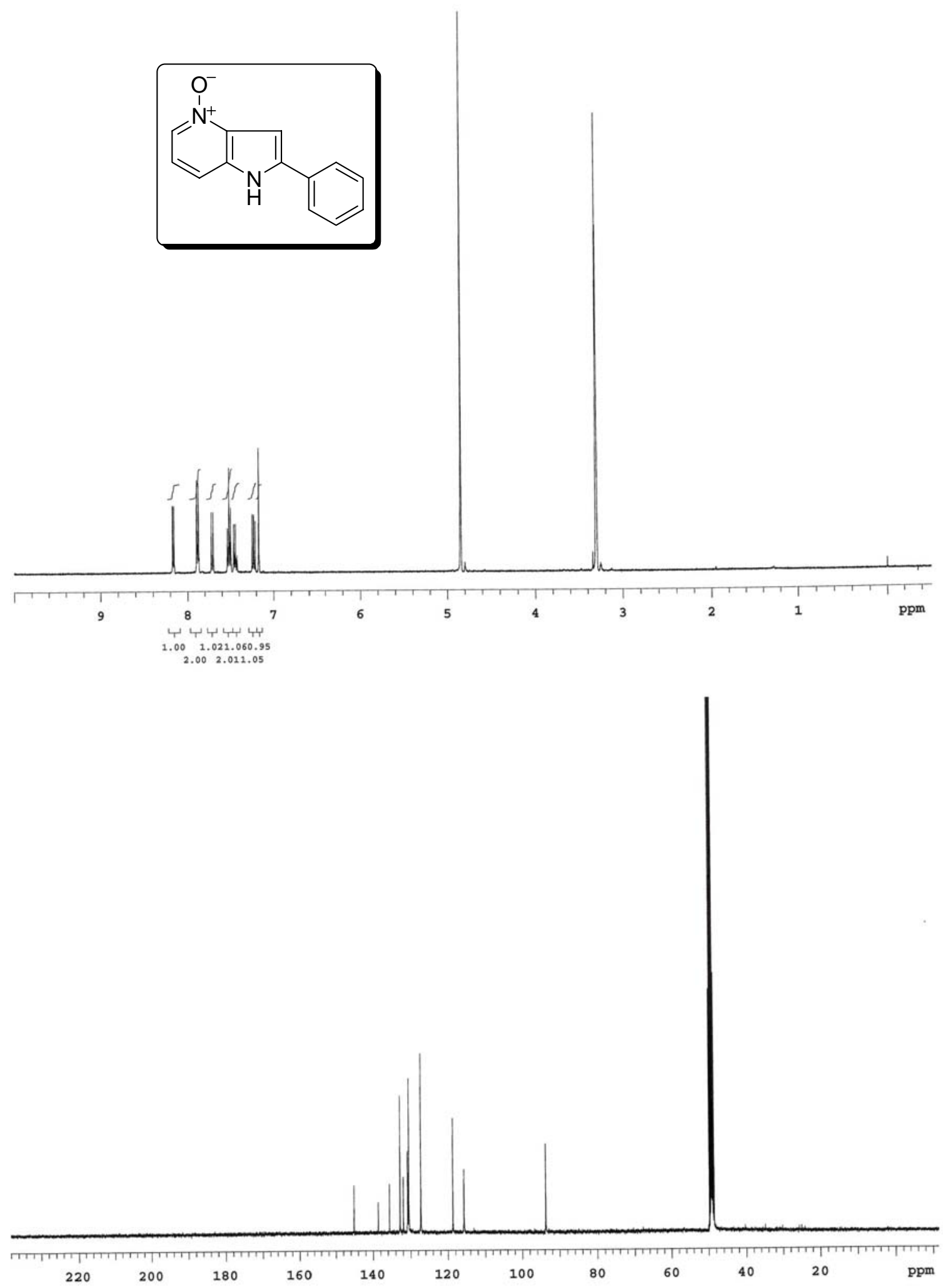
- S73 -




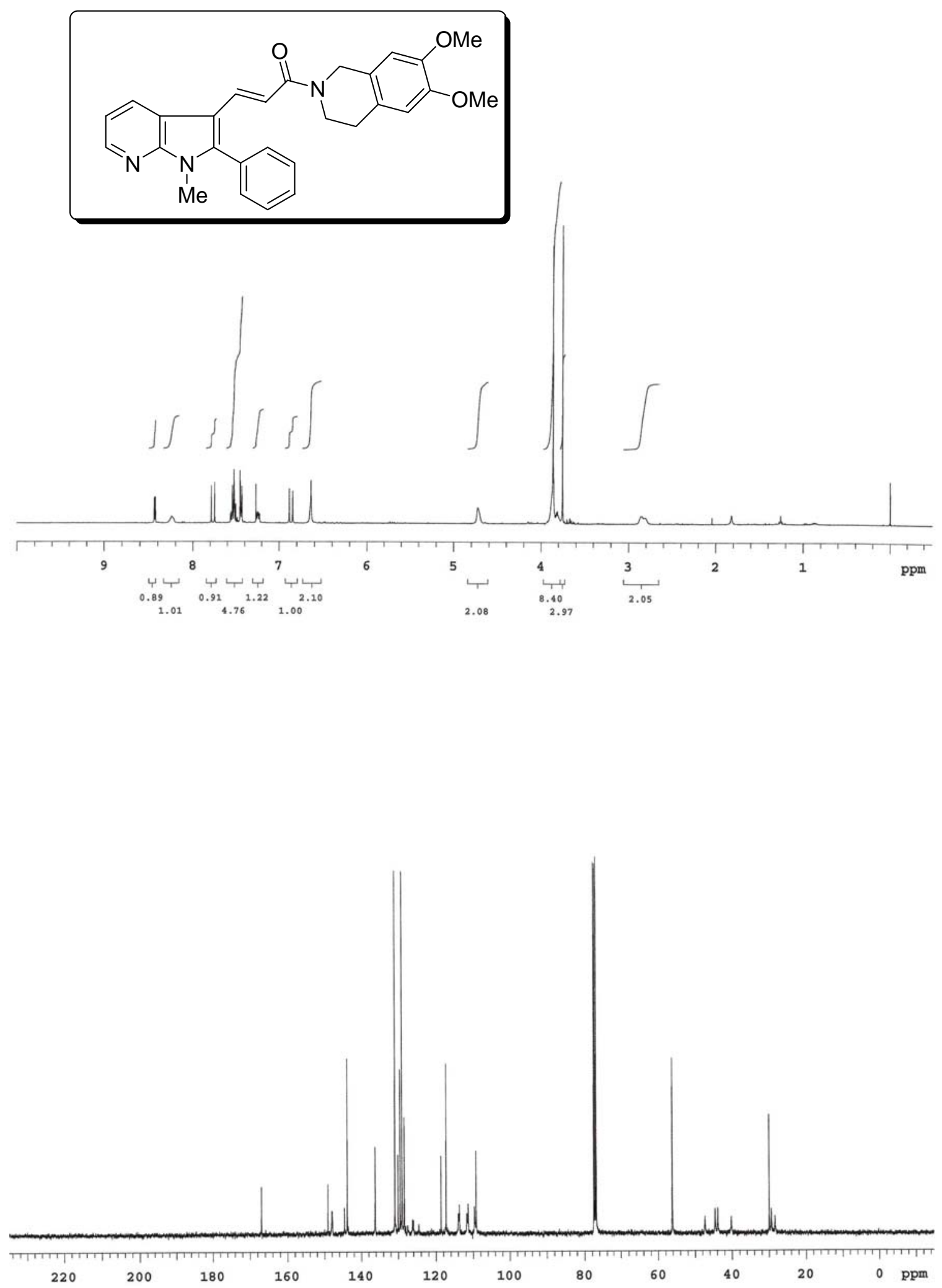


- S77 -
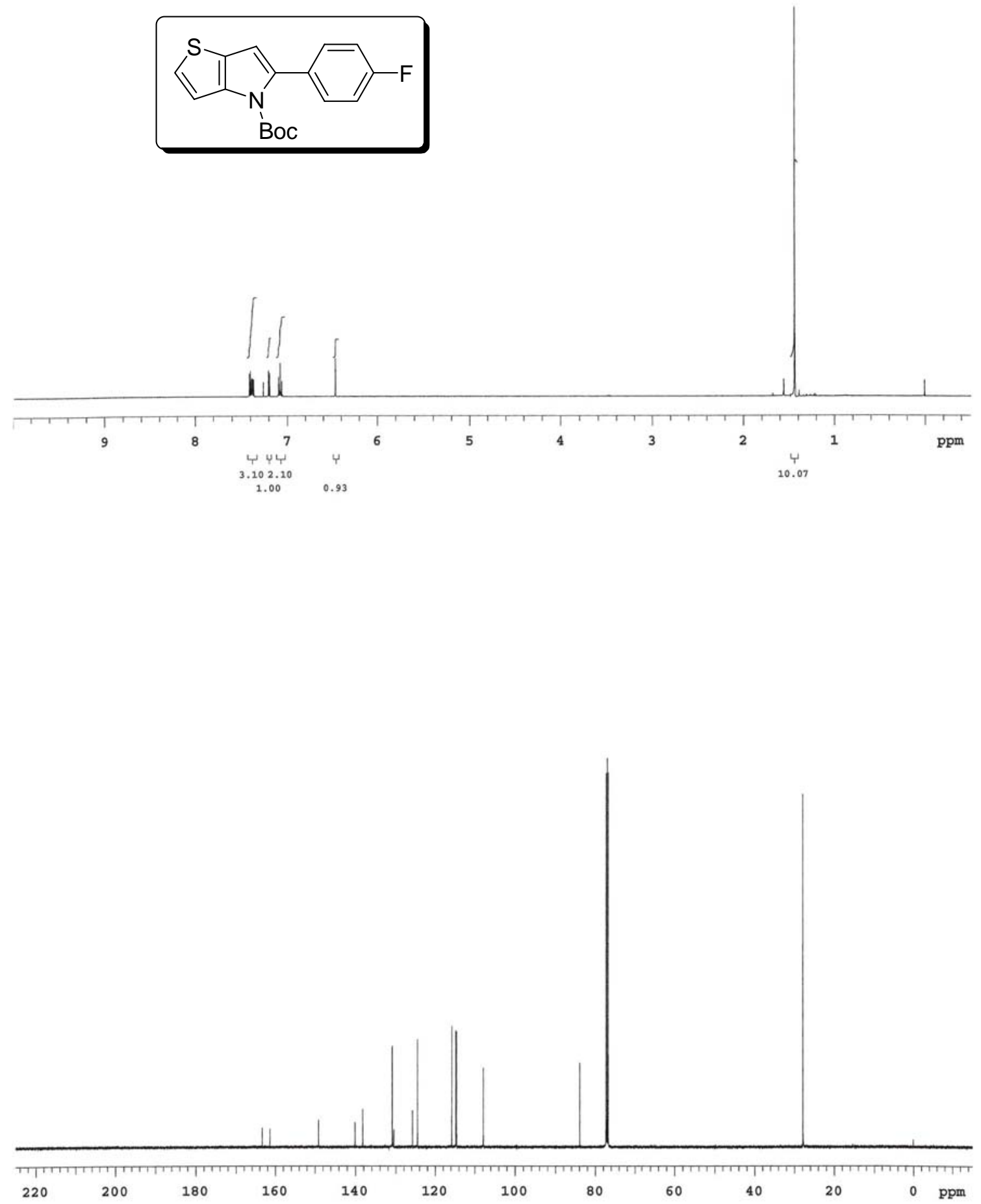
- S78 -



- S80 -
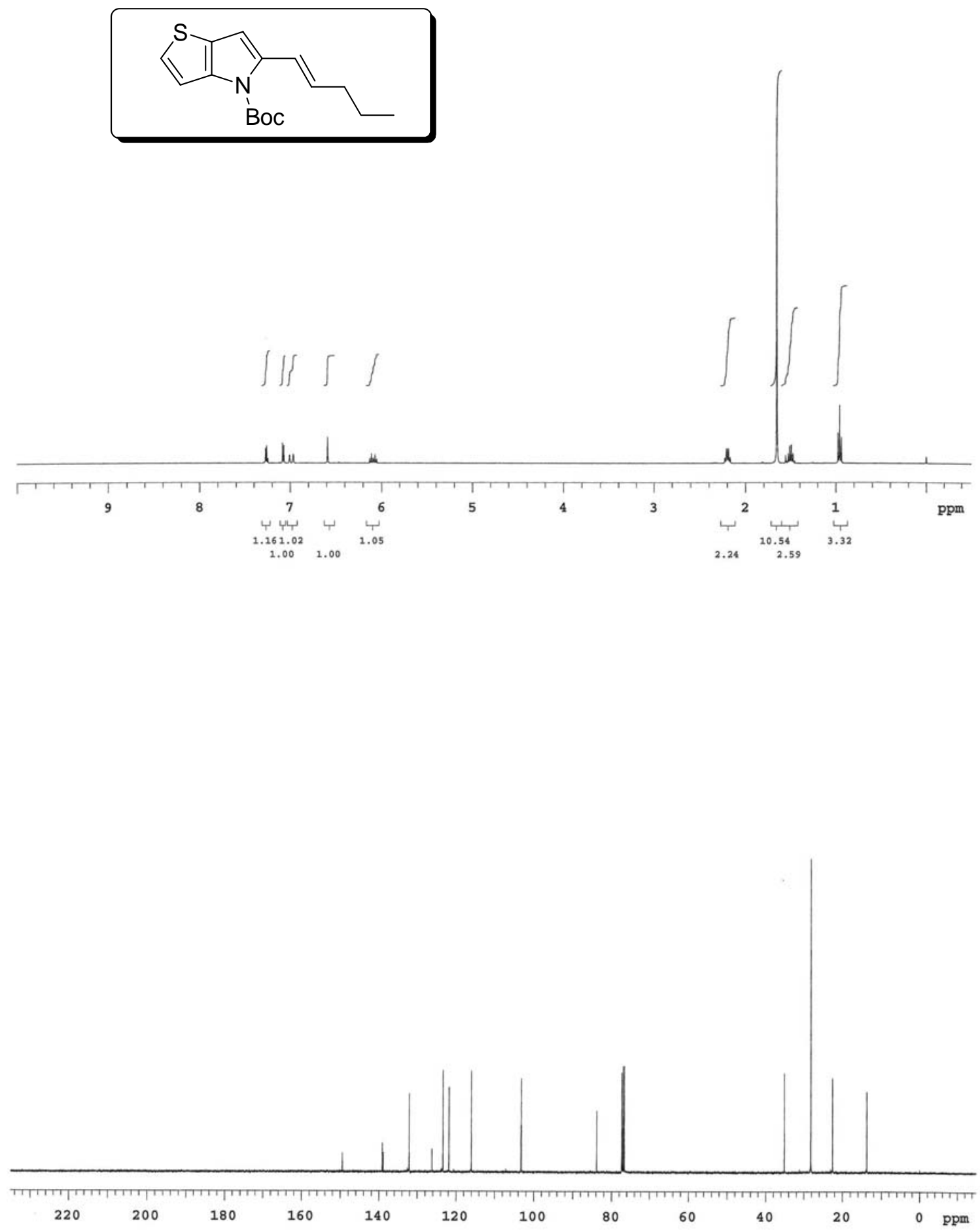
- S81 -
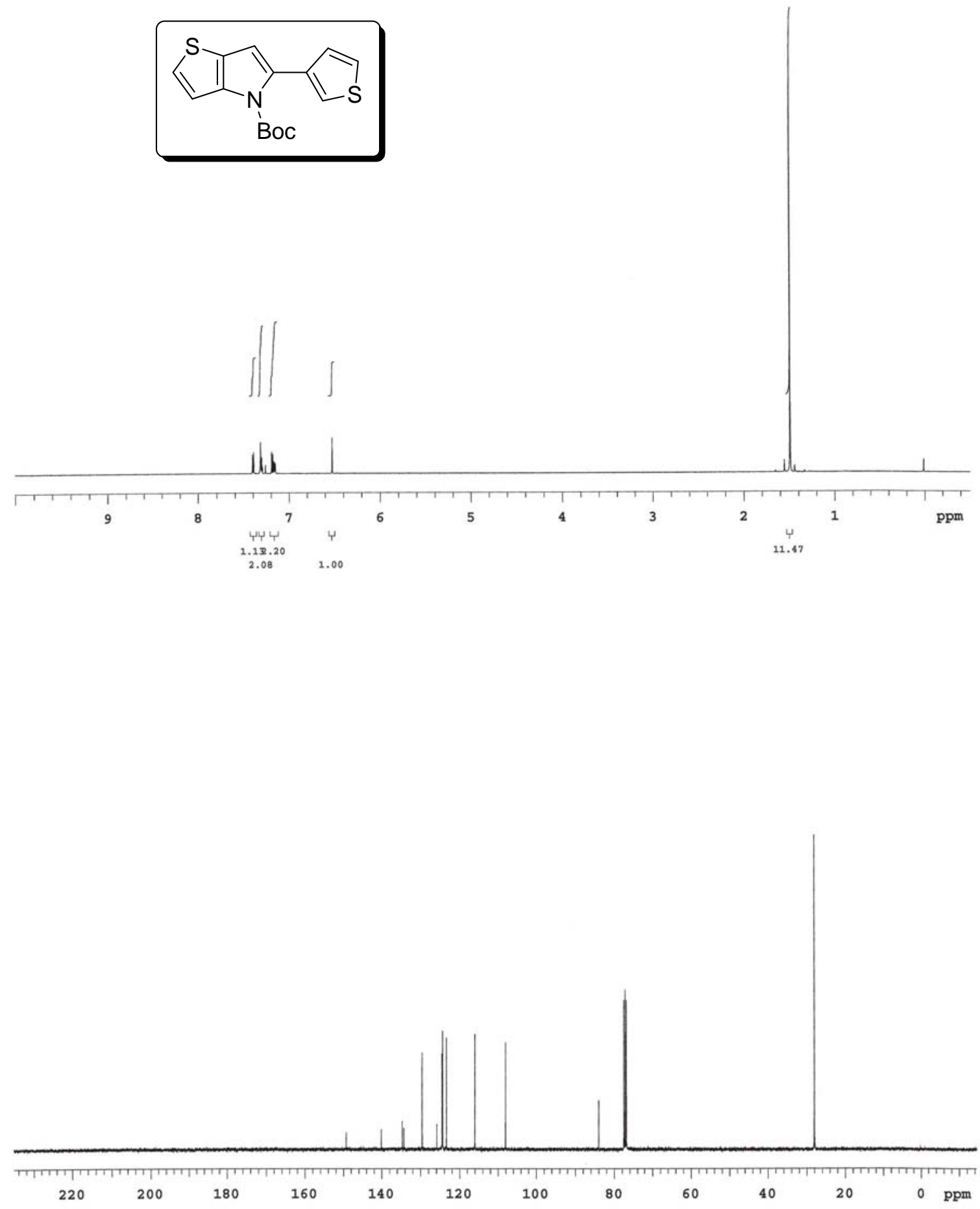
- S82 -

- S83 -
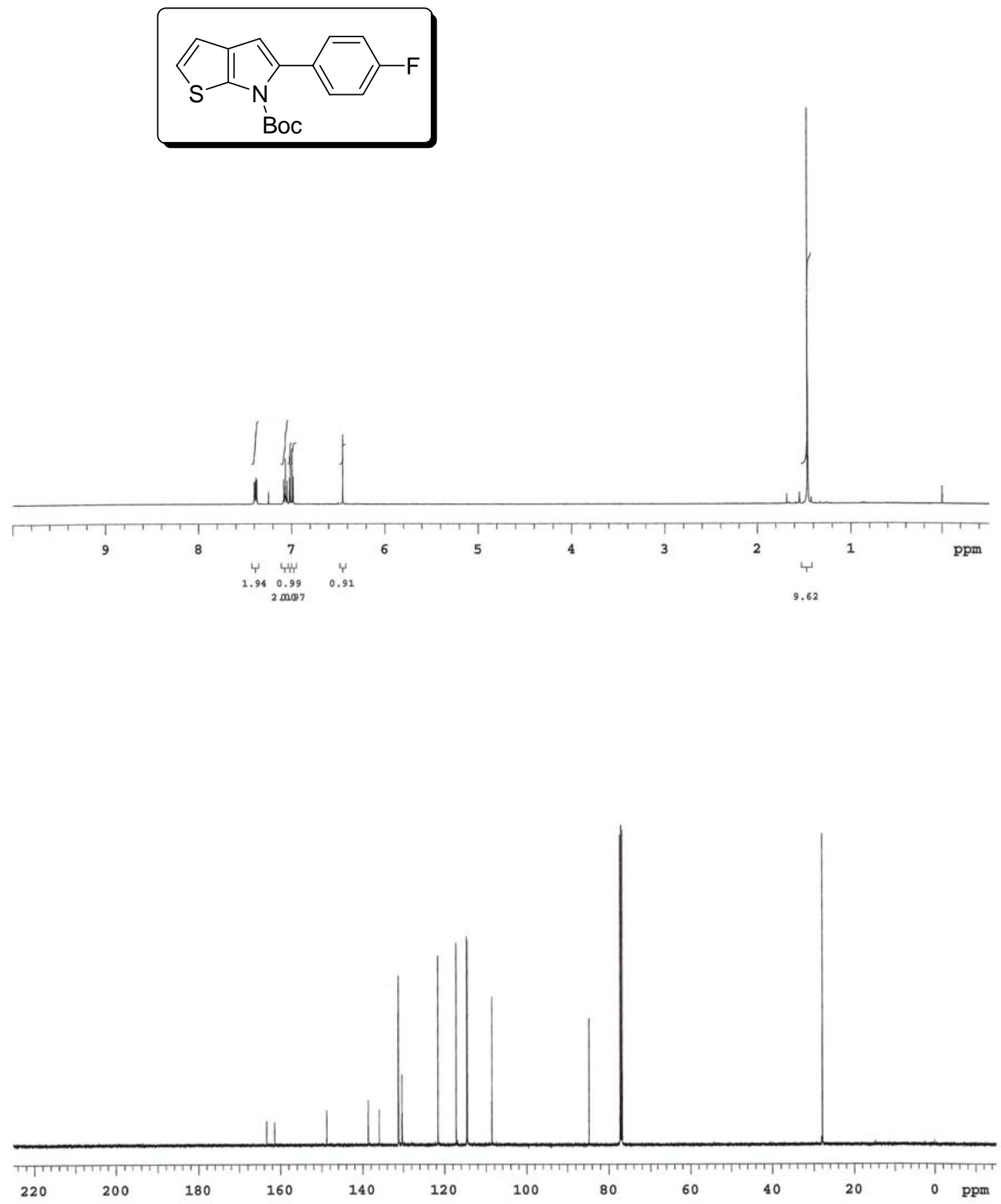
- S84 -
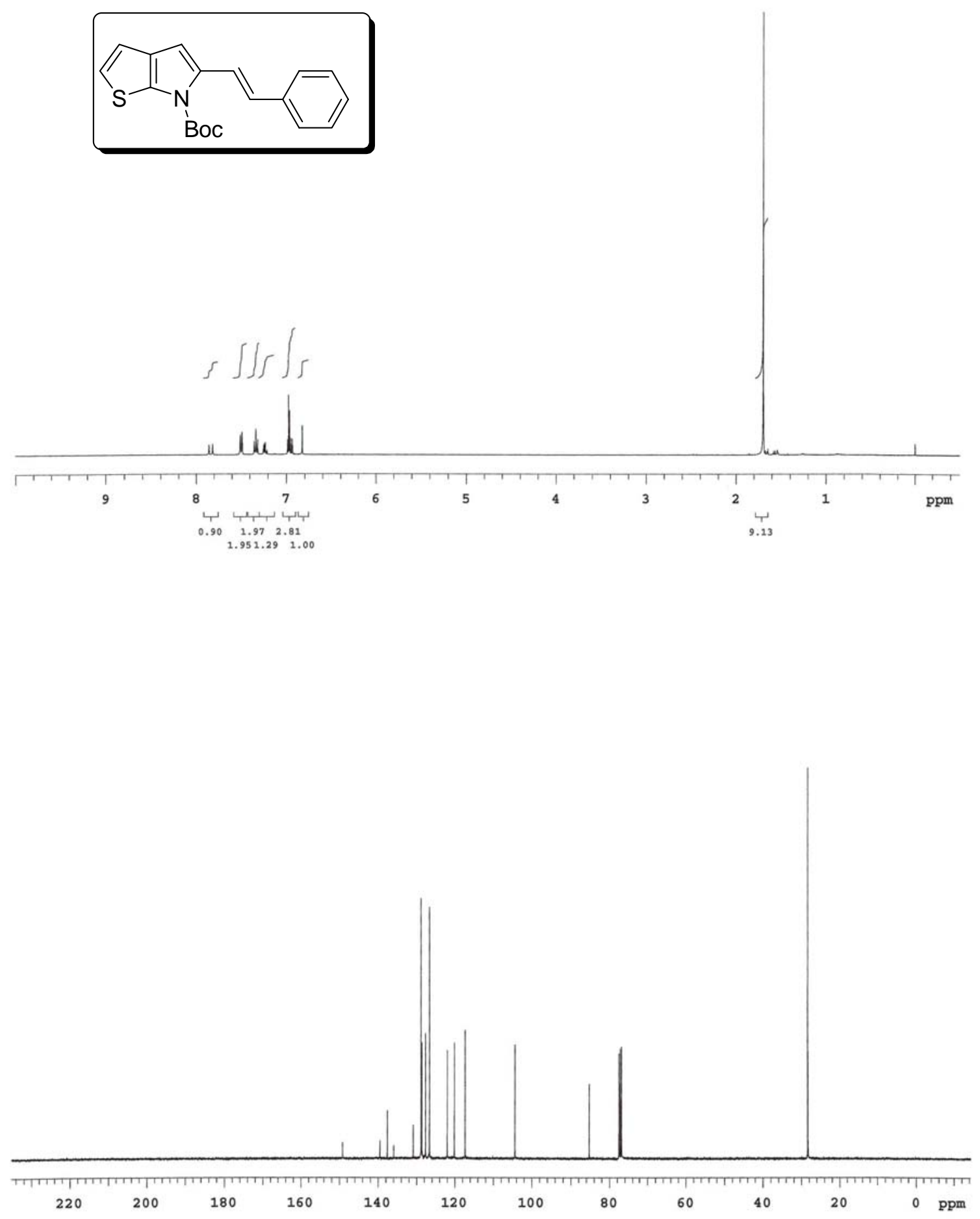
- S85 -

- S86 -
